TRANSACTIONS OF THE

AMERICAN MATHEMATICAL SOCIETY

Volume 358, Number 10, October 2006, Pages 4395-4439

S 0002-9947(06)04190-0

Article electronically published on May 9, 2006

\title{
A PREPARATION THEOREM FOR WEIERSTRASS SYSTEMS
}

\author{
DANIEL J. MILLER
}

\begin{abstract}
It is shown that Lion and Rolin's preparation theorem for globally subanalytic functions holds for the collection of definable functions in any expansion of the real ordered field by a Weierstrass system.
\end{abstract}

\section{INTRODUCTION}

Given a real analytic function $f: U \rightarrow \mathbb{R}$ defined on an open neighborhood $U$ of $[-1,1]^{n}$, define $\tilde{f}: \mathbb{R}^{n} \rightarrow \mathbb{R}$ by

$$
\widetilde{f}(x)= \begin{cases}f(x), & \text { if } x \in[-1,1]^{n}, \\ 0, & \text { otherwise. }\end{cases}
$$

Any such function $\tilde{f}$ is called a restricted analytic function. Consider the structure $\mathbb{R}_{\text {an }}$, the expansion of the real ordered field by all restricted analytic functions, and denote its language by $\mathcal{L}_{\text {an }}$.

The definable sets of $\mathbb{R}_{a n}$ are known as the globally subanalytic sets and have been extensively studied by both geometers and model theorists alike. Gabrielov's theorem of the complement [9] shows that $\mathbb{R}_{\text {an }}$ is model complete, that is, all of its definable sets are projections of quantifier free definable sets. Denef and Van den Dries [8] strengthened Gabrielov's result by using Weierstrass preparation and Tarski's theorem to show that the expansion of $\mathbb{R}_{\text {an }}$ by division admits quantifier elimination. Van den Dries, Macintyre and Marker 23] then showed that if not only division but also all $n$th roots are added to the language (to obtain the structure we shall denote by $\mathbb{R}_{\text {an }}^{\mathbb{Q}}$ with language $\mathcal{L}_{\text {an }}^{\mathbb{Q}}$ ), then the theory is universally axiomatizable. Coupling this with the quantifier elimination shows by a simple model theoretic argument that all globally subanalytic functions are piecewise given by $\mathcal{L}_{\text {an }}^{\mathbb{Q}}$-terms. They then used this result to show that $\mathbb{R}_{\text {an }}(\exp , \log )$, the expansion of $\mathbb{R}_{\text {an }}$ by the unrestricted exponential and logarithm functions, admits quantifier elimination and is universally axiomatizable, and hence its definable functions are also piecewise given by terms. C. Miller [17] generalized the result for $\mathbb{R}_{\text {an }}^{\mathbb{Q}}$ by showing that $\mathbb{R}_{\text {an }}^{K}$ admits quantifier elimination and is universally axiomatizable, where $K$ is an arbitrary subfield of the reals and $\mathbb{R}_{\text {an }}^{K}$ is the expansion of $\mathbb{R}_{\text {an }}$ by all power functions $x \mapsto x^{r}: \mathbb{R}^{>0} \rightarrow \mathbb{R}^{>0}$ for $r \in K$.

The fact that the definable functions of $\mathbb{R}_{\text {an }}^{K}$ and $\mathbb{R}_{\mathrm{an}}(\exp , \log )$ are piecewise given by terms are fundamental geometric results, and thus one would expect that they should have purely geometric proofs with no recourse to model theoretic techniques.

Received by the editors August 20, 2004.

2000 Mathematics Subject Classification. Primary 03C10, 14P15; Secondary 03 C64.

Key words and phrases. Subanalytic preparation theorem, Weierstrass preparation.

(C)2006 American Mathematical Society 
Lion and Rolin [13] supplied such proofs by showing that these structures have preparation theorems. Here we shall only discuss the preparation theorem for $\mathbb{R}_{\mathrm{an}}^{K}$. (See [13] or Speissegger and Van den Dries 24] for the preparation theorem for $\mathbb{R}_{\mathrm{an}}(\mathrm{exp}, \log )$.) The preparation theorem for $\mathbb{R}_{\mathrm{an}}^{K}$ states that given any function $f(x, y)$ definable in $\mathbb{R}_{\text {an }}^{K}$, where $x$ ranges over $\mathbb{R}^{n}$ and $y$ over $\mathbb{R}, \mathbb{R}^{n+1}$ can be covered by finitely many quantifier free definable sets of a certain form such that on each of these sets,

$$
f(x, y)=a(x)|y-\theta(x)|^{r} u(x, y)
$$

for some $r \in K$ and $\mathcal{L}_{\text {an }}^{K}$-terms $a(x), \theta(x)$ and $u(x, y)$, where $u(x, y)$ is a unit of a certain form. When $f$ is written as such we say that $f$ is " $\mathcal{L}_{\text {an }}^{K}$-prepared".

The question behind this paper is whether the entire system of restricted analytic functions is really needed to obtain the preparation theorem. For any collection $\mathcal{F}$ of real analytic germs and subfield $K$ of the reals, one can ask whether every function definable in $\mathbb{R}_{\mathcal{F}}^{K}$ is $\mathcal{L}_{\mathcal{F}}^{K}$-prepared, where $\mathbb{R}_{\mathcal{F}}^{K}$ is the expansion of the real ordered field by all restricted " $\mathcal{F}$-analytic" functions and power functions $x^{r}$ for $r \in K$. It would be desirable for the members of $\mathcal{F}$ to be represented by some finite amount of information, such as being the solutions to some polynomial algebraic equations, or even more interestingly, some polynomial differential equations. With this motivation in mind we consider a Weierstrass system $\mathcal{F}$, of which the system of algebraic analytic germs and the system of differentially algebraic analytic germs are examples. A Weierstrass system is a collection of $\mathbb{R}$-algebras of real analytic germs containing the coordinate projections and closed under composition and Weierstrass preparation. (See Section 2 for a precise definition.)

Main Theorem. If $\mathcal{F}$ is a Weierstrass system, then every function definable in $\mathbb{R}_{\mathcal{F}}$ is $\mathcal{L}_{\mathcal{F}}^{\mathbb{Q}}$-prepared. More generally, if $K$ is a subfield of the field of exponents of $\mathcal{F}$, then every function definable in $\mathbb{R}_{\mathcal{F}}^{K}$ is $\mathcal{L}_{\mathcal{F}}^{K}$-prepared.

A set or function is called sub-Pfaffian if it is definable in the expansion of the real field by the collection of restricted Pfaffian functions [10. Gabrielov has asked whether the collection of sub-Pfaffian functions has a preparation theorem in the sense of Lion and Rolin. The Main Theorem provides a partial answer: sub-Pfaffian functions may be prepared within the larger system of restricted differentially algebraic functions. Whether or not the sub-Pfaffian functions have a preparation theorem themselves can be viewed as a particular case of the open question of whether or not $\mathbb{R}_{\mathcal{F}}$ has a preparation theorem for any quasianalytic class $\mathcal{F}$ closed under composition, division by variables and implicit functions. This is an axiomatic framework used by Rolin, Speissegger and Wilkie [21] to study the sets definable from quasianalytic Denjoy-Carleman classes 1 Because these classes are not closed under Weierstrass preparation [6], we shall be explicit in pointing out the step in our proof that uses Weierstrass preparation. The rest of the proof goes through under the weaker framework.

A detailed outline of the proof of the Main Theorem is postponed until the end of Section 3 so that we may first give all the definitions required for a precise

\footnotetext{
${ }^{1}$ During the refereeing and subsequent revising of this paper, I learned that Rambaud proves in his thesis 20] that if $\mathcal{F}$ is such a quasianalytic class, then $\mathbb{R}_{\mathcal{F}}^{\mathbb{Q}}$ has quantifier elimination and a universal axiomatization, and therefore its definable functions are piecewise given by terms. Combining this with 24] shows that $\mathbb{R}_{\mathcal{F}}$ has a preparation theorem in the sense given here, except that the units $u(x, y)$ appearing in the expressions $f(x, y)=a(x)|y-\theta(x)|^{r} u(x, y)$ are not required to be of any particular form.
} 
understanding of its statement. We begin by fixing some notation to be used throughout.

Notation. Given a set $A \subseteq \mathbb{R}^{n}, \operatorname{int}(A)$ and $\operatorname{cl}(A)$ denote the interior and closure of A.

The set of natural numbers $\{0,1,2, \ldots\}$ is denoted by $\mathbb{N}$. The letters $i, j, k, l, m, n$ and $d$ always range over $\mathbb{N}$, and $\alpha=\left(\alpha_{1}, \ldots, \alpha_{n}\right), \beta=\left(\beta_{1}, \ldots, \beta_{n}\right)$ and $\gamma=$ $\left(\gamma_{1}, \ldots, \gamma_{n}\right)$ range over $\mathbb{N}^{n}$, unless specified otherwise.

When $n$ is given, $x$ denotes the tuple of variables $\left(x_{1}, \ldots, x_{n}\right)$, and $x^{\alpha}:=$ $x_{1}^{\alpha_{1}} \cdots x_{n}^{\alpha_{n}}$. The letters $y$ and $y_{1}, y_{2}, y_{3}, \ldots$ denote single variables, if not specified otherwise. We denote the coordinate projection $\left(x, y_{1}, \ldots, y_{m}\right) \mapsto x$ by $\Pi_{n}$ : $\mathbb{R}^{n+m} \rightarrow \mathbb{R}^{n}$. We shall frequently work over $\mathbb{R}^{n+1}$ using the variables $(x, y)$.

For any differentiable map $f=\left(f_{1}, \ldots, f_{m}\right): \mathbb{R}^{n+m} \rightarrow \mathbb{R}^{m}$ and tuple of variables $z=\left(z_{1}, \ldots, z_{m}\right)$, we denote the matrix $\left(\frac{\partial f_{i}}{\partial z_{j}}(x, z)\right)_{i, j=1}^{m}$ by $\frac{\partial f}{\partial z}(x, z)$.

\section{TERMinOLGY FROM MODEL THEORY}

The point of Lion and Rolin's preparation theorem is to prove a model theoretic result without using any nonconstructive model theoretic techniques. So this paper does not need to assume that the reader has any background in model theory. But, since the very statement of the problem is model theoretic, it is most convenient to use some of its most basic terminology, which we now recall.

Consider a language $\mathcal{L}$ and an $\mathcal{L}$-structure $\mathcal{R}$ on $\mathbb{R}$. This means the following: $\mathcal{R}$ consists of the set of real numbers $\mathbb{R}$, called the "universe" of $\mathcal{R}$, along with a collection of distinguished subsets of $\mathbb{R}^{n}$, called " $n$-ary relations", and distinguished functions from $\mathbb{R}^{n}$ to $\mathbb{R}$, called " $n$-ary functions", for possibly various $n \in \mathbb{N}$. This collection of distinguished relations and functions is called the "signature" of $\mathcal{R}$. The functions in the signature of $\mathcal{R}$ are total, that is, defined on all of $\mathbb{R}^{n}$. Also, by convention $\mathbb{R}^{0}:=\{0\}$, and we identify the 0 -ary function $0 \mapsto r$ with the real number $r$ and call it a "constant". The "language" $\mathcal{L}$ of $\mathcal{R}$ is simply a collection of names for the members of the signature of $\mathcal{R}$, that is, a collection of symbols placed in a one-to-one correspondence with the signature of $\mathcal{R}$.

A string of symbols is called an " $\mathcal{L}$-term" if it is built from the function symbols of $\mathcal{L}$, parentheses ( and ), and an infinite list of variables $x_{1}, x_{2}, x_{3}, \ldots$, and if it can be constructed in the following inductive manner:

(i) any constant symbol in $\mathcal{L}$ is an $\mathcal{L}$-term;

(ii) any variable $x_{1}, x_{2}, x_{3}, \ldots$ is an $\mathcal{L}$-term;

(iii) if $f$ is an $m$-ary function symbol in $\mathcal{L}$ and $t_{1}, \ldots, t_{m}$ are $\mathcal{L}$-terms, then the string of symbols

$$
f\left(t_{1}, \ldots, t_{m}\right)
$$

is an $\mathcal{L}$-term.

In the obvious manner, every $\mathcal{L}$-term names a function obtained by composing the coordinate projection functions, $x \mapsto x_{i}$ for $i=1, \ldots, n$, with functions in the signature of $\mathbb{R}$.

In this paper the fact that $\mathcal{L}$ is a collection of names for the members of the signature of $\mathcal{R}$ is an unneeded formality: for any particular language $\mathcal{L}$ we shall only consider one $\mathcal{L}$-structure $\mathcal{R}$, so we shall abuse model theoretic terminology and simply consider $\mathcal{L}$ to be the signature of $\mathcal{R}$. Also, many different $\mathcal{L}$-terms can name the same function (for example, if $\mathcal{L}$ contains names for all rational power 
functions, then $\left(x_{1}^{2}\right)^{1 / 2}$ and $\left(x_{1}^{4}\right)^{1 / 4}$ are different $\mathcal{L}$-terms naming the same function $\left.x_{1} \mapsto\left|x_{1}\right|\right)$, but we shall not be concerned with this issue. So we shall further abuse model theoretic terminology and identify all $\mathcal{L}$-terms with the functions they name, thereby arriving at the following definition.

Definition 1.1. An $\mathcal{L}$-term is a function that can be obtained by composing coordinate projection functions with functions in the signature of $\mathcal{R}$. A function $f: \mathbb{R}^{n} \rightarrow \mathbb{R}$ is said to be piecewise given by $\mathcal{L}$-terms if there are finitely many $\mathcal{L}$-terms $t_{1}(x), \ldots, t_{k}(x)$ such that for each $a \in \mathbb{R}^{n}$ there exists $i \in\{1, \ldots, k\}$ such that $f(a)=t_{i}(a)$.

Note that because all functions in the signature of $\mathcal{R}$ are total, all $\mathcal{L}$-terms are total.

Example 1.2. For an example of this framework take $\mathcal{L}$ to be $\bar{L}_{\mathbb{R}}:=\{<,+, \cdot,-, r\}_{r \in \mathbb{R}}$ and $\mathcal{R}$ to be $\overline{\mathbb{R}}_{\mathbb{R}}$, the expansion of the real ordered field by all real constants. This structure consists of the set $\mathbb{R}$ as its universe, along with the distinguished relation $\left\{(x, y) \in \mathbb{R}^{2}: x<y\right\}$, distinguished functions $(x, y) \mapsto x+y,(x, y) \mapsto x y, x \mapsto-x$, and a constant $r$ for each $r \in \mathbb{R}$. The set of $\bar{L}_{\mathbb{R}}$-terms consists of all polynomial functions with real coefficients.

We shall only work with $\mathcal{L}$-structures $\mathcal{R}$ which are "expansions" of $\overline{\mathbb{R}}_{\mathbb{R}}$ by functions: this means the signature of $\mathcal{R}$ contains the signature of the real ordered field and all real constants, along with possibly more distinguished functions.

A subset of $\mathbb{R}^{n}$ is called " $\mathcal{L}$-atomic" if it is of the form $\left\{x \in \mathbb{R}^{n}: f(x)=0\right\}$ or $\left\{x \in \mathbb{R}^{n}: f(x)>0\right\}$ for some $\mathcal{L}$-term $f$. A subset of $\mathbb{R}^{n}$ which is a finite boolean combination of $\mathcal{L}$-atomic sets is called "quantifier free $\mathcal{L}$-definable". A set obtained from $\mathcal{L}$-atomic sets through the finite use of boolean operations and coordinate projections is called " $\mathcal{L}$-definable". A function $f: D \rightarrow \mathbb{R}^{m}$, where $D \subseteq \mathbb{R}^{n}$, is called (quantifier free) $\mathcal{L}$-definable if its graph is (quantifier free) $\mathcal{L}$-definable. The structure $\mathcal{R}$ has "quantifier elimination" if every $\mathcal{L}$-definable set is quantifier free $\mathcal{L}$-definable.

The quantifier free definable sets of $\overline{\mathbb{R}}_{\mathbb{R}}$ are commonly called the semialgebraic sets, and by Tarski's theorem $\overline{\mathbb{R}}_{\mathbb{R}}$ has quantifier elimination.

\section{WEIERSTRASS SYSTEMS}

For any point $a \in \mathbb{R}^{n}$ we can define an equivalence relation $\sim_{a}$ on the set of all functions defined in some neighborhood of $a$ by saying that $f \sim_{a} g$ if and only if $f(x)=g(x)$ on some sufficiently small neighborhood of $a$. The germ of $f$ at $a$ is defined to be the $\sim_{a}$-equivalence class of $f$. Any function which is the member of a germ is called a representative of the germ.

We will not distinguish between germs and functions in our notation, using single letters such as $f, g$ and $h$ to denote both germs and functions, and using $f+g, f g$ and $f \circ g$ to denote the operations of addition, multiplication and composition of germs and of functions. Since any statement about germs can be reinterpreted as a statement about functions by simply taking representatives and sufficiently small neighborhoods, using the same notation facilitates this transition from germs to functions.

A germ of functions at a point $a \in \mathbb{R}^{n}$ is called real analytic if it has a representative which is real analytic at $a$. We write $\mathcal{O}_{n}$ for the collection of all real analytic 
germs at the origin in $\mathbb{R}^{n}$, and also write $\mathcal{O}:=\bigcup_{n \in \mathbb{N}} \mathcal{O}_{n}$. An important property of $\mathcal{O}$ is the Weierstrass preparation theorem: for all $f(x, y) \in \mathcal{O}_{n+1}$ "regular in $y$ of order $d^{\prime \prime}$, meaning that $f(0)=\cdots=\frac{\partial^{d-1} f}{\partial y^{d-1}}(0)=0$ and $\frac{\partial^{d} f}{\partial y^{d}}(0) \neq 0$, there exist unique $w_{0}, \ldots, w_{d-1} \in \mathcal{O}_{n}$ and $u \in \mathcal{O}_{n+1}$ such that $w_{0}(0)=\cdots=w_{d-1}(0)=0$, $u(0) \neq 0$ and

$$
f(x, y)=\left(y^{d}+w_{d-1}(x) y^{d-1}+\cdots+w_{0}(x)\right) u(x, y) .
$$

When $f$ is written as such we say that $f$ is "Weierstrass prepared" and that $w(x, y):=y^{d}+w_{d-1}(x) y^{d-1}+\cdots+w_{0}(x)$ is the "Weierstrass polynomial" of $f$.

Definition 2.1. An analytic system of germs is a collection $\mathcal{F}=\bigcup_{n \in \mathbb{N}} \mathcal{F}_{n}$, where $\mathcal{F}_{0}=\mathbb{R}$ and for each $n>0, \mathcal{F}_{n}$ is an $\mathbb{R}$-algebra of real analytic germs at the origin in $\mathbb{R}^{n}$ which contains the germs at 0 of the coordinate projection functions $x \mapsto x_{i}$ for $i=1, \ldots, n$.

Given an analytic system of germs $\mathcal{F}$, a function $f: U \rightarrow \mathbb{R}^{m}$ defined on an open set $U \subseteq \mathbb{R}^{n}$ is called $\mathcal{F}$-analytic if $\left(\mathcal{F}_{n}\right)^{m}$ contains the germ of $f$ at each point of $U$. (More generally, for any set $A \subseteq \mathbb{R}^{n}$ we shall say that a function $f: A \rightarrow \mathbb{R}^{m}$ is $\mathcal{F}$ analytic if it extends to an $\mathcal{F}$-analytic function on an open neighborhood of $A$.) The structure $\mathbb{R}_{\mathcal{F}}$ is the expansion of the real ordered field by all functions $\tilde{f}: \mathbb{R}^{n} \rightarrow \mathbb{R}$ for which there exists an $\mathcal{F}$-analytic function $f: U \rightarrow \mathbb{R}$ on a neighborhood $U$ of $[-1,1]^{n}$ such that

$$
\widetilde{f}(x)= \begin{cases}f(x), & x \in[-1,1]^{n}, \\ 0, & \text { otherwise. }\end{cases}
$$

We call $\widetilde{f}$ a restricted $\mathcal{F}$-analytic function. For any $K \subseteq \mathbb{R}$ the structure $\mathbb{R}_{\mathcal{F}}^{K}$ is the expansion of $\mathbb{R}_{\mathcal{F}}$ by all power functions

$$
x \mapsto \begin{cases}x^{r}, & \text { for } x \geq 0, \\ 0, & \text { otherwise }\end{cases}
$$

for $r \in K$. The languages of $\mathbb{R}_{\mathcal{F}}$ and $\mathbb{R}_{\mathcal{F}}^{K}$ are denoted by $\mathcal{L}_{\mathcal{F}}$ and $\mathcal{L}_{\mathcal{F}}^{K}$, respectively.

An analytic system of germs $\mathcal{F}$ is called a Weierstrass system if it is closed under composition and Weierstrass preparation, as defined below:

(i) Composition $=($ Composition of Germs $)+($ Local Translation $)$ :

(a) Composition of Germs: for all $f \in \mathcal{F}_{m}$ and $g \in\left(\mathcal{F}_{n}\right)^{m}$ such that $g(0)=$ $0, f \circ g \in \mathcal{F}_{n}$.

(b) Local Translation: every member of $\mathcal{F}_{n}$ contains a representative $f$ : $U \rightarrow \mathbb{R}$ such that $\mathcal{F}_{n}$ contains the germ of $f$ at each point of $U$.

(ii) Weierstrass Preparation: if $f(x, y) \in \mathcal{F}_{n+1}$ is regular in $y$ of order $d$, and if

$$
f(x, y)=\left(y^{d}+w_{d-1}(x) y^{d-1}+\cdots+w_{0}(x)\right) u(x, y)
$$

is the Weierstrass prepared form of $f$, then $w_{0}, \ldots, w_{d-1} \in \mathcal{F}_{n}$ and $u \in \mathcal{F}_{n+1}$.

(See Section 9 for a comparison of Weierstrass systems with Van den Dries's nearly identical notion of a "convergent Weierstrass system" [22.)

Examples 2.2. Clearly $\mathcal{O}$ is the largest Weierstrass system. We say that $f \in \mathcal{O}_{n}$ is algebraic (over $\mathbb{R}[x]$ ) if $p(x, f(x))=0$ for some nonzero real polynomial $p(x, y)$. We say that $f \in \mathcal{O}_{n}$ is differentially algebraic (over $\mathbb{R}$ ) if the field extension $\mathbb{R} \subseteq \mathbb{R}\left(\frac{\partial^{|\alpha|} f}{\partial x^{\alpha}}\right)_{\alpha \in \mathbb{N}^{n}}$ has finite transcendence degree. The smallest Weierstrass system is

$$
\mathcal{A}:=\{f \in \mathcal{O}: f \text { is algebraic }\},
$$


and a Weierstrass system strictly containing $\mathcal{A}$ and strictly contained in $\mathcal{O}$ is

$$
\mathcal{D}:=\{f \in \mathcal{O}: f \text { is differentially algebraic }\} .
$$

Proof. See Bochnak, Coste and Roy [5, Section 8.2] and Van den Dries [22, Section $5]$ for proofs that $\mathcal{A}$ and $\mathcal{D}$ are Weierstrass systems, respectively.

Van den Dries 22, Section 1] remarks that $\mathcal{A}$ is the smallest Weierstrass system (though using a slightly different definition). This fact can be proved as follows. Let $\mathcal{S}$ be the intersection of all Weierstrass systems. Clearly $\mathcal{S}$ is the smallest Weierstrass system. To show that $\mathcal{A}=\mathcal{S}$ it suffices to show that $\mathcal{A} \subseteq \mathcal{S}$, so fix $f \in \mathcal{A}_{n}$. It follows from [5, Theorem 8.1.4] that $f(x)=p(x, g(x))$, where

(i) $p(x, z)$ is the germ of a real polynomial in $(x, z)$, where $z=\left(z_{1}, \ldots, z_{m}\right)$ for some $m$;

(ii) $g(x)=\left(g_{1}(x), \ldots, g_{m}(x)\right)$ is the analytic germ implicitly defined by the conditions $g(0)=0$ and $q(x, g(x))=0$ for some $q(x, z) \in(\mathbb{R}[x, z])^{m}$ such that $q(0)=0$ and the matrix $\frac{\partial q}{\partial z}(0)$ is nonsingular.

But $\mathcal{S}$ contains all polynomials and is closed under a composition of germs and under implicit functions (see Remarks 2.3), so $f \in \mathcal{S}_{n}$. Hence $\mathcal{A}=\mathcal{S}$.

The fact that the exponential function is differentially algebraic and transcendental shows that the inclusion $\mathcal{A} \subseteq \mathcal{D}$ is proper. To show that the inclusion $\mathcal{D} \subseteq \mathcal{O}$ is proper we point out two sources of algebraic analytic functions which are not differentially algebraic: Mahler [16, Section 137 (Theorem of J. Popken)] shows that a one-variable power series $f(x)=\sum_{k=0}^{\infty} f_{k} x^{k}$ will not be differentially algebraic if its coefficients are algebraic and tend to 0 too quickly, and Lipshitz and Rubel [15] show that $f$ will not be differentially algebraic if the size of the "gaps" in its support $\left\{k \in \mathbb{N}: f_{k} \neq 0\right\}$ grow too quickly.

We now gather together in Remarks 2.3 some basic properties of Weierstrass systems we shall need. In each remark we are explicit with our hypotheses in order to emphasize the relationships between the various closure properties of Weierstrass systems.

Remarks 2.3. Consider an analytic system of germs $\mathcal{F}$.

1. If $\mathcal{F}$ is closed under Weierstrass preparation, then $\mathcal{F}$ is "closed under implicit functions":

If $f \in \mathcal{F}_{n+1}$ is such that $f(0)=0$ and $\frac{\partial f}{\partial y}(0) \neq 0$, and if $g \in \mathcal{O}_{n}$ is implicitly defined by the conditions $g(0)=0$ and $f(x, g(x))=0$, then $g \in \mathcal{F}_{n}$.

Proof. Obvious.

If $\mathcal{F}$ is closed under Weierstrass preparation and a composition of germs, then $\mathcal{F}$ is "closed under division by variables":

If $f \in \mathcal{F}_{n+1}$ is such that $f(x, 0)=0$, then $f(x, y)=y g(x, y)$ from some $g \in \mathcal{F}_{n+1}$.

Proof. Fix $\lambda \in \mathbb{R}^{n}$ such that $f(x+\lambda y, y)$ is regular in $y$, apply Weierstrass preparation to $f(x+\lambda y, y)$, divide by $y$, and then apply the inverse transformation $(x, y) \mapsto(x-\lambda y, y)$. 
2. If $\mathcal{F}$ is closed under a composition of germs and under implicit functions (as defined in the preceding remark), then $\mathcal{F}$ satisfies the following more general version of the implicit function theorem:

If $f=\left(f_{1}, \ldots, f_{m}\right) \in\left(\mathcal{F}_{n+m}\right)^{m}$ is such that $f(0)=0$ and $\operatorname{det} \frac{\partial f}{\partial z}(0) \neq 0$, where $z=\left(z_{1}, \ldots, z_{m}\right)$, and if $g \in\left(\mathcal{O}_{n}\right)^{m}$ is implicitly defined by the conditions $g(0)=0$ and $f(x, g(x))=0$, then $g \in\left(\mathcal{F}_{n}\right)^{m}$.

Proof. We proceed by induction on $m$. The case $m=1$ is assumed, so consider $m>1$. Since $\frac{\partial f}{\partial z}(0)$ is nonsingular, by possibly permuting the components of $f$ we may assume that $\frac{\partial f_{m}}{\partial z_{m}}(0) \neq 0$ and that $\frac{\partial f^{\prime}}{\partial z^{\prime}}(0)$ is nonsingular, where $f^{\prime}=\left(f_{1}, \ldots, f_{m-1}\right)$ and $z^{\prime}=\left(z_{1}, \ldots, z_{m-1}\right)$. Define $\lambda=$ $\left(\lambda_{1}, \ldots, \lambda_{m-1}\right)$ by $\lambda_{i}=-\frac{\partial f_{m}}{\partial z_{i}}(0) / \frac{\partial f_{m}}{\partial z_{m}}(0)$ for $i=1, \ldots, m-1$, and let $L(x, z):=\left(x, z^{\prime}, z_{m}+\lambda \cdot z^{\prime}\right)$, where $\lambda \cdot z^{\prime}$ denotes the scalar product. Then

$$
\frac{\partial\left(f_{m} \circ L\right)}{\partial z_{m}}(0) \neq 0 \text { and } \frac{\partial\left(f_{m} \circ L\right)}{\partial z_{i}}(0)=0 \text { for } i=1, \ldots, m-1 .
$$

Because the matrix $\frac{\partial f}{\partial z}(0)$ and linear map $L$ are both nonsingular, $\frac{\partial(f \circ L)}{\partial z}(0)$ is nonsingular. Moreover, the determinant of this matrix is the product of $\frac{\partial\left(f_{m} \circ L\right)}{\partial z_{m}}(0)$ and $\operatorname{det} \frac{\partial\left(f^{\prime} \circ L\right)}{\partial z^{\prime}}(0)$, so $\frac{\partial\left(f^{\prime} \circ L\right)}{\partial z^{\prime}}(0)$ is nonsingular. Therefore by the induction hypothesis we may fix $h \in\left(\mathcal{F}_{n+1}\right)^{m-1}$ such that $h(0)=0$ and $f^{\prime} \circ L\left(x, h\left(x, z_{m}\right), z_{m}\right)=0$. Put $F\left(x, z_{m}\right):=f_{m} \circ L\left(x, h\left(x, z_{m}\right), z_{m}\right)$ and observe that by (2.1), $\frac{\partial F}{\partial z_{m}}(0)=\frac{\partial\left(f_{m} \circ L\right)}{\partial z_{m}}(0) \neq 0$, so we may fix $H \in \mathcal{F}_{n}$ such that $H(0)=0$ and $F(x, H(x))=0$. Therefore the germ $g(x)$ satisfying $g(0)=0$ and $f(x, g(x))=0$ is given by

$$
g(x)=(h(x, H(x)), H(x)+\lambda \cdot h(x, H(x))),
$$

which is in $\left(\mathcal{F}_{n}\right)^{m}$.

3. If $\mathcal{F}$ is closed under division by variables and a composition of germs, then $\mathcal{F}$ is closed under differentiation.

Proof. Let $f(x, y) \in \mathcal{F}_{n+1}$; we show that $\frac{\partial f}{\partial y} \in \mathcal{F}_{n+1}$. Define $D(x, y, z):=$ $f(x, y+z)-f(x, y)$. Then $D(x, y, 0)=0$, so $D(x, y, z)=z g(x, y, z)$ for some $g \in \mathcal{F}_{n+2}$. Hence $\frac{\partial f}{\partial y}(x, y)=g(x, y, 0)$ is in $\mathcal{F}_{n+1}$.

4. If $\mathcal{F}$ is closed under implicit functions, then $\mathcal{F}$ is closed under "multiplicative inverses" and "radicals of positive units":

(a) If $f \in \mathcal{F}_{n}$ is such that $f(0) \neq 0$, then $1 / f \in \mathcal{F}_{n}$.

(b) If $k$ is a positive integer and $f \in \mathcal{F}_{n}$ is such that $f(0)>0$, then $f^{1 / k} \in \mathcal{F}_{n}$.

If $\mathcal{F}$ is also closed under a composition of germs, then more generally,

$\left(\mathrm{b}^{\prime}\right)$ if $0 \neq r \in \mathbb{R}$ and $f \in \mathcal{F}_{n}$ are such that $\left(1+x_{1}\right)^{r} \in \mathcal{F}_{1}$ and $f(0)>0$, then $f^{1 / r} \in \mathcal{F}_{n}$. 
Proof. The germs $1 / f(x)-1 / f(0), f(x)^{1 / k}-f(0)^{1 / k}$ and $f(x)^{1 / r}-f(0)^{1 / r}$ are implicitly defined by the following nonsingular equations:

$$
\begin{aligned}
(y+1 / f(0)) f(x)-1 & =0, \\
\left(y+f(0)^{1 / k}\right)^{k}-f(x) & =0 \\
f(0)\left(\frac{y}{f(0)^{1 / r}}+1\right)^{r}-f(x) & =0 .
\end{aligned}
$$

5. If $\mathcal{F}$ is closed under composition of germs and implicit functions, it follows easily from the preceding remark that $\left\{r \in \mathbb{R}:\left(1+x_{1}\right)^{r} \in \mathcal{F}_{1}\right\}$ is a field, which we shall call the field of exponents of $\mathcal{F}$.

\section{LION-ROLIN PREPARATION}

From now through Section 7 fix a Weierstrass system $\mathcal{F}$ and a field $K \subseteq \mathbb{R}$.

Definition 3.1. A set $C \subseteq \mathbb{R}^{n+1}$ is an $\mathcal{L}_{\mathcal{F}}^{K}$-cylinder if $B:=\Pi_{n}(C)$ is a quantifier free $\mathcal{L}_{\mathcal{F}}^{K}$-definable set, called the base of $C$, and $C=\{(x, y) \in B \times \mathbb{R}: \varphi(x, y)\}$, where $\varphi(x, y)$ is a formula either of the form

$$
y=t(x),
$$

or of one of the forms

$$
y<s(x), \quad s(x)<y<t(x), \text { or } t(x)<y,
$$

for $\mathcal{L}_{\mathcal{F}}^{K}$-terms $s(x)$ and $t(x)$ which are $\mathcal{F}$-analytic on $B$. We say that $C$ is thin if $\varphi(x, y)$ is as in (3.1) and that $C$ is fat if $\varphi(x, y)$ is as in (3.2). Furthermore, by induction on $n$ we say that the cylinder $C \subseteq \mathbb{R}^{n+1}$ is an $\mathcal{L}_{\mathcal{F}}^{K}$-term cell if

(i) when $n=0, C$ is a point or an open interval;

(ii) when $n>0$, its base $B$ is an $\mathcal{L}_{\mathcal{F}}^{K}$-term cell.

A finite collection $\mathcal{C}$ of $\mathcal{L}_{\mathcal{F}}^{K}$-term cells in $\mathbb{R}^{n}$ is a decomposition of $\mathbb{R}^{n}$ if $\left\{\Pi_{k}(C)\right.$ : $C \in \mathcal{C}\}$ is a partition of $\mathbb{R}^{k}$ for $k=1, \ldots, n$. A partition $\mathcal{C}$ of $\mathbb{R}^{n}$ is compatible with a collection $\mathcal{D}$ of subsets of $\mathbb{R}^{n}$ if for all $C \in \mathcal{C}$ and all $D \in \mathcal{D}$, either $C \subseteq D$ or $C \cap D=\emptyset$.

We shall frequently use the fact that the collection of subsets of $\mathbb{R}^{n+1}$ which are finite unions of $\mathcal{L}_{\mathcal{F}}^{K}$-cylinders is a boolean algebra.

Definition 3.2. A function $u: A \rightarrow \mathbb{R}$, where $A \subseteq \mathbb{R}^{n}$, is called a unit if $u$ has constant positive or negative sign on $A$. If the domain of $u$ needs specification, we say that $u$ is a "unit on $A . "$

Definition 3.3. Given a set $A \subseteq\left\{(x, y) \in \mathbb{R}^{n+1}: y \neq 0\right\}$ which is a finite union of $\mathcal{L}_{\mathcal{F}}^{K}$-cylinders, a function $F: A \rightarrow \mathbb{R}$ is called an $\mathcal{L}_{\mathcal{F}}^{K}$-special function if it can be written as $F=f \circ \varphi$, where

(i) $\varphi: A \rightarrow \mathbb{R}^{N+2 m}$ is a bounded $\mathcal{F}$-analytic function of the form

$$
\left(a_{1}(x), \ldots, a_{N}(x), b_{1}(x)|y|^{r_{1}}, \ldots, b_{m}(x)|y|^{r_{m}}, c_{1}(x)|y|^{-r_{1}}, \ldots, c_{m}(x)|y|^{-r_{m}}\right),
$$

where the $a_{i}(x), b_{i}(x), c_{i}(x)$ 's are all $\mathcal{L}_{\mathcal{F}}^{K}$-terms and the $r_{i}$ 's are all positive numbers in $K$;

(ii) $f: \mathbb{R}^{N+2 m} \rightarrow \mathbb{R}$ is $\mathcal{F}$-analytic on $\operatorname{cl}(\varphi(A))$. 
If $f$ is a unit on $\operatorname{cl}(\varphi(A))$, we call $F$ an $\mathcal{L}_{\mathcal{F}}^{K}$-special unit. As in the previous definition, we shall call $F$ an $\mathcal{L}_{\mathcal{F}}^{K}$-special function/unit "on $A$ " if the domain of $F$ needs specification.

If $K=\mathbb{Q}$ in Definition 3.3 , then by partitioning $A$ into smaller cylinders we can assume that $m=1$ and $r_{1}=1 / p$ for some positive integer $p$. More precisely, we have the following.

Lemma 3.4. Let $F: A \rightarrow \mathbb{R}$ be an $\mathcal{L}_{\mathcal{F}}^{\mathbb{Q}}$-special function. Then there is a finite cover $\mathcal{C}$ of $A$ by $\mathcal{L}_{\mathcal{F}}^{\mathbb{Q}}$-cylinders such that for each $C \in \mathcal{C}$ there is a bounded $\mathcal{F}$ analytic function $\psi: C \rightarrow \mathbb{R}^{M+2}$ of the form

$$
\psi(x, y)=\left(d_{1}(x), \ldots, d_{M}(x), d_{M+1}(x)|y|^{1 / p}, d_{M+2}(x)|y|^{-1 / p}\right)
$$

for some $\mathcal{L}_{\mathcal{F}}^{\mathbb{Q}}$-terms $d_{1}(x), \ldots, d_{M+2}(x)$ and positive integer $p$, and there is a function $g: \mathbb{R}^{M+2} \rightarrow \mathbb{R}$ which is $\mathcal{F}$-analytic on $\operatorname{cl}(\psi(C))$ and is such that $F=g \circ \psi$ on $C$.

Proof. We write $w$ for the tuple of variables $\left(w_{1}, \ldots, w_{N}\right)$ and use the notation of Definition 3.3. We show how to successively mutate $f, \varphi, N$ and $A$ into $g, \psi, M$ and $C$.

First, by considering each set

$$
\left\{(x, y) \in A: \operatorname{sign}\left(b_{i}(x)\right)=\sigma_{i}, \operatorname{sign}\left(c_{i}(x)\right)=\sigma_{i}^{\prime} \text { for } i=1, \ldots, m\right\}
$$

separately for all possible sign conditions $\sigma, \sigma^{\prime} \in\{-1,0,1\}^{m}$, we may assume that all the $b_{i}$ 's and $c_{i}$ 's have constant sign on $A$. Then by absorbing the signs of the $b_{i}$ 's and $c_{i}$ 's into $f$, we may assume that $b_{i}, c_{i}>0$ for all $i$.

Second, fix positive integers $q_{1}, \ldots, q_{m}, p$ such that $r_{i}=q_{i} / p$ for all $i$, and write $b_{i}(x)|y|^{q_{i} / p}=\left(b_{i}(x)^{1 / q_{i}}|y|^{1 / p}\right)^{q_{i}}$ and $c_{i}(x)|y|^{-q_{i} / p}=\left(c_{i}(x)^{1 / q_{i}}|y|^{-1 / p}\right)^{q_{i}}$. Therefore by replacing $f\left(w, y_{1}, \ldots, y_{m}, z_{1}, \ldots, z_{m}\right)$ with $f\left(w, y_{1}^{q_{1}}, \ldots, y_{m}^{q_{m}}, z_{1}^{q_{1}}, \ldots, z_{m}^{q_{m}}\right)$ and redefining $\varphi$ accordingly, we may assume that $r_{i}=1 / p$ for all $i$.

Finally, by considering each condition " $b_{i}(x) \geq b_{j}(x)$ for all $j$ " and " $c_{i}(x) \geq c_{j}(x)$ for all $j$ " separately for $i=1, \ldots, m$, and then reindexing in $i$, we may assume that $b_{1}(x) \geq b_{2}(x), \ldots, b_{m}(x)$ and $c_{1}(x) \geq c_{2}(x), \ldots, c_{m}(x)$ on $A$. For $i=2, \ldots, m$ write

$$
b_{i}(x)|y|^{1 / p}=\frac{b_{i}(x)}{b_{1}(x)} b_{1}(x)|y|^{1 / p}
$$

and

$$
c_{i}(x)|y|^{-1 / p}=\frac{c_{i}(x)}{c_{1}(x)} c_{1}(x)|y|^{-1 / p} .
$$

By extending the list $a_{1}, \ldots, a_{N}$ of bounded functions to include all the $b_{i} / b_{1}$ 's and $c_{i} / c_{1}$ 's, we may simply assume that $b_{1}=\cdots=b_{m}$ and $c_{1}=\cdots=c_{m}$. Modifying $f$ accordingly finishes the proof.

Definition 3.5. A function $f: \mathbb{R}^{n+1} \rightarrow \mathbb{R}$ is $\mathcal{L}_{\mathcal{F}}^{K}$-prepared on $A \subseteq \mathbb{R}^{n+1}$ if there is a finite cover $\mathcal{C}$ of $A$ by $\mathcal{L}_{\mathcal{F}}^{K}$-cylinders such that for each $C \in \mathcal{C}, f$ is of an " $\mathcal{L}_{\mathcal{F}}^{K}$-prepared form on $C$ ", meaning that either

(i) $C$ is thin, say $C=\{(x, y) \in B \times \mathbb{R}: y=t(x)\}$ for some $\mathcal{L}_{\mathcal{F}}^{K}$-term $t(x)$, and there is an $\mathcal{L}_{\mathcal{F}}^{K}$-term $s(x)$ such that $f(x, t(x))=s(x)$ for all $x \in B$, where both $t(x)$ and $s(x)$ are $\mathcal{F}$-analytic on $B$; or 
(ii) $C$ is fat and

$$
f(x, y)=a(x)|y-\theta(x)|^{r} u(x, y-\theta(x))
$$

on $C$, where $r \in K$, both $a(x)$ and $\theta(x)$ are $\mathcal{L}_{\mathcal{F}}^{K}$-terms which are $\mathcal{F}$-analytic on the base of $C, y \neq \theta(x)$ on $C$, and $u(x, y)$ is an $\mathcal{L}_{\mathcal{F}}^{K}$-special unit on $\{(x, y-\theta(x)):(x, y) \in C\}$.

If $A=\mathbb{R}^{n+1}$, we simply say that $f$ is $\mathcal{L}_{\mathcal{F}}^{K}$-prepared.

For $f$ and $C$ in the previous definition and $(x, y) \in C$, in case (i) the sign of $f(x, y)$ equals the sign of $s(x)$, and in case (ii) the sign of $f(x, y)$ equals the sign of $a(x)$, assuming, as we may, that $u$ is a positively valued unit. So by partitioning the base sets of the $C^{\text {'s, }}$, we can always assume that $f$ has constant sign on each $C \in \mathcal{C}$.

In the course of proving the preparation theorem it is convenient to distinguish what are essentially two special types of $\mathcal{L}_{\mathcal{F}}^{\mathbb{Q}}$-preparation. In the definition of " $f$ is $\mathcal{L}_{\mathcal{F}}^{\mathbb{Q}}$-prepared on $A^{\prime \prime}$, if instead of $(\underline{3.3)}$ we have

$$
f(x, y)=a(x)(y-\theta(x))^{d} u(x, y-\theta(x))
$$

on $C$, where $d \in \mathbb{Z}, a(x)$ and $\theta(x)$ are $\mathcal{L}_{\mathcal{F}}^{\mathbb{Q}}$-terms, and $u(x, y)$ is a "ZZZ-special unit", that is, of the form

$$
v\left(b_{1}(x), \ldots, b_{M}(x), b_{M+1}(x) y, b_{M+2}(x) y^{-1}\right)
$$

for $\mathcal{L}_{\mathcal{F}}^{\mathbb{Q}}$-terms $b_{1}, \ldots, b_{M+2}$ and $\mathcal{F}$-analytic unit $v$, then we say that $f$ is $\mathbb{Z}$-prepared on $A$. If we additionally require that $d \in \mathbb{N}$, we say that $f$ is $\mathbb{N}$-prepared on $A$. If $d \in \mathbb{N}$ and $b_{M+2}$ happens to be 0 , so we do not divide by $y-\theta(x)$, then we do not require that $y \neq \theta(x)$ on $C$. The concept of $\mathbb{N}$-preparation is a special case of $\mathbb{Z}$-preparation, and any function which is $\mathbb{Z}$-prepared can also be $\mathcal{L}_{\mathcal{F}}^{\mathbb{Q}}$-prepared by further partitioning the cylinders given by the $\mathbb{Z}$-preparation.

Example 3.6. The function $f: \mathbb{R}^{2} \rightarrow \mathbb{R}$ given by $f(x, y)=y^{2}-x$ is $\mathbb{N}$-prepared. Indeed, let $1<\delta<\epsilon$ and put

$$
\begin{aligned}
& C_{1}=\left\{(x, y) \in \mathbb{R}^{2}: x=0\right\}, \\
& C_{2}=\left\{(x, y) \in \mathbb{R}^{2}: x>0, y>\delta \sqrt{x}\right\}, \\
& C_{3}=\left\{(x, y) \in \mathbb{R}^{2}: x>0, \epsilon^{-1} \sqrt{x}<y<\epsilon \sqrt{x}\right\}, \\
& C_{4}=\left\{(x, y) \in \mathbb{R}^{2}: x>0,-\delta^{-1} \sqrt{x}<y<\delta^{-1} \sqrt{x}\right\}, \\
& C_{5}=\left\{(x, y) \in \mathbb{R}^{2}: x>0,-\epsilon \sqrt{x}<y<-\epsilon^{-1} \sqrt{x}\right\}, \\
& C_{6}=\left\{(x, y) \in \mathbb{R}^{2}: x>0, y<-\delta \sqrt{x}\right\}, \\
& C_{7}=\left\{(x, y) \in \mathbb{R}^{2}: x<0, y>\frac{1}{2} \sqrt{-x}\right\} \\
& C_{8}=\left\{(x, y) \in \mathbb{R}^{2}: x<0,-2 \sqrt{-x}<y<2 \sqrt{-x}\right\}, \\
& C_{9}=\left\{(x, y) \in \mathbb{R}^{2}: x<0, y<-\frac{1}{2} \sqrt{-x}\right\} .
\end{aligned}
$$




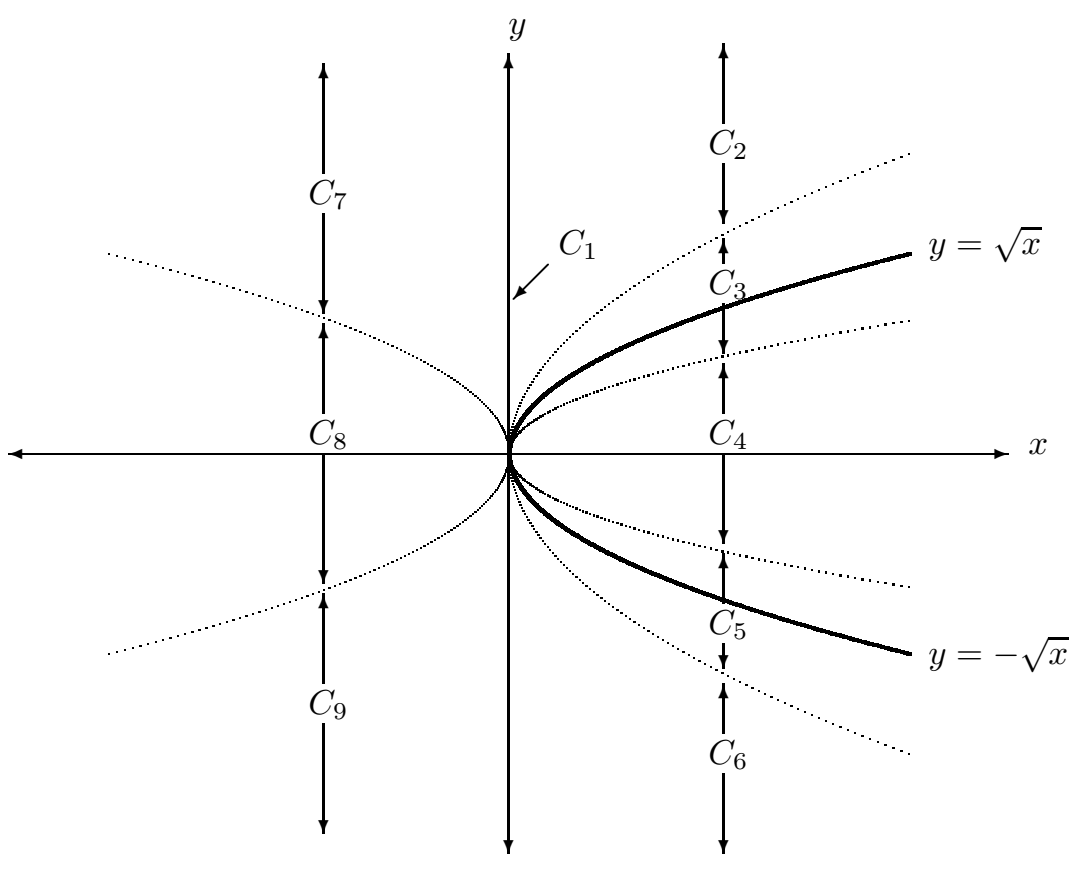

Figure 1. Zero set of $f(x, y)=y^{2}-x$, and cylinders $C_{1}, \ldots, C_{9}$.

Then $\mathcal{C}=\left\{C_{1}, \ldots, C_{9}\right\}$ is an $\mathcal{L}_{\mathcal{F}}^{\mathbb{Q}}$-cylinder covering of $\mathbb{R}^{2}$, and $f$ is of an $\mathbb{N}$-prepared form on each $C \in \mathcal{C}$ (in the following, the expressions in square brackets are positively valued $\mathbb{Z}$-special units):

$$
f(x, y)= \begin{cases}y^{2} & \text { on } C_{1}, \\ y^{2}\left[1-\frac{x}{y^{2}}\right] & \text { on } C_{2} \cup C_{6} \cup C_{7} \cup C_{9}, \\ -x\left[1-\frac{y^{2}}{x}\right] & \text { on } C_{4} \cup C_{8}, \\ \left.\sqrt{x}(y-\sqrt{x})\left[\frac{y-\sqrt{x}}{\sqrt{x}}+2\right]\right] & \text { on } C_{3}, \\ -\sqrt{x}(y+\sqrt{x})\left[-\frac{y+\sqrt{x}}{\sqrt{x}}+2\right] & \text { on } C_{5},\end{cases}
$$

See Figure 1 for a rough picture showing the zero set of $f$ and the cylinders $C_{1}, \ldots, C_{9}$. So as not to clutter the picture, it ignores the fact that the cylinders actually overlap.

The cylinders involving the zeros of $f$, namely $C_{3}$ and $C_{5}$, can be made as thin as we want by taking $\epsilon$ to be sufficiently close to 1 . A preparation with this property is called "tight", and the next section shows that any preparation can be modified into a tight preparation. 
This $\mathbb{N}$-preparation of $f$ can be made into an $\mathcal{L}_{\mathcal{F}}^{\mathbb{Q}}$-preparation by simply partitioning the cylinders $C_{3}$ and $C_{5}$. For instance, partition $C_{3}$ into

$$
\begin{aligned}
& C_{3,>}=\left\{(x, y) \in \mathbb{R}^{2}: x>0, \sqrt{x}<y<\epsilon \sqrt{x}\right\}, \\
& C_{3,=}=\left\{(x, y) \in \mathbb{R}^{2}: y=\sqrt{x}\right\}, \\
& C_{3,<}=\left\{(x, y) \in \mathbb{R}^{2}: x>0, \epsilon^{-1} \sqrt{x}<y<\sqrt{x}\right\},
\end{aligned}
$$

and note that

$$
f(x, y)=\left\{\begin{array}{lll}
\sqrt{x}|y-\sqrt{x}|\left[\frac{|y-\sqrt{x}|}{\sqrt{x}}+2\right] & \text { on } & C_{3,>}, \\
0 & \text { on } & C_{3,=}, \\
-\sqrt{x}|y-\sqrt{x}|\left[-\frac{|y-\sqrt{x}|}{\sqrt{x}}+2\right] & \text { on } & C_{3,<,},
\end{array}\right.
$$

where expressions in square brackets are $\mathcal{L}_{\mathcal{F}}^{\mathbb{Q}}$-units.

The Main Theorem states that if $\mathcal{F}$ is a Weierstrass system and $K$ is a subfield of the field of exponents of $\mathcal{F}$, then every function definable in $\mathbb{R}_{\mathcal{F}}^{K}$ is $\mathcal{L}_{\mathcal{F}}^{K}$-prepared. The first step in proving this is to see that to prepare all definable functions, it suffices to prepare all terms. In fact, if all terms are prepared, we easily obtain a very strong cell decomposition theorem.

Lemma 3.7. The following hold if every $\mathcal{L}_{\mathcal{F}}^{K}$-term is $\mathcal{L}_{\mathcal{F}}^{K}$-prepared:

(i) $\mathbb{R}_{\mathcal{F}}^{K}$ has quantifier elimination.

(ii) For any finite collection $\mathcal{D}$ of $\mathcal{L}_{\mathcal{F}}^{K}$-definable subsets of $\mathbb{R}^{n}$, there is an $\mathcal{L}_{\mathcal{F}}^{K}$ term cell decomposition of $\mathbb{R}^{n}$ compatible with $\mathcal{D}$.

(iii) All $\mathcal{L}_{\mathcal{F}}^{K}$-definable functions are piecewise given by $\mathcal{L}_{\mathcal{F}}^{K}$-terms and are $\mathcal{L}_{\mathcal{F}}^{K}$ prepared.

In the following proof, and in subsequent proofs and informal discussions in which the structure $\mathbb{R}_{\mathcal{F}}^{K}$ is clear from context, we shall frequently drop the prefix " $\mathcal{L}_{\mathcal{F}}^{K}$-" in our terminology and simply say "term", "cylinder", "special unit", "prepared", etc.

Proof. To show (i), consider terms $f_{1}, \ldots, f_{k}: \mathbb{R}^{n+1} \rightarrow \mathbb{R}$ and a sign condition $\sigma \in\{-1,0,1\}^{k}$, and let

$$
A:=\left\{(x, y) \in \mathbb{R}^{n+1}: \operatorname{sign} f_{i}(x, y)=\sigma_{i} \text { for } i=1, \ldots, k\right\} .
$$

It suffices to show that $\Pi_{n}(A)$ is quantifier free definable. By preparing each $f_{i}$, there are quantifier free definable sets $B_{1}, \ldots, B_{l}$ partitioning $\mathbb{R}^{n}$ and a finite collection $\mathcal{C}=\bigcup_{j=1}^{l} \mathcal{C}_{i}$ of cylinders partitioning $\mathbb{R}^{n+1}$ such that for each $i \in\{1, \ldots, k\}$, $j \in\{1, \ldots, l\}$ and $C \in \mathcal{C}_{j}, \Pi_{n}(C)=B_{j}$ and $f_{i}$ has constant sign on $C$. Therefore $A=\bigcup \mathcal{C}^{\prime}$ for some $\mathcal{C}^{\prime} \subseteq \mathcal{C}$, so $\Pi_{n}(A)=\bigcup_{i \in I} B_{i}$ for some $I \subseteq\{1, \ldots, l\}$.

To show (ii), by quantifier elimination it suffices to consider a finite collection $\mathcal{D}$ of quantifier free definable subsets of $\mathbb{R}^{n+1}$ and show by induction on $n$ that there is a term cell decomposition of $\mathbb{R}^{n+1}$ compatible with $\mathcal{D}$. Fix a finite partition $\mathcal{D}^{\prime}$ of $\mathbb{R}^{n+1}$ compatible with $\mathcal{D}$ such that each member of $\mathcal{D}^{\prime}$ is defined by a sign condition, such as the set $A$ above. By the above analysis, each member of $\mathcal{D}^{\prime}$ is a finite disjoint union of cylinders. We may inductively assume that there is a term cell decomposition of $\mathbb{R}^{n}$ compatible with $\left\{\Pi_{n}(D): D \in \mathcal{D}^{\prime}\right\}$, and hence via the cylinder partition of each $D \in \mathcal{D}^{\prime}$, this term cell decomposition lifts to a term cell decomposition of $\mathbb{R}^{n+1}$ compatible with $\mathcal{D}^{\prime}$, and so also with $\mathcal{D}$. 
To show (iii), let $f: \mathbb{R}^{n} \rightarrow \mathbb{R}$ be definable. From (ii) the graph of $f$ is a finite union of cylinders. Since $f$ is a function, each of these cylinders must be thin, so $f$ is piecewise given by terms. Since each of these terms are prepared, so is $f$.

Outline of the proof of the Main Theorem. The primary task at hand is to establish the Main Theorem in the special case of $K=\mathbb{Q}$. The theorem for a general $K$ follows from the theorem for $K=\mathbb{Q}$ and from some of the concepts developed in its proof.

Sections 4 and 5 present the arguments from [13] which reduce the problem of preparing all terms to that of preparing functions of the form $F(x, y)=f(x, g(x) / y, y)$ for $\mathcal{F}$-analytic functions $f$ and $g$. Section 4 shows that a finite list of functions can be prepared in a very special, uniform manner called "simultaneous tight preparation." Using this and the assumption that such functions $F$ can be prepared, Section 5 prepares all $\mathcal{L}_{\mathcal{F}}^{\mathbb{Q}}$-terms by an induction over the terms. So it remains to prepare $F$.

In [13] they use the finiteness property [8, Lemma 4.12] and Weierstrass preparation to prepare all restricted analytic functions. Then $F$ is prepared by observing that there are functions $f_{+}$and $f_{-}$, analytic at the origin, such that $F(x, y)=$ $f_{+}(x, y)+(g(x) / y) f_{-}(x, g(x) / y)$, the so-called "Splitting Lemma". Preparing $f_{+}(x, y)$ and $\left.(g(x) / y)\right) f_{-}(x, g(x) / y)$ separately then enables $F(x, y)$ to be $\mathcal{L}_{\text {an }}^{\mathbb{Q}}$ prepared. The problem is that this argument does not show that $F$ is $\mathcal{L}_{\mathcal{F}}^{\mathbb{Q}}$-prepared because there seems to be no reason that $f_{+}$and $f_{-}$should be $\mathcal{F}$-analytic given that $f$ and $g$ are $\mathcal{F}$-analytic.

So the goal of Sections 6 and 7 is to give a way of preparing $F$ that only uses Weierstrass preparation. Section 6] presents the workhorse behind the preparation theorem, a local resolution of singularities procedure which performs local blowingsup and power substitutions to transform an analytic function to normal crossings. It is a slight variant of Rolin, Speissegger and Wilkie [21, Theorem 2.5], which in turn is a simple version of the local resolution procedures that have occurred in Bierstone and Milman's work, such as in [2, Theorem 4.4]. The reason for the use of power substitutions, and not just local blowings-up, is that the transformations used in the resolution procedure must be carefully chosen so as to behave well with respect to the given coordinate projection $\Pi_{n}(x, y)=x$. This enables the transformations to be unwound to prepare the given analytic function in the original coordinate system.

Section 7 prepares $F$ by combining the resolution procedure with a technique of Parusińki's involving Weierstrass preparation to find the zeros of $F$. The way these two ideas are combined is modeled after Parusiński's proof of his preparation theorem for locally blow-analytic functions [19, Theorem 7.5] but is much more elementary so that it can be seen to go through for Weierstrass systems. Thus Section 7 completes the proof of the preparation theorem for $K=\mathbb{Q}$. This is the only section which actually uses Weierstrass preparation.

For completeness, Section 8 presents an exposition of Lion and Rolin's argument that shows how the preparation theorem for $K=\mathbb{Q}$ can be used to give the preparation theorem for a general subfield $K$ of the field of exponents of $\mathcal{F}$.

We conclude the paper with Section 9, which is an appendix discussing various closure properties one might want to impose upon an analytic system of germs. We compare Weierstrass systems to Van den Dries's notion of a "convergent Weierstrass system" [22], discuss the question of whether Weierstrass preparation is closed under 
definability, and discuss closure properties related to Lion and Rolin's splitting technique and their globally subanalytic integration theorem [14. The primary purpose of the appendix is to point out the merits and deficits of our assumption that $\mathcal{F}$ is a Weierstrass system.

\section{Simultaneous tight preparation}

The goal of this section is to prove Lemma 4.4, a technical improvement on the notion of "prepared" which will be frequently used in subsequent sections. The definitions and lemmas of this section are stated and proved for $\mathcal{L}_{\mathcal{F}}^{K}$-preparation but clearly also apply to both $\mathbb{N}$ - and $\mathbb{Z}$-preparation as well.

Definition 4.1. The functions $f_{1}, \ldots, f_{m}: \mathbb{R}^{n+1} \rightarrow \mathbb{R}$ are simultaneously $\mathcal{L}_{\mathcal{F}}^{K}$ prepared on $A \subseteq \mathbb{R}^{n+1}$ if there is a common $\mathcal{L}_{\mathcal{F}}^{K}$-cylinder covering of $A$ preparing each $f_{i}, i=1, \ldots, m$, such that the $\theta(x)$ 's given by this preparation are uniform for all $i$. More precisely, there is a finite cover $\mathcal{C}$ of $A$ by $\mathcal{L}_{\mathcal{F}}^{K}$-cylinders such that for each $C \in \mathcal{C}$, if $C$ is thin, then the graph of each $\left.f_{i}\right|_{C}$ is given by an $\mathcal{L}_{\mathcal{F}}^{K}$-term in $x$, and if $C$ is fat, then there is an $\mathcal{L}_{\mathcal{F}}^{K}$-term $\theta(x)$ which is $\mathcal{F}$-analytic on $\Pi_{n}(C)$ such that for $i=1, \ldots, m$,

$$
f_{i}(x, y)=a_{i}(x)|y-\theta(x)|^{r_{i}} u_{i}(x, y-\theta(x)),
$$

on $C$, where $a_{i}, r_{i}$ and $u_{i}$ are as in Definition 3.5 .

Definition 4.2. Given $\epsilon>1$, a set $A \subseteq \mathbb{R}^{n}$ and two functions $f, g: A \rightarrow \mathbb{R}$, we say that $f$ is $\epsilon$-equivalent to $g$ on $A$, written $f \sim_{\epsilon} g$ on $A$, if for all $x \in A$,

$$
\begin{aligned}
& \epsilon^{-1} f(x) \leq g(x) \leq \epsilon f(x) \quad \text { if } \quad f(x) \geq 0 \\
& \epsilon f(x) \leq g(x) \leq \epsilon^{-1} f(x) \quad \text { if } \quad f(x)<0 .
\end{aligned}
$$

We say that $f$ is equivalent to $g$ on $A$, written $f \sim g$ on $A$, if $f \sim_{\epsilon} g$ on $A$ for some $\epsilon>1$.

Definition 4.3. Let $\epsilon>1$. Consider functions $f_{1}, \ldots, f_{m}: \mathbb{R}^{n+1} \rightarrow \mathbb{R}$ which are simultaneously $\mathcal{L}_{\mathcal{F}}^{K}$-prepared on $A \subseteq \mathbb{R}^{n+1}$; we use the notation of Definition 4.1 If we further have that either $y \sim_{\epsilon} \theta(x)$ on $C$ or else $\theta(x)$ is identically zero on $\Pi_{n}(C)$, then we say that the simultaneous preparation of the $f_{i}$ 's on $A$ is $\epsilon$-tight. We say that the simultaneous preparation of the $f_{i}$ 's is tight if it is $\epsilon$-tight for some $\epsilon>1$.

Lemma 4.4 (Simultaneous tight preparation). Suppose that $K$ is a subfield of the field of exponents of $\mathcal{F}, \epsilon>1$, and $A \subseteq \mathbb{R}^{n+1}$ is a finite union of $\mathcal{L}_{\mathcal{F}}^{K}$-cylinders. If $f_{1}, \ldots, f_{m}: \mathbb{R}^{n+1} \rightarrow \mathbb{R}$ are $\mathcal{L}_{\mathcal{F}}^{K}$-prepared on $A$, then there is a simultaneous $\mathcal{L}_{\mathcal{F}}^{K}$-preparation of $f_{1}, \ldots, f_{m}$ on $A$ which is $\epsilon$-tight.

To prove Lemma 4.4 we need two other lemmas.

Lemma 4.5. Suppose that $\theta(x)$ is an $\mathcal{L}_{\mathcal{F}}^{K}$-term, $A \subseteq \mathbb{R}^{n+1}$ is a finite union of $\mathcal{L}_{\mathcal{F}}^{K}$-cylinders and $\epsilon>1$. Then there is a finite cover $\mathcal{C}$ of $A$ by $\mathcal{L}_{\mathcal{F}}^{K}$-cylinders such that for each $C \in \mathcal{C}$ at least one of the following holds:

1. $y \sim_{\epsilon} \theta(x)$ on $C$;

2. $y-\theta(x)=a(x) u(x, y)$ on $C$, where $u(x, y)$ is an $\mathcal{L}_{\mathcal{F}}^{K}$-special unit on $C$ and a $(x)$ is an $\mathcal{L}_{\mathcal{F}}^{K}$-term;

3. $y-\theta(x)=y u(x, y)$ on $C$, where $u(x, y)$ is an $\mathcal{L}_{\mathcal{F}}^{K}$-special unit on $C$. 
Proof. We may assume that $\theta$ has constant sign on $A$. If $\theta=0$ on $A$ we are in case 3 , so assume that $\theta>0$ on $A$ (the case $\theta<0$ is similar). Fix $\delta \in(1, \epsilon)$, and let

$$
\begin{aligned}
& A_{1}:=\left\{(x, y) \in A: \epsilon^{-1} \theta(x)<y<\epsilon \theta(x)\right\}, \\
& A_{2}:=\left\{(x, y) \in A:-\epsilon \theta(x)<y<\delta^{-1} \theta(x)\right\}, \\
& A_{3}:=\{(x, y) \in A:|y|>\delta \theta(x)\} .
\end{aligned}
$$

Each of these sets is a finite union of cylinders, and they cover $A$. We claim that we are in case $i$ on $A_{i}$ for $i=1,2,3$. Indeed, we have $y \sim_{\epsilon} \theta(x)$ on $A_{1}$. On $A_{2}$ we have $y-\theta(x)=\theta(x)[y / \theta(x)-1]$, and the second term is a special unit. On $A_{3}$ we have

$$
y-\theta(x)=y[1-\theta(x) / y]
$$

and the second term a special unit.

Lemma 4.6. Suppose that $\theta_{1}(x)$ and $\theta_{2}(x)$ are $\mathcal{L}_{\mathcal{F}}^{K}$-terms and that $A \subseteq \mathbb{R}^{n+1}$ is a finite union of $\mathcal{L}_{\mathcal{F}}^{K}$-cylinders. Then there is a finite cover $\mathcal{C}$ of $A$ by $\mathcal{L}_{\mathcal{F}}^{K}$-cylinders such that for each $C \in \mathcal{C}$ at least one of the following holds:

1. $y-\theta_{2}(x)=a(x) u\left(x, y-\theta_{1}(x)\right)$ on $C$;

2. $y-\theta_{1}(x)=a(x) u\left(x, y-\theta_{2}(x)\right)$ on $C$;

3. $y-\theta_{2}(x)=\left(y-\theta_{1}(x)\right) u\left(x, y-\theta_{1}(x)\right)$ on $C$;

4. $y-\theta_{1}(x)=\left(y-\theta_{2}(x)\right) u\left(x, y-\theta_{2}(x)\right)$ on $C$;

where $a(x)$ is an $\mathcal{L}_{\mathcal{F}}^{K}$-term and $u(x, y)$ is an $\mathcal{L}_{\mathcal{F}}^{K}$-special unit.

Proof. We may assume that $\theta_{1}-\theta_{2}$ has constant sign on $A$. Since we are in both cases 3 and 4 if $\theta_{1}=\theta_{2}$, and the other two cases are symmetric, we may assume that $\theta_{1}>\theta_{2}$.

Choose constants $a$ and $b$ such that $\frac{1}{2}<a<1<b<1+a$, and consider the following sets, each of which is a finite union of cylinders:

$$
\begin{aligned}
& A_{1}:=\left\{(x, y) \in A: \theta_{1}(x)-a\left(\theta_{1}(x)-\theta_{2}(x)\right)<y<\theta_{1}(x)+a\left(\theta_{1}(x)-\theta_{2}(x)\right)\right\}, \\
& A_{2}:=\left\{(x, y) \in A: \theta_{2}(x)-a\left(\theta_{1}(x)-\theta_{2}(x)\right)<y<\theta_{2}(x)+a\left(\theta_{1}(x)-\theta_{2}(x)\right)\right\}, \\
& A_{3}:=\left\{(x, y) \in A: y<\theta_{1}(x)-b\left(\theta_{1}(x)-\theta_{2}(x)\right)\right\}, \\
& A_{4}:=\left\{(x, y) \in A: y>\theta_{2}(x)+b\left(\theta_{1}(x)-\theta_{2}(x)\right)\right\} .
\end{aligned}
$$

By the choice of $a$ and $b$, these sets cover $A$. We claim that for $i=1, \ldots, 4$, we are case $i$ on $A_{i}$. Indeed, on $A_{1}$ we have

$$
y-\theta_{2}(x)=\left(\theta_{1}(x)-\theta_{2}(x)\right)\left[1+\left(y-\theta_{1}(x)\right) /\left(\theta_{1}(x)-\theta_{2}(x)\right)\right],
$$

and the second term is a special unit. The set $A_{2}$ is similar. On $A_{3}$ we have

$$
y-\theta_{2}(x)=\left(y-\theta_{1}(x)\right)\left[1+\left(\theta_{1}(x)-\theta_{2}(x)\right) /\left(y-\theta_{1}(x)\right)\right],
$$

and the second term is a special unit. The set $A_{4}$ is similar.

Proof of Lemma 4.4. By induction on $m$. Consider $m=1$, and let $\mathcal{C}$ be a finite covering of $\mathbb{R}^{n+1}$ by cylinders such that $f_{1}$ is of a prepared form on each $C \in \mathcal{C}$. We have to show that this preparation can be made $\epsilon$-tight. Consider a fat $C \in \mathcal{C}$, and write

$$
f_{1}(x, y)=a(x)|y-\theta(x)|^{r} u(x,|y-\theta(x)|)
$$


on $C$. By Lemma 4.5 we may assume that on $C$ either $y \sim_{\epsilon} \theta(x), y-\theta(x)=$ $b(x) v(x, y)$ or $y-\theta(x)=y v(x, y)$, where $b(x)$ is a term and $v(x, y)$ is a special unit. We are done in the first case. In the second case

$$
f_{1}(x, y)=A(x) U(x, y)
$$

on $C$, where $A(x):=a(x)|b(x)|^{r}$ and $U(x, y):=|v(x, y)|^{r} u(x,|b(x) v(x, y)|)$, which is a special unit since $K$ is a subfield of the field of exponents of $\mathcal{F}$. Similarly, in the third case

$$
f_{1}(x, y)=a(x)|y|^{r} U(x, y)
$$

on $C$ for an appropriately defined special unit $U(x, y)$. Hence the preparation is $\epsilon$-tight.

Now consider $m>1$. Fix a finite cover $\mathcal{C}$ of $\mathbb{R}^{n+1}$ by cylinders such that $f_{1}$ is of an $\epsilon$-tight prepared form on each $C \in \mathcal{C}$. Consider a fat $C \in \mathcal{C}$ and write

$$
f_{1}(x, y)=a_{1}(x)\left|y-\theta_{1}(x)\right|^{r_{1}} u_{1}\left(x,\left|y-\theta_{1}(x)\right|\right),
$$

where $y \sim_{\epsilon} \theta_{1}(x)$ on $C$, provided that $\theta_{1}(x)$ is not identically zero. By applying the induction hypothesis and possibly dividing each of the cylinders of $\mathcal{C}$ into smaller cylinders, we may assume that $f_{2}, \ldots, f_{m}$ are of an $\epsilon$-tight simultaneous prepared form on $C$. Write

$$
f_{i}(x, y)=a_{i}(x)\left|y-\theta_{2}(x)\right|^{r_{i}} u_{i}\left(x,\left|y-\theta_{2}(x)\right|\right), \text { for } i=2, \ldots, m,
$$

where $y \sim_{\epsilon} \theta_{2}(x)$ on $C$, provided that $\theta_{2}(x)$ is not identically zero.

By Lemma 4.6 we may further assume, for example, that

$$
y-\theta_{1}(x)=\left(y-\theta_{2}(x)\right) v\left(x, y-\theta_{2}(x)\right)
$$

for some special unit $v$ (the other cases given by Lemma 4.6 are handled similarly). Then

$$
f_{1}(x, y)=a_{1}(x)\left|y-\theta_{2}(x)\right|^{r_{1}} U\left(x,\left|y-\theta_{2}(x)\right|\right)
$$

on $C$ for some special unit $U(x, y)$. Hence $f_{1}, \ldots, f_{m}$ have a simultaneous $\epsilon$-tight preparation.

\section{LION AND ROLIN'S INDUCTION OVER THE TERMS}

Modulo Proposition 5.1, which is proved in Sections 6 and 7, this section shows how to $\mathcal{L}_{\mathcal{F}}^{\mathbb{Q}}$-prepare all $\mathcal{L}_{\mathcal{F}}^{\mathbb{Q}}$-terms.

Proposition 5.1 (Main Proposition). Define $\varphi:\left\{(x, y, z) \in \mathbb{R}^{n+2}: z \neq 0\right\} \rightarrow$ $\mathbb{R}^{n+2}$ by

$$
\varphi(x, y, z):=(x, y / z, z),
$$

and let $A \subseteq \operatorname{dom}(\varphi)$ be a finite union of $\mathcal{L}_{\mathcal{F}}^{\mathbb{Q}}$-cylinders such that $\varphi(A)$ is bounded. If $f: \mathbb{R}^{n+2} \rightarrow \mathbb{R}$ is $\mathcal{F}$-analytic on $\operatorname{cl}(\varphi(A))$, then $f \circ \varphi$ is $\mathbb{Z}$-prepared on $A$.

Lemma 5.2. Define $\varphi: \mathbb{R}^{n+1} \rightarrow \mathbb{R}^{N+1}$ by

$$
\varphi(x, y)=\left(a(x), b(x)|y-\theta(x)|^{1 / p}\right),
$$

where $p$ is a positive integer and $\theta(x), a(x)=\left(a_{1}(x), \ldots, a_{N}(x)\right)$ and $b(x)$ are all $\mathcal{L}_{\mathcal{F}}^{\mathbb{Q}}$-terms. Let $A \subseteq \mathbb{R}^{N+1}$ be a finite union of $\mathcal{L}_{\mathcal{F}}^{\mathbb{Q}}$-cylinders. Then

(i) $\varphi^{-1}(A)$ is a finite union of $\mathcal{L}_{\mathcal{F}}^{\mathbb{Q}}$-cylinders;

(ii) if $f: \mathbb{R}^{N+1} \rightarrow \mathbb{R}$ is $\mathcal{L}_{\mathcal{F}}^{\mathbb{Q}}$-prepared on $A$, then $f \circ \varphi$ is $\mathcal{L}_{\mathcal{F}}^{\mathbb{Q}}$-prepared on $\varphi^{-1}(A)$. 
Proof. Statement (i) is clear. To show (ii), fix a finite cover $\mathcal{C}$ of $A$ by cylinders such that $f$ is of a tight prepared form on each $C \in \mathcal{C}$; we use coordinates $(X, Y)=$ $\left(X_{1}, \ldots, X_{N}, Y\right)$ in $\mathbb{R}^{N+1}$. Let $C \in \mathcal{C}$. If $C$ is thin, $f(X, Y)$ is given by a term in $X$ on $C$, so $f \circ \varphi(x, y)$ is given by a term in $x$ on $\varphi^{-1}(C)$. So we may assume that $C$ is fat and write

$$
f(X, Y)=c(X)|Y-\psi(X)|^{r} u(X, Y-\psi(X))
$$

on $C$. So

$$
\begin{array}{r}
f \circ \varphi(x, y)=c \circ a(x)|b(x)| y-\left.\theta(x)\right|^{1 / p}-\left.\psi \circ a(x)\right|^{r} \\
u\left(a(x), b(x)|y-\theta(x)|^{1 / p}-\psi \circ a(x)\right)
\end{array}
$$

on $\varphi^{-1}(C)$. We are done if $\psi=0$, so we may assume that $\psi$ is not identically zero and that $Y \sim \psi(X)$ on $C$ since the preparation of $f$ is tight. By dividing $\varphi^{-1}(C)$ into smaller cylinders we may assume that $y>\theta(x)$ on $\varphi^{-1}(C)$ (the case $y<\theta(x)$ is similar). Now, put $\alpha:=b(x)(y-\theta(x))^{1 / p}$ and $\beta:=\psi \circ a(x)$, compute

$$
\alpha-\beta=\frac{\alpha^{p}-\beta^{p}}{\sum_{i=1}^{p} \alpha^{p-i} \beta^{i-1}}=\frac{\alpha^{p}-\beta^{p}}{\alpha^{p-1}} \cdot \frac{1}{\sum_{i=1}^{p}(\beta / \alpha)^{i-1}},
$$

and note that $v(x, y-\theta(x)):=\left(\sum_{i=1}^{p}(\beta / \alpha)^{i-1}\right)^{-1}$ is a special unit since $\alpha \sim \beta$ on $\varphi^{-1}(C)$. Put $\theta^{\prime}(x)=\theta(x)+(\psi \circ a(x) / b(x))^{p}$. By (5.1)

$$
\begin{aligned}
f \circ \varphi(x, y)= & c \circ a(x)|b(x)|^{r} \cdot\left|y-\theta^{\prime}(x)\right|^{r}|y-\theta(x)|^{r(1-p) / p} \cdot v(x, y-\theta(x))^{r} \\
& \cdot u\left(a(x), b(x)\left(y-\theta^{\prime}(x)\right)(y-\theta(x))^{(1-p) / p} v(x, y-\theta(x))\right)
\end{aligned}
$$

on $\varphi^{-1}(C)$. Now apply Lemma 4.6 to $y-\theta(x)$ and $y-\theta^{\prime}(x)$ to finish preparing $f \circ \varphi$ on $\varphi^{-1}(C)$.

Lemma 5.3 (Composition Lemma). Let $A \subseteq \mathbb{R}^{n+1}$ be a finite union of $\mathcal{L}_{\mathcal{F}}^{\mathbb{Q}}$ cylinders, let $g=\left(g_{1}, \ldots, g_{m}\right): A \rightarrow[-1,1]^{m}$ be such that each $g_{i}$ is $\mathcal{L}_{\mathcal{F}}^{\mathbb{Q}}$-prepared on $A$, and let $f:[-1,1]^{n} \rightarrow \mathbb{R}$ be $\mathcal{F}$-analytic. Then $f \circ g$ is $\mathcal{L}_{\mathcal{F}}^{\mathbb{Q}}$-prepared on $A$.

Proof. By Lemma 4.4, the $g_{i}$ 's are simultaneously prepared on $A$. It follows that $f \circ g$ is an $\mathcal{L}_{\mathcal{F}}^{\mathbb{Q}}$-special function: for example, if $m=1$ and

$$
g(x, y)=a(x)|y-\theta(x)|^{q / p} u\left(b(x), c(x)|y-\theta(x)|^{1 / p}, d(x)|y-\theta(x)|^{-1 / p}\right)
$$

on a cylinder $C$, where $u$ is an $\mathcal{F}$-analytic unit, $q$ and $p$ are positive integers, and $a(x), \theta(x), b(x)=\left(b_{1}(x), \ldots, b_{M}(x)\right), c(x)$ and $d(x)$ are all terms, then

$$
f \circ g(x, y)=F\left(b(x), c(x)|y-\theta(x)|^{1 / p}, d(x)|y-\theta(x)|^{-1 / p}, a(x)^{1 / q}|y-\theta(x)|^{1 / p}\right)
$$

for $F\left(x_{1}, \ldots, x_{M+3}\right):=f\left(x_{M+3}^{q} u\left(x_{1}, \ldots, x_{M+2}\right)\right)$, assuming as we may that $a(x)>$ 0 .

By Lemma 3.4 there is a finite cover $\mathcal{C}$ of $A$ by cylinders such that for each $C \in \mathcal{C}$, $f \circ g(x)=F \circ \varphi(x, y)$ on $C$, where $F: \mathbb{R}^{N+2} \rightarrow \mathbb{R}$ is $\mathcal{F}$-analytic on $\operatorname{cl}(\varphi(C))$ and $\varphi: C \rightarrow \mathbb{R}^{N+2}$ is a bounded function of the form

$$
\varphi(x, y)=\left(a(x), b(x)|y-\theta(x)|^{1 / p}, c(x)|y-\theta(x)|^{-1 / p}\right)
$$

for a positive integer $p$ and terms $a(x)=\left(a_{1}(x), \ldots, a_{N}(x)\right), b(x), c(x)$ and $\theta(x)$. Consider new variables $X=\left(X_{1}, \ldots, X_{N}\right), Y$ and $Z$, and view the map $\varphi$ as the 
composition $\varphi=\varphi_{2} \circ \varphi_{1}$, where $\varphi_{1}: C \rightarrow \mathbb{R}^{N+2}$ and $\varphi_{2}:\left\{(X, Y, Z) \in \mathbb{R}^{N+2}: Z \neq\right.$ $0\} \rightarrow \mathbb{R}^{N+2}$ are given by

$$
\begin{aligned}
\varphi_{1}(x, y) & =\left(a(x), b(x) c(x), b(x)|y-\theta(x)|^{1 / p}\right), \\
\varphi_{2}(X, Y, Z) & =(X, Z, Y / Z) .
\end{aligned}
$$

Let $U$ be a bounded neighborhood of $\operatorname{cl}(\varphi(C))$ on which $F$ is $\mathcal{F}$-analytic; we may take $U$ to be a finite union of open boxes. Now, $\varphi_{2}^{-1}(U)$ is a finite union of cylinders, and its image under $\varphi_{2}$, namely $U$, is bounded. So by the Main Proposition, $F \circ \varphi_{2}$ is $\mathbb{Z}$-prepared on $\varphi_{2}^{-1}(U)$. Also, $\varphi_{1}(C) \subseteq \varphi_{2}^{-1}(U)$, so $C=\varphi_{1}^{-1}\left(\varphi_{2}^{-1}(U)\right)$. Therefore by Lemma [5.2, $F \circ \varphi_{2} \circ \varphi_{1}$ is prepared on $C$.

Proof of the Main Theorem for $K=\mathbb{Q}$. We show that every $\mathcal{L}_{\mathcal{F}}^{\mathbb{Q}}$-term is $\mathcal{L}_{\mathcal{F}}^{\mathbb{Q}}$ prepared. This follows directly from the following claims.

Claim 1. If $f, g: \mathbb{R}^{n+1} \rightarrow \mathbb{R}$ are $\mathcal{L}_{\mathcal{F}}^{\mathbb{Q}}$-prepared, then so are $f \cdot g, f / g$ and $f^{r}$ for all $r \in \mathbb{Q}$.

Claim 2. If $f_{1}, f_{2}: \mathbb{R}^{n+1} \rightarrow \mathbb{R}$ are $\mathcal{L}_{\mathcal{F}}^{\mathbb{Q}}$-prepared, then so is $f_{1}+f_{2}$.

Claim 3. If $g=\left(g_{1}, \ldots, g_{m}\right): \mathbb{R}^{n+1} \rightarrow \mathbb{R}^{m}$ is such that each $g_{i}$ is $\mathcal{L}_{\mathcal{F}}^{\mathbb{Q}}$-prepared, and $f: \mathbb{R}^{m} \rightarrow \mathbb{R}$ is a restricted $\mathcal{F}$-analytic function, then $f \circ g$ is $\mathcal{L}_{\mathcal{F}}^{\mathbb{Q}}$-prepared.

Claim 1 is obvious by simultaneous preparation, so we prove Claims 2 and 3 .

Proof of Claim 2. Fix a finite cover $\mathcal{C}$ of $\mathbb{R}^{n+1}$ by cylinders such that $f_{1}$ and $f_{2}$ are of a simultaneously prepared form on each $C \in \mathcal{C}$. If $C \in \mathcal{C}$ is thin, $\left.\left(f_{1}+f_{2}\right)\right|_{C}$ is given by a term in $x$. So it suffices to consider a fat $C \in \mathcal{C}$ and write

$$
f_{i}(x, y)=a_{i}(x)|y-\theta(x)|^{r_{i}} u_{i}(x,|y-\theta(x)|)
$$

on $C$ for $i=1$,2. Fix $0<\epsilon<M$ such that $\epsilon<\left|u_{i}(x,|y-\theta(x)|)\right|<M$ on $C$, and cover $C$ with the following three sets, each of which is a finite union of cylinders:

$$
\begin{aligned}
C_{f_{1} \sim f_{2}}:=\left\{(x, y) \in C: \frac{\epsilon}{2 M} \leq\left|\frac{a_{1}(x)}{a_{2}(x)}\right||y-\theta(x)|^{r_{1}-r_{2}} \leq \frac{2 M}{\epsilon}\right\}, \\
C_{f_{1} \gg f_{2}}:=\left\{(x, y) \in C:\left|\frac{a_{1}(x)}{a_{2}(x)}\right||y-\theta(x)|^{r_{1}-r_{2}} \geq \frac{2 M}{\epsilon}\right\}, \\
C_{f_{1} \ll f_{2}}:=\left\{(x, y) \in C:\left|\frac{a_{1}(x)}{a_{2}(x)}\right||y-\theta(x)|^{r_{1}-r_{2}} \leq \frac{\epsilon}{2 M}\right\} .
\end{aligned}
$$

On $C_{f_{1} \sim f_{2}}, f_{1}+f_{2}=f_{1}\left(1+f_{2} / f_{1}\right)$ and $f_{2} / f_{1}$ is prepared and bounded. By applying Lemma 5.3 to compose $f_{2} / f_{1}$ with the function $t \mapsto 1+t$, we see that $1+f_{2} / f_{1}$ is prepared, so $f_{1}\left(1+f_{2} / f_{1}\right)$ is too.

On $C_{f_{1} \gg f_{2}}, 1+f_{2} / f_{1}$ is a special unit, so $f_{1}+f_{2}=f_{1}\left(1+f_{2} / f_{1}\right)$ is prepared. On $C_{f_{1} \ll f_{2}}, f_{1} / f_{2}+1$ is a special unit, so $f_{1}+f_{2}=f_{2}\left(f_{1} / f_{2}+1\right)$ is prepared.

Proof of Claim 3. Claim 2 shows that

$$
\left\{(x, y) \in \mathbb{R}^{n+1}:-1 \leq g_{i}(x, y) \leq 1 \text { for } i=1, \ldots, m\right\}
$$

is a finite union of cylinders, because by preparing each function $1+g_{i}$ and $1-g_{i}$, we may obtain a finite cover $\mathcal{C}$ of $\mathbb{R}^{n+1}$ by cylinders such that for all $C \in \mathcal{C}$ and 
$i=1, \ldots, m, 1+g_{i}$ and $1-g_{i}$ have constant sign on $C$. Therefore for each $C \in \mathcal{C}$, either $f \circ g=0$ on $C$ or $\left|g_{1}\right|, \ldots,\left|g_{m}\right| \leq 1$ on $C$. In the latter case apply Lemma 5.3 .

\section{Transforming to NORMAL CROSSINGS AND PREPARING FUNCTIONS IN $\mathcal{F}$}

Definitions 6.1. For $r=\left(r_{1}, \ldots, r_{n}\right)$ and $s=\left(s_{1}, \ldots, s_{n}\right)$ in $\mathbb{R}^{n}$ we write $r<s$ if $r_{1}<s_{1}, \ldots, r_{n}<s_{n}$ and write $r \leq s$ if $r_{1} \leq s_{1}, \ldots, r_{n} \leq s_{n}$. In particular for $\alpha, \beta \in \mathbb{N}^{n}, \alpha \leq \beta$ if and only if $x^{\alpha}$ divides $x^{\beta}$.

For $r=\left(r_{1}, \ldots, r_{n}\right) \in(0, \infty)^{n}$ let

$$
\mathcal{B}_{r}:=\left\{x \in \mathbb{C}^{n}:\left|x_{i}\right| \leq r_{i} \text { for } i=1, \ldots, n\right\}
$$

and $B_{r}:=\mathcal{B}_{r} \cap \mathbb{R}^{n}$. Let $\mathcal{F}_{n, r}$ denote the set of all functions from $\mathcal{B}_{r}$ into $\mathbb{C}$ which extend to a holomorphic function in a neighborhood of $\mathcal{B}_{r}$ and whose restriction to $B_{r}$ is $\mathcal{F}$-analytic.

Call $u \in \mathcal{F}_{n, r}$ a unit if $u(x) \neq 0$ for all $x \in \mathcal{B}_{r}$. A function $f \in \mathcal{F}_{n, r}$ is normal crossings if $f(x)=x^{\alpha} u(x)$ for some $\alpha \in \mathbb{N}^{n}$ and unit $u \in \mathcal{F}_{n, r}$. We also say that a germ in $\mathcal{F}_{n}$ is normal crossings if it has a normal crossings representative in $\mathcal{F}_{n, r}$ for some $r$. A function $f: \mathbb{R}^{n} \rightarrow \mathbb{R}$ is $\mathcal{F}$-normal crossings on $B_{r}$ if $\left.f\right|_{B_{r}}$ extends to a function in $\mathcal{F}_{n, r}$ which is normal crossings.

Remark 6.2. In real analytic geometry one speaks of an analytic function $f$ being "locally normal crossings"; this is a diffeomorphically invariant definition meaning that every point in the domain of $f$ admits a local system of coordinates such that $f$ is normal crossings in those coordinates. In our proof we do not have use for this definition because the notion of "preparation" is not invariant under arbitrary diffeomorphisms.

Let $1<m \leq n$, let $U \subseteq \mathbb{R}^{n}$ be an open neighborhood of the origin, let $C=\{x \in$ $\left.U: \Pi_{m}(x)=0\right\}$, let $\mathbb{P}^{m-1}(\mathbb{R})$ denote the $(m-1)$-dimensional real projective space of all lines through the origin in $\mathbb{R}^{m}$, and finally let

$$
\begin{aligned}
U^{\prime} & :=\left\{(x, \xi) \in U \times \mathbb{P}^{m-1}(\mathbb{R}): \Pi_{m}(x) \in \xi\right\} \\
& =\left\{(x, \xi) \in U \times \mathbb{P}^{m-1}(\mathbb{R}): x_{i} \xi_{j}=x_{j} \xi_{i} \text { for } i, j=1, \ldots, m\right\},
\end{aligned}
$$

where $\left[\xi_{1}: \cdots: \xi_{m}\right]$ are homogeneous coordinates for $\xi$. Then the projection map $\Pi: U^{\prime} \rightarrow U$ given by $\Pi(x, \xi)=x$ is called the blowing-up of $U$ with center $C$. More generally, if $\varphi: U \rightarrow V$ is an $\mathcal{F}$-analytic diffeomorphism (so $M:=\varphi(C)$ is a closed $\mathcal{F}$-analytic submanifold of $V$ ), then the composition $\psi:=\varphi \circ \Pi: U^{\prime} \rightarrow V$ is called the blowing-up of $V$ with center $M$.

The exceptional divisor of the blowing-up $\psi: U^{\prime} \rightarrow V$ is the inverse image of its center, $E:=\psi^{-1}(M)$. Observe that the restriction of $\psi$ to $U^{\prime} \backslash E$ is an $\mathcal{F}$ analytic diffeomorphism onto its image, and that $\psi^{-1}(a)=\left\{\varphi^{-1}(a)\right\} \times \mathbb{P}^{m-1}(\mathbb{R})$ for all $a \in M$. Using these observations, it is an easy exercise to show that for any $a \in U$ and any neighborhood $W$ of $\psi^{-1}(a), \psi(W)$ is a neighborhood of $a$. The restriction of $\psi$ to any such open neighborhood $W$ of $\psi^{-1}(a)$, for $a \in M$, is called a "local" blowing-up of $V$ "at $a$ " with center $M$.

Given a function $f(x, y)$ analytic in a neighborhood of the origin, by Bierstone and Milman [2, Theorem 4.4] there is a finite collection $T$ of analytic maps which 
are compositions of local blowings-up with smooth analytic centers such that for each $\mu \in T, f \circ \mu$ is locally normal crossings, and such that for each $\mu \in T$ there is a compact set $K_{\mu} \subseteq \operatorname{dom}(\mu)$ such that $\bigcup_{\mu \in T} \mu\left(K_{\mu}\right)$ is a neighborhood of the origin. This section presents a simple variant of Bierstone and Milman's procedure due to Speissegger which is particularly well suited to proving the preparation theorem. The source of the differences between this procedure and that of Bierstone and Milman's is that we need the transformations in $T$ to behave well with respect to the given coordinate projection $\Pi_{n}(x, y)=x$ so that the resolution procedure can be unwound to prepare $f$ back in its original coordinates. To achieve this we shall restrict ourselves to local blowings-up which have a center $C \subseteq \mathbb{R}^{n+1}$ constructed from a smooth center $C^{\prime} \subseteq \mathbb{R}^{n}$ in one of the following two ways: either $C=C^{\prime} \times \mathbb{R}$, or else $C$ is the graph of some $\mathcal{F}$-analytic function $\theta: C^{\prime} \rightarrow \mathbb{R}$ (i.e., the lifting of $C^{\prime}$ via a smooth section $\theta$ of $\Pi_{n}: \mathbb{R}^{n+1} \rightarrow \mathbb{R}^{n}$ ). As a result, we shall find it necessary to also use local power substitutions in our procedure. Moreover, even though there is no real advantage or disadvantage in regards to proving the preparation theorem, it simplifies the presentation to inductively require that the center $C^{\prime}$ is constructed from a smooth center $C^{\prime \prime} \subseteq \mathbb{R}^{n-1}$ in the same fashion, and so forth. It also simplifies the presentation, and is in fact sufficient because of the use of power substitutions, to only consider local blowings-up with centers of codimension 2. The upshot is that we shall consider transformations which are compositions of finite sequences of " $\mathcal{F}$-admissible transformations" as defined below.

Definition 6.3. A function $\mu \in\left(\mathcal{F}_{n, r}\right)^{n}$ is an $\mathcal{F}$-admissible transformation in $x$ if it is one of the following three types of maps:

(i) translation by a function: for $1 \leq i \leq n$ and $\theta \in \mathcal{F}_{i-1, s}$ such that $\theta(0)=0$, where $s=\left(r_{1}, \ldots, r_{i-1}\right)$,

$$
t_{\theta}(x)=\left(x_{1}, \ldots, x_{i-1}, x_{i}+\theta\left(x_{1}, \ldots, x_{i-1}\right), x_{i+1}, \ldots, x_{n}\right) ;
$$

(ii) power substitution: for integers $k \geq 1,1 \leq i<n$ and $\sigma \in\{-1,1\}$,

$$
p_{i, \sigma}^{k}(x)=\left(x_{1}, \ldots, x_{i-1}, \sigma x_{i}^{k}, x_{i+1}, \ldots, x_{n}\right) ;
$$

(iii) blow-up substitution: for $\lambda \in \mathbb{R} \cup\{\infty\}$ and $1 \leq i<j \leq n$,

$$
b_{\lambda}^{i, j}(x):= \begin{cases}\left(x_{1}, \ldots, x_{j-1}, x_{i}\left(x_{j}+\lambda\right), x_{j+1}, \ldots, x_{n}\right), & \text { if } \lambda \in \mathbb{R} \\ \left(x_{1}, \ldots, x_{i-1}, x_{i} x_{j}, x_{i+1}, \ldots, x_{n}\right), & \text { if } \lambda=\infty .\end{cases}
$$

If $\mu(x)=\left(\nu\left(x_{1}, \ldots, x_{i}\right), x_{i+1}, \ldots, x_{n}\right)$ for some $\mathcal{F}$-admissible transformation $\nu$ in $\left(x_{1}, \ldots, x_{i}\right)$, we shall also say that $\mu$ is an " $\mathcal{F}$-admissible transformation in $\left(x_{1}, \ldots, x_{i}\right)$.

The set of maps $\left\{b_{\lambda}^{i, j}: \lambda \in \mathbb{R} \cup\{\infty\}\right\}$ is a collection of (complexifications of) local charts for a local blowing-up of $\mathbb{R}^{n}$ at the origin with center $\left\{x \in \mathbb{R}^{n}: x_{i}=x_{j}=0\right\}$, in the following sense. Let $U \subseteq \mathbb{R}^{n}$ be a neighborhood of the origin, let

$$
\begin{aligned}
U^{\prime} & :=\left\{(x, \xi) \in U \times \mathbb{P}(\mathbb{R}):\left(x_{i}, x_{j}\right) \in \xi\right\} \\
& =\left\{(x, \xi) \in U \times \mathbb{P}(\mathbb{R}): x_{i} \xi_{j}=x_{j} \xi_{i}\right\},
\end{aligned}
$$

where $\left[\xi_{i}: \xi_{j}\right]$ are homogenous coordinates for $\xi$, and let $\Pi: U^{\prime} \rightarrow U$ be the blowing-up $\Pi(x, \xi)=x$. Note that $\Pi^{-1}(0)=\{0\} \times \mathbb{P}(\mathbb{R})=\{(0,[1: \lambda]): \lambda \in$ $\mathbb{R}\} \cup\{(0,[0: 1])\}$. We can coordinatize a neighborhood of $(0,[1: \lambda])$ in $U^{\prime}$ by letting $x_{k}$ vary near 0 for all $k \neq j$, letting $\xi_{j}$ vary near $\lambda$, setting $\xi_{i}=1$ and determining $x_{j}$ by the relation $x_{j}=x_{i} \xi_{j}$. Changing notation by replacing $\xi_{j}$ with 
$x_{j}$ and translating by $\lambda$, this means that the image of the chart $\widetilde{b}_{\lambda}^{i, j}: U_{\lambda} \rightarrow U^{\prime}$ given by $\widetilde{b}_{\lambda}^{i, j}(x)=\left(b_{\lambda}^{i, j}(x),\left[1: x_{j}+\lambda\right]\right)$ is a neighborhood of $(0,[1: \lambda])$, where $U_{\lambda}$ is a sufficiently small neighborhood of the origin in $\mathbb{R}^{n}$. Similarly, the image of the local chart $\widetilde{b}_{\infty}^{i, j}: U_{\infty} \rightarrow U^{\prime}$ given by $\widetilde{b}_{\infty}^{i, j}(x)=\left(b_{\infty}^{i, j}(x),\left[x_{i}: 1\right]\right)$ is a neighborhood of $(0,[0: 1])$ for any small neighborhood $U_{\infty}$ of the origin in $\mathbb{R}^{n}$. By the compactness of $\mathbb{P}(\mathbb{R})$ there exists a finite set $\Lambda \subseteq \mathbb{R} \cup\{\infty\}$ such that $\bigcup_{\lambda \in \Lambda} \widetilde{b}_{\lambda}^{i, j}\left(U_{\lambda}\right)$ is a neighborhood of $\Pi^{-1}(0)$ in $U^{\prime}$. Therefore $\bigcup_{\lambda \in \Lambda} b_{\lambda}^{i, j}\left(U_{\lambda}\right)$ is a neighborhood of the origin in $\mathbb{R}^{n}$, since $b_{\lambda}^{i, j}=\Pi \circ \widetilde{b}_{\lambda}^{i, j}$ for each $\lambda$.

We shall also apply the terminology and notation of Definition 6.3 to the corresponding germs of functions at the origin. The notation $t_{\theta}, p_{i, \sigma}^{k}$ and $b_{\lambda}^{i, j}$ is then well defined. But when working with actual functions, the notation $t_{\theta}, p_{i, \sigma}^{k}$ and $b_{\lambda}^{i, j}$ is somewhat deficient because the same notation may be applied to different functions with different domains but the same germ at 0 . So when working with actual functions we will always assume that some domain of polyradius $\epsilon\left(t_{\theta}\right), \epsilon\left(p_{i, \sigma}^{k}\right)$ or $\epsilon\left(b_{\lambda}^{i, j}\right)$ has been chosen.

Since $t_{\theta}$ is a homeomorphism taking 0 to 0 , its range over the reals, $t_{\theta}\left(B_{\epsilon\left(t_{\theta}\right)}\right)$, is a neighborhood of the origin. Similarly, the union of the ranges over the reals of $p_{i,-1}^{k}$ and $p_{i, 1}^{k}$ is a neighborhood of the origin (and both functions are necessary when $k$ is even). Finally, given the collection $\left\{b_{\lambda}^{i, j}: \lambda \in \mathbb{R} \cup\{\infty\}\right\}$ with any choice of polyradii $\epsilon\left(b_{\lambda}^{i, j}\right)$ for $\lambda \in \mathbb{R} \cup\{\infty\}$, by the preceding discussion there exists a finite $\Lambda \subseteq \mathbb{R} \cup\{\infty\}$ such that $\bigcup_{\lambda \in \Lambda} b_{\lambda}^{i, j}\left(B_{\epsilon\left(b_{\lambda}^{i, j}\right)}\right)$ is a neighborhood of the origin. The choice of $\Lambda$ of course depends on the choice of the $\epsilon\left(b_{\lambda}^{i, j}\right)$ 's. Because of these observations it is natural not to study just single $\mathcal{F}$-admissible transformations, but rather their "families", as defined below.

Definition 6.4. Given (the germ at 0 of) an $\mathcal{F}$-admissible transformation $\mu$ in $x$, define the family of $\mu$ as the set $\left\{t_{\theta}\right\}$ if $\mu=t_{\theta}$, the set $\left\{p_{i, 1}^{k}, p_{i,-1}^{k}\right\}$ if $\mu=p_{i, \sigma}^{k}$ for some $\sigma \in\{1,-1\}$, and the set $\left\{b_{\lambda}^{i, j}: \lambda \in \mathbb{R} \cup\{\infty\}\right\}$ if $\mu=b_{\lambda}^{i, j}$ for some $\lambda \in \mathbb{R} \cup\{\infty\}$.

Definition 6.5. Given a family $T$ of an $\mathcal{F}$-admissible transformation in $x$, define the center of $T, \operatorname{Cen}(T)$, as follows:

(i) $\operatorname{Cen}(T)=\emptyset$ if $T=\left\{t_{\theta}\right\}$;

(ii) $\operatorname{Cen}(T)=\left\{x \in \mathbb{R}^{n}: x_{i}=0\right\}$ if $T=\left\{p_{i, 1}^{k}, p_{i,-1}^{k}\right\}$;

(iii) $\operatorname{Cen}(T)=\left\{x \in \mathbb{R}^{n}: x_{i}=x_{j}=0\right\}$ if $T=\left\{b_{\lambda}^{i, j}: \lambda \in \mathbb{R} \cup\{\infty\}\right\}$.

For any $\mu \in T, \operatorname{Cen}(\mu):=\operatorname{Cen}(T)$.

Given an $\mathcal{F}$-admissible transformation $\mu$ in $x$, define the exceptional divisor of $\mu, \operatorname{Exc}(\mu)$, as follows:

(i) $\operatorname{Exc}\left(t_{\theta}\right)=\emptyset$;

(ii) $\operatorname{Exc}\left(p_{i, \sigma}^{k}\right)=\left\{x \in \mathbb{R}^{n}: x_{i}=0\right\}$;

(iii) $\operatorname{Exc}\left(b_{\lambda}^{i, j}\right)= \begin{cases}\left\{x \in \mathbb{R}^{n}: x_{i}=0\right\}, & \text { if } \lambda \in \mathbb{R}, \\ \left\{x \in \mathbb{R}^{n}: x_{j}=0\right\}, & \text { if } \lambda=\infty .\end{cases}$

If $T$ is a family of an $\mathcal{F}$-admissible transformation, then for each $\mu \in T, \mathcal{B}_{\epsilon(\mu)} \cap$ $\operatorname{Exc}(\mu)=\mu^{-1}(\operatorname{Cen}(T))$ and the restriction of $\mu$ to $\mathcal{B}_{\epsilon(\mu)} \backslash \operatorname{Exc}(\mu)$ is a diffeomorphism onto its image. 
For $\mathcal{F}$-admissible transformations to be useful, we need to see how the notions of "cylinder" and "preparation" behave under these transformations.

Lemma 6.6. Let $C \subseteq \mathbb{R}^{n+1}$ be an $\mathcal{L}_{\mathcal{F}}^{\mathbb{Q}}$-cylinder, and let $\mu$ be an $\mathcal{F}$-admissible transformation in $(x, y)$ which is not of the form $b_{\infty}^{i, n+1}, 1 \leq i \leq n$. Then

(i) the set $\mu(C)$ is a finite union of $\mathcal{L}_{\mathcal{F}}^{\mathbb{Q}}$-cylinders;

(ii) if $f: \mathbb{R}^{n+1} \rightarrow \mathbb{R}$ is such that $f \circ \mu$ is $\mathcal{L}_{\mathcal{F}}^{\mathbb{Q}}$-prepared on $C$, then $f$ is $\mathcal{L}_{\mathcal{F}}^{\mathbb{Q}}$-prepared on $\mu(C) \backslash \operatorname{Cen}(\mu)$; the same holds for both $\mathbb{N}$-preparation and $\mathbb{Z}$-preparation.

Lemma 6.7. Let $r \in(0, \infty)^{n+1}$ and $1 \leq i \leq n$.

(i) The set $b_{\infty}^{i, n+1}\left(B_{r}\right)$ is a finite union of $\mathcal{L}_{\mathcal{F}}^{\mathbb{Q}}$-cylinders.

(ii) Suppose that $f: \mathbb{R}^{n+1} \rightarrow \mathbb{R}$ is such that $f \circ b_{\infty}^{i, n+1}$ is $\mathcal{F}$-normal crossings on $B_{r}$, say of the form $f \circ b_{\infty}^{i, n+1}(x, y)=x^{\alpha} y^{d} u(x, y)$ for some unit $u \in \mathcal{F}_{n+1, r}$. Then $f$ is $\mathbb{Z}$-prepared on $b_{\infty}^{i, n+1}\left(B_{r}\right) \backslash \operatorname{Cen}\left(b_{\infty}^{i, n+1}\right)$. If we further have that $d \geq \alpha_{i}$, then $f$ is $\mathbb{N}$-prepared on $b_{\infty}^{i, n+1}\left(B_{r}\right) \backslash \operatorname{Cen}\left(b_{\infty}^{i, n+1}\right)$.

We leave most of the verification of Lemmas 6.6 and 6.7 to the reader. But it is instructive to show a little bit of their proofs to see why Lemma 6.7 deals with a box $B_{r}$ and a function $f \circ \mu$ which is normal crossings instead of a general cylinder $C$ and function $f \circ \mu$ which is prepared. Consider an $\mathcal{L}_{\mathcal{F}}^{\mathbb{Q}}$-cylinder $C \subseteq \mathbb{R}^{n+1}$, say

$$
C=\{(x, y) \in B \times \mathbb{R}: s(x)<y<t(x)\}
$$

with $s(x)<t(x)$ on $B$. Let $\mu=b_{\lambda}^{i, n+1}$ for some $\lambda \in \mathbb{R} \cup\{\infty\}$, and suppose that $f \circ \mu$ is of an $\mathbb{N}$-prepared form on $C$, say

$$
f \circ \mu(x, y)=a(x)(y-\theta(x))^{d} u(x, y-\theta(x)) .
$$

First suppose that $\lambda \in \mathbb{R}$. Then $\mu^{-1}(x, y)=\left(x, y / x_{i}-\lambda\right)$ for $x_{i} \neq 0$. So $\mu(C)$ is the union of the cylinder $\mu(C) \cap \operatorname{Cen}(\mu)=\left\{(x, y) \in B \times \mathbb{R}: x_{i}=0, y=0\right\}$ and the cylinders

$$
\begin{aligned}
\mu(C) \backslash \operatorname{Cen}(\mu)= & \left\{(x, y) \in B \times \mathbb{R}: x_{i}>0, x_{i}(s(x)+\lambda)<y<x_{i}(t(x)+\lambda)\right\} \\
& \cup\left\{(x, y) \in B \times \mathbb{R}: x_{i}<0, x_{i}(t(x)+\lambda)<y<x_{i}(s(x)+\lambda)\right\} .
\end{aligned}
$$

Applying $\mu^{-1}$ to (6.1) shows that

$$
f(x, y)=\frac{a(x)}{x_{i}^{d}}\left(y-x_{i}(\lambda+\theta(x))\right)^{d} u\left(x, \frac{y-x_{i}(\lambda+\theta(x))}{x_{i}}\right)
$$

on $\mu(C) \backslash \operatorname{Cen}(\mu)$, which is $\mathbb{N}$-prepared.

Now suppose that $\lambda=\infty$. Then $\mu^{-1}(x, y)=\left(x_{1}, \ldots, x_{i} / y, \ldots, x_{n}, y\right)$ for $y \neq 0$. Therefore $\mu(C)$ is the union of the two sets $(B \times \mathbb{R}) \cap \operatorname{Cen}(\mu)=\{(x, y) \in B \times \mathbb{R}$ : $\left.x_{i}=y=0\right\}$ and $\mu(C) \backslash \operatorname{Cen}(\mu)$, which is given by

$$
\left\{(x, y) \in \mathbb{R}^{n+1}: \begin{array}{l}
y \neq 0,\left(x_{1}, \ldots, x_{i} / y, \ldots, x_{n}\right) \in B \\
s\left(x_{1}, \ldots, x_{i} / y, \ldots, x_{n}\right)<y<t\left(x_{1}, \ldots, x_{i} / y, \ldots, x_{n}\right)
\end{array}\right\} .
$$

Because $B$ could be a rather complicated quantifier free definable set, and $s(x)$ and $t(x)$ could be rather complicated $\mathcal{L}_{\mathcal{F}}^{\mathbb{Q}}$-terms, it is not at all clear that $\mu(C) \backslash \operatorname{Cen}(\mu)$ is a finite union of $\mathcal{L}_{\mathcal{F}}^{\mathbb{Q}}$-cylinders, as it in fact must be by the preparation theorem we are trying to prove. Similarly, it is not at all clear that $f$ is prepared on $\mu(C) \backslash \operatorname{Cen}(\mu)$, because it is the product of $a\left(x_{1}, \ldots, x_{i} / y, \ldots, x_{n}\right),\left(y-\theta\left(x_{1}, \ldots, x_{i} /\right.\right.$ $\left.\left.y, \ldots, x_{n}\right)\right)^{d}$ and a unit, and both $a(x)$ and $\theta(x)$ could be rather complicated $\mathcal{L}_{\mathcal{F}^{-}}^{\mathbb{Q}}$ terms. But in the special case that $C=B_{r}$ and $f \circ \mu$ is $\mathcal{F}$-normal crossings, say of 
the form $f \circ \mu(x, y)=x^{\alpha} y^{d} u(x, y)$ for an analytic unit $u, \mu\left(B_{r}\right)$ is indeed a finite union of cylinders and

$$
f(x, y)=x^{\alpha} y^{d-\alpha_{i}} u\left(x_{1}, \ldots, x_{i} / y, \ldots, x_{n}, y\right)
$$

on $\mu\left(B_{r}\right) \backslash \operatorname{Cen}(\mu)$, which is $\mathbb{Z}$-prepared. If $d \geq \alpha_{i}, f$ is $\mathbb{N}$-prepared.

Definition 6.8. Given $\mathcal{F}$-admissible transformations $\mu^{j} \in\left(\mathcal{F}_{n, \epsilon\left(\mu^{j}\right)}\right)^{n}$ for $j=$ $1, \ldots, m$, we call the sequence $\mu=\left\langle\mu^{1}, \ldots, \mu^{m}\right\rangle$ an $\mathcal{F}$-transformation sequence in $x$ if $\mu^{j+1} \circ \cdots \circ \mu^{m}\left(\mathcal{B}_{\epsilon\left(\mu^{m}\right)}\right) \subseteq \mathcal{B}_{\epsilon\left(\mu^{j}\right)}$ for $j=1, \ldots, m-1$. We identify $\mu$ with the map $\mu^{1} \circ \cdots \circ \mu^{m} \in\left(\mathcal{F}_{n, \epsilon\left(\mu^{m}\right)}\right)^{n}$. We say that $\mu$ is an " $\mathcal{F}$-transformation sequence in $\left(x_{1}, \ldots, x_{i}\right)$ ", where $1 \leq i \leq n$, if each $\mu^{j}$ is an $\mathcal{F}$-transformation in $\left(x_{1}, \ldots, x_{i}\right)$.

Since an $\mathcal{F}$-admissible transformation is a continuous function taking 0 to 0 , the notion of an $\mathcal{F}$-transformation sequence can also be applied to the corresponding germs at 0 . And conversely, given any $\mathcal{F}$-transformation sequence $\mu$ of germs $\mu^{1}, \ldots \mu^{m}$, polyradii $\epsilon\left(\mu^{j}\right)$ can be chosen so that the functions $\mu^{j}: \mathcal{B}_{\epsilon\left(\mu^{j}\right)} \rightarrow \mathbb{C}^{n}$, for $j=1, \ldots, m$, representing these germs form an $\mathcal{F}$-transformation sequence, and such that the range of the map $\mu$ lies in any given neighborhood of the origin.

Definition 6.9. Given a set $T$ of $\mathcal{F}$-transformation sequences, define the height of $T$ by $\operatorname{ht}(T):=\sup \left\{m \in \mathbb{N}:\left\langle\mu^{1}, \ldots, \mu^{m}\right\rangle \in T\right\} \in \mathbb{N} \cup\{\infty\}$. The set $T$ is "in $\left(x_{1}, \ldots, x_{i}\right)$ " if each $\mu \in T$ is in $\left(x_{1}, \ldots, x_{i}\right)$.

Given some $0 \neq f \in \mathcal{F}_{n, r}$, we are interested in constructing a finite set $S$ of $\mathcal{F}$-transformation sequences such that for each $\mu \in S, f \circ \mu$ is normal crossings, and such that the ranges over the reals of the maps in $S$ cover a neighborhood of the origin contained in $B_{r}$. To do so we will construct a set $T$ of $\mathcal{F}$-transformation sequences such that $\operatorname{ht}(T)<\infty$ and such that for each $\mu=\left\langle\mu^{1}, \ldots, \mu^{m}\right\rangle \in T$ and $j=1, \ldots, m$,

(i) $\left\langle\mu^{1}, \ldots, \mu^{j-1}\right\rangle \notin T$;

(ii) $\left\{\nu^{j}:\left\langle\mu^{1}, \ldots, \mu^{j-1}\right\rangle \subseteq \nu \in T\right\}$ is exactly the family of $\mu^{j}$, where $\subseteq$ denotes sequence inclusion (i.e., the initial segment relation).

The desired $S$ will then be chosen as a finite subset of $T$.

Note that $T^{\prime}:=\{\nu: \nu \subseteq \mu$ for some $\mu \in T\}$ is a tree under the inclusion ordering and that $T^{\prime}$ always branches according to entire transformation families. The set $T$ is the set of maximal members of $T^{\prime}$ and can be identified with the set of branches of $T^{\prime}$. Since $T$ and $T^{\prime}$ uniquely determine one another, we abuse terminology and consider $T$ to be a tree.

Definition 6.10. A full $\mathcal{F}$-transformation tree is a set $T$ of $\mathcal{F}$-transformation sequences of finite height satisfying (i) and (ii) above for all $\left\langle\mu^{1}, \ldots, \mu^{m}\right\rangle \in T$ and $j=1, \ldots, m$. Any subset of a full $\mathcal{F}$-transformation tree is called an $\mathcal{F}$ transformation tree. Letting $i d$ denote the identity map, by convention $T:=\{i d\}$ is the unique full $\mathcal{F}$-transformation tree of height 0 .

Note that a full $\mathcal{F}$-transformation tree of height 1 is simply the family of an $\mathcal{F}$ admissible transformation with some choice of domains. Therefore combining the discussion prior to Definition 6.4 with a straightforward induction on $\operatorname{ht}(T)$ shows the following.

Lemma 6.11. For any full $\mathcal{F}$-transformation tree $T$, there is a finite $S \subseteq T$ such that $U=\bigcup_{\mu \in S} \mu\left(B_{\epsilon(\mu)}\right)$ is a neighborhood of the origin. 
The choice of the set $S$ of course depends on the choices of the domains of each of the maps in $T$. Also, by first shrinking the domains of each of the maps in $T, U$ can be made as small as possible.

Given $f \in \mathcal{F}_{n+1, r}$, not only do we want to construct a full $\mathcal{F}$-transformation tree $T$ in $(x, y)$ such that $f \circ \mu(x, y)$ is normal crossings for all $\mu \in T$, but we also want to be able the unwind the coordinate transformations of $\mu$ to prepare $f(x, y)$ back in the original coordinates. Therefore because of Lemmas 6.6 and 6.7. we want $T$ to have the following property.

Definition 6.12. Given a set $T$ of $\mathcal{F}$-transformation sequences in $(x, y), T$ respects $y$ if for every $\mu=\left\langle\mu^{1}, \ldots, \mu^{m}\right\rangle \in T, j \in\{1, \ldots, m-1\}$ and $i \in\{1, \ldots, n\}$, $\mu^{j}$ is not the transformation $b_{\infty}^{i, n+1}$.

Definition 6.13. Given $f_{1}, \ldots, f_{m} \in \mathcal{F}_{n, r}$, the set of functions $\left\{f_{1}, \ldots, f_{m}\right\}$ is normal crossings if each $f_{i}$ is normal crossings, say $f_{i}(x)=x^{\alpha_{i}} u_{i}(x)$ with $\alpha_{i} \in \mathbb{N}^{n}$ and $u_{i}(x)$ a unit, and if $\left\{\alpha_{1}, \ldots, \alpha_{m}\right\}$ is linearly ordered (cf. Definitions 6.1). We use similar terminology for sets of germs in $\mathcal{F}_{n}$.

Lemma 6.14. Let $f_{1}, \ldots, f_{m} \in \mathcal{F}_{n, r}$.

(i) The function $f_{1} \cdots f_{m}$ is normal crossings if and only if $f_{i}$ is normal crossings for all $i=1, \ldots, m$.

(ii) If $f_{i}$ and $f_{i}-f_{j}$ are normal crossings for all $i, j=1, \ldots, m$ such that $f_{i} \neq f_{j}$, then the set of functions $\left\{f_{1}, \ldots, f_{m}\right\}$ is normal crossings.

Proof. See Bierstone and Milman [2, Lemma 4.7].

Theorem 6.15. For every $n \in \mathbb{N}$ and nonzero $f \in \mathcal{F}_{n+1}$, there is a full transformation tree (of germs) $T$ in $(x, y)$ such that for all $\mu=\left\langle\mu^{1}, \ldots, \mu^{m}\right\rangle \in T, f \circ \mu$ is normal crossings; write $f \circ \mu(x, y)=x^{\alpha} y^{d} u(x, y)$ with $\alpha=\left(\alpha_{1}, \ldots, \alpha_{n}\right) \in \mathbb{N}^{n}$, $d \in \mathbb{N}$ and $u(x, y)$ a unit. (We say $T$ "transforms $f$ to normal crossings".) We may choose $T$ so that, in addition, $T$ respects $y$ and

$$
d \geq \alpha_{i}
$$

if $\mu^{m}=b_{\infty}^{i, n+1}$ for some $i \in\{1, \ldots, n\}$.

Corollary 6.16. If $f: \mathbb{R}^{n+1} \rightarrow \mathbb{R}$ is $\mathcal{F}$-analytic on a compact set $A \subseteq \mathbb{R}^{n+1}$, then $f$ is $\mathbb{N}$-prepared on $A$.

As usual, because $\mathcal{F}$ is fixed, we will frequently drop the prefix " $\mathcal{F}-$ " in our terminology.

Before proving the theorem and corollary in general, let us prove them for the simple function $f(x, y)=y^{2}-x$ considered in Example 3.6 in order to get the picture of what is going on. In our construction we shall use only needed blow-up substitutions, so the transformation tree we construct transforming $f$ to normal crossings will be finite. By unwinding the coordinate transformations of this transformation tree, we obtain the preparation of $f$ given in Example 3.6. The cylinder $C_{1}$ is the center of a power substitution, and the cylinders $C_{2}, \ldots, C_{9}$ are obtained from the images of each of the maps in our transformation tree. See Figures 2 and 3 for pictures of the domains of our functions under discussion, showing their zero sets and rough sketches of the preimages of the cylinders $C_{2}, \ldots, C_{9}$. As in Figure 1. our sketches will ignore the overlap of the cylinders.

The idea is to separate the two branches $y=\sqrt{x}$ and $y=-\sqrt{x}$ of the zero set of $f$ by applying blow-up substitutions. But to ensure that our desingularization 


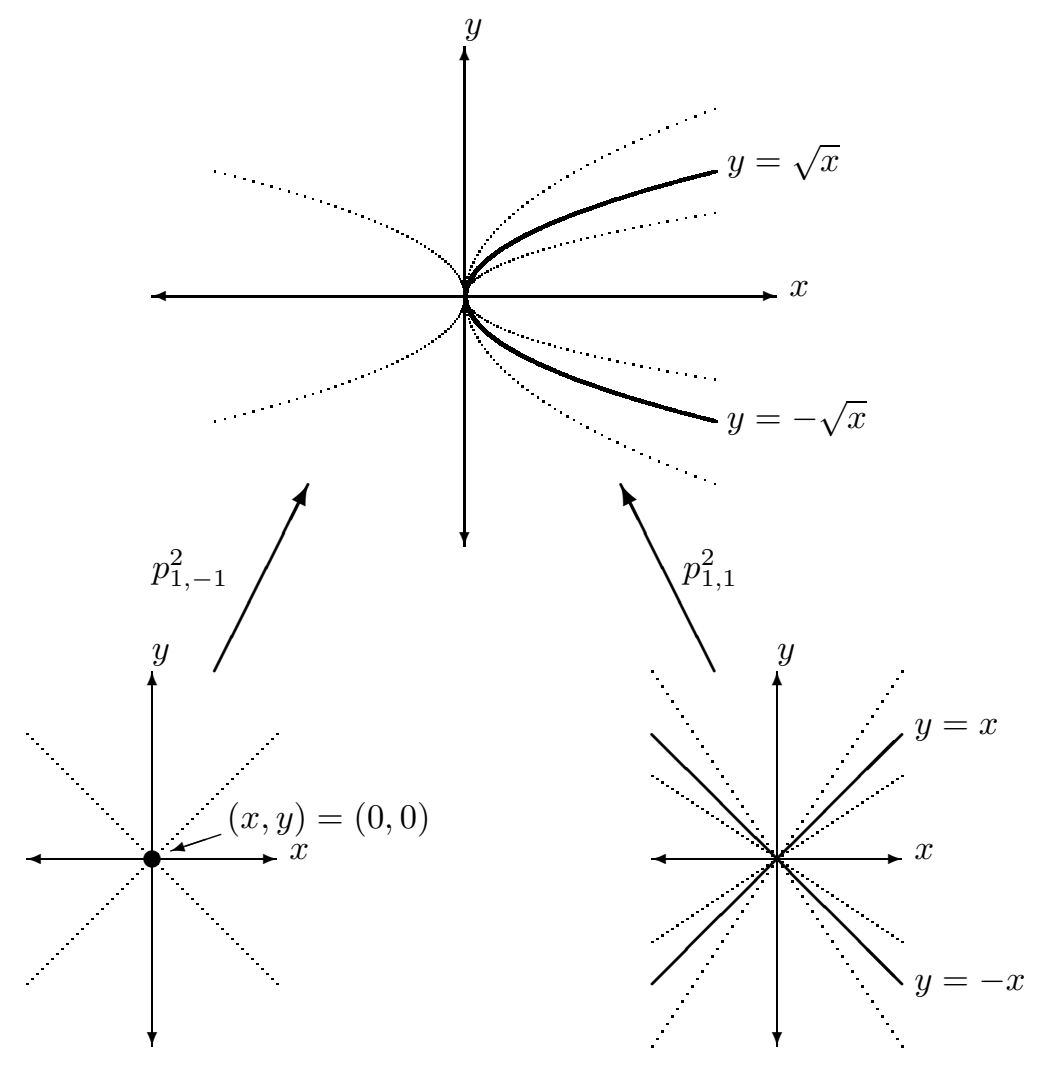

Figure 2. Zero sets of $f(x, y)=y^{2}-x$, and of $f \circ p_{1,1}^{2}$ and $f \circ p_{1,-1}^{2}$.

process stops once a blow-up substitution $b_{\infty}^{1,2}$ is applied, we first want to make the order of our function at 0 (which is 1 ) to be the same as the order in $y$ of our function at 0 (which is 2). This can be done by applying the power substitutions $x \mapsto \pm x^{2}$ to make our new functions of order 2 :

$$
\begin{aligned}
f \circ p_{1,1}^{2}(x, y) & =y^{2}-x^{2}, \\
f \circ p_{1,-1}^{2}(x, y) & =y^{2}+x^{2} .
\end{aligned}
$$

This has the effect of making the branches of the zero sets of the transformed functions analytic at the origin. (In contrast, rather than applying power substitutions in $x$ to increase the total order to the order in $y$, Bierstone and Milman [2] apply a linear coordinate transformation to reduce the order in $y$ to the total order.)

Let $1<\delta<\epsilon$. To transform $f \circ p_{1,1}^{2}$ to normal crossings, apply $b_{\lambda}^{1,2}$ for $\lambda=$ $-1,0,1, \infty$ :

$$
\begin{aligned}
& f \circ p_{1,1}^{2} \circ b_{\infty}^{1,2}(x, y)=y^{2}\left[1-x^{2}\right] \text { for }|x|<\delta^{-1}, \\
& f \circ p_{1,1}^{2} \circ b_{0}^{1,2}(x, y)=x^{2}\left[y^{2}-1\right] \text { for }|y|<\delta^{-1}, \\
& f \circ p_{1,1}^{2} \circ b_{1}^{1,2}(x, y)=x^{2} y[y+2] \text { for } \epsilon^{-1}-1<y<\epsilon-1,
\end{aligned}
$$




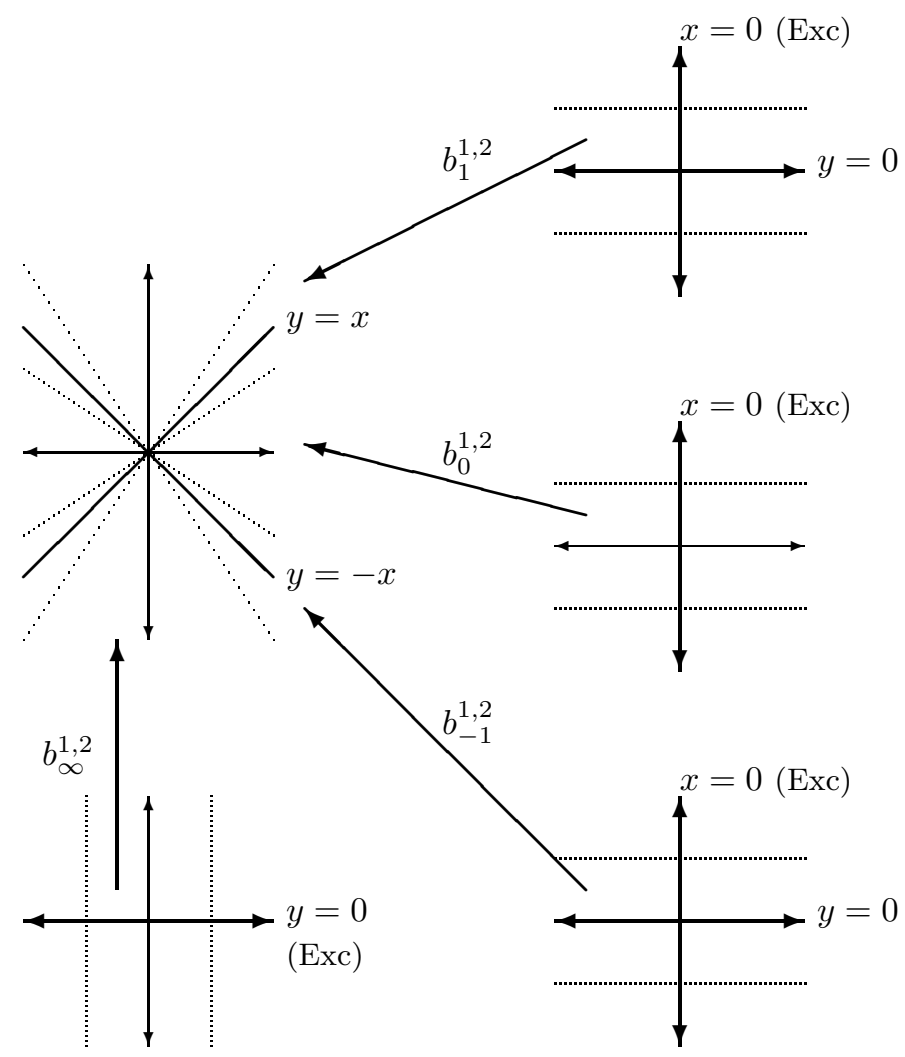

Figure 3. Zero sets of $f \circ p_{1,1}^{2}$, and of $f \circ p_{1,1}^{2} \circ b_{\lambda}^{1,2}$ for $\lambda=\infty,-1,0,1$.

$$
f \circ p_{1,1}^{2} \circ b_{-1}^{1,2}(x, y)=x^{2} y[y-2] \text { for } 1-\epsilon<y<1-\epsilon^{-1},
$$

where expressions in square brackets are units on the specified set.

Consider the picture in the upper left of Figure 3 which shows the zero set of $f \circ p_{1,1}^{2}$. The vertical wedge which is the image of $b_{\infty}^{1,2}$ contains no zero of $f \circ p_{1,1}^{2}$ other than the origin. As a result, the only zero of $f \circ p_{1,1}^{2} \circ b_{\infty}^{1,2}$ is the exceptional divisor of $b_{\infty}^{1,2}, y=0$. This was the point of applying the power substitution; no branch of the zero set of $f \circ p_{1,1}^{2}$ is vertical at the origin, so the process stops once $b_{\infty}^{1,2}$ is applied.

To transform $f \circ p_{1,-1}^{2}$ to normal crossings, apply $b_{\lambda}^{1,2}$ for $\lambda=0, \infty$ :

$$
\begin{array}{ll}
f \circ p_{1,-1}^{2} \circ b_{\infty}^{1,2}(x, y)=y^{2}\left[x^{2}+1\right] & \text { for }|x|<2, \\
f \circ p_{1,-1}^{2} \circ b_{0}^{1,2}(x, y)=x^{2}\left[y^{2}+1\right] & \text { for }|y|<2 .
\end{array}
$$

We omit the picture of the inverse images of the zero set of $f \circ p_{1,-1}^{2}$ under $b_{0}^{1,2}$ and $b_{\infty}^{1,2}$, since it is similar in spirit to Figure 3 .

Thus $f$ has been transformed to normal crossings. By considering the centers of our transformations separately, unwinding the given coordinate transformations gives the $\mathbb{N}$-prepared form of $f$ given in Example 3.6. For example, $f \circ p_{1,1}^{2} \circ$ $b_{1}^{1,2}(x, y)=x^{2} y[y+2]$ for $\epsilon^{-1}-1<y<\epsilon-1$. By applying the "inverse" of $b_{1}^{1,2}$, 
namely $(x, y) \mapsto(x, y / x-1)$ for $x \neq 0$, we get $f \circ p_{1,1}^{2}(x, y)=x^{2}(y / x-1)[(y / x-1)+2]$ for $\epsilon^{-1}-1<y / x-1<\epsilon-1, x \neq 0$. That is, $f \circ p_{1,1}^{2}(x, y)=x(y-x)[(y-x) / x+2]$ for $\epsilon^{-1} x<y<\epsilon x, x \neq 0$. Now applying the "inverse" of $p_{1,1}^{2}$, namely $x \mapsto \sqrt{x}$ for $x>0$, we get that $f(x, y)=\sqrt{x}(y-\sqrt{x})[(y-\sqrt{x}) / \sqrt{x}+2]$ for $\epsilon^{-1} \sqrt{x}<y<\epsilon \sqrt{x}$, $x>0$, which is the prepared form of $f$ on the cylinder $C_{3}$.

Of course, the function $f(x, y)=y^{2}-x$ is quite simple. In general the construction is only local, not global as above, and involves translating by implicitly defined functions.

Proof of Theorem 6.15. By induction on $n$. If $n=0, f(y)$ is normal crossings. So fix $n>0$, and assume the theorem holds for all germs in $\mathcal{F}_{n}$. The following lemma is needed for both the current proof and for later use.

Lemma 6.17. Let $x:=\left(x_{1}, \ldots, x_{n}\right)$ and $z:=\left(z_{1}, \ldots, z_{m}\right)$, and let $f \in \mathcal{F}_{n+m}$. There is a full transformation tree (of germs) $T$ in $x$ such that for each $\mu \in T$, $f \circ \mu(x, z)=x^{\alpha} g(x, z)$ for some $\alpha \in \mathbb{N}^{n}$ and $g \in \mathcal{F}_{n+m}$ such that $g(0, z) \neq 0$.

Proof. Let ${ }^{\wedge}: \mathcal{F}_{n} \rightarrow \mathbb{R} \llbracket x \rrbracket$ denote the map taking $f$ to its Taylor series $\widehat{f}(x)$ at the origin. Write $\widehat{f}(x, z)=\sum_{\beta \in \mathbb{N}^{m}} \widehat{f}_{\beta}(x) z^{\beta}$, and note that $f_{\beta} \in \mathcal{F}_{n}$ for each $\beta$ since $\mathcal{F}$ is closed under differentiation and composition. From the Noetherianity of $\mathbb{R}[[x, z]$, there is a finite $B \subseteq \mathbb{N}^{m}$ such that

$$
\widehat{f}(x, z)=\sum_{\beta \in B} \widehat{f}_{\beta}(x) z^{\beta} u_{\beta}(x, z),
$$

where for each $\beta \in B, \widehat{f}_{\beta}(x) \neq 0$ and $u_{\beta}(x, z) \in \mathbb{R}[[x, z]$ is a formal unit . Let $F(x)$ be the product of all the $f_{\beta}(x)$ and nonzero $f_{\beta}(x)-f_{\gamma}(x)$ for $\beta, \gamma \in B$. By the induction hypothesis in the proof of Theorem 6.15, there is a full transformation tree $T$ in $x$ transforming $F(x)$ to normal crossings. Fix $\mu \in T$. By Lemma 6.14 the set of germs $\left\{f_{\beta} \circ \mu: \beta \in B\right\}$ is normal crossings. So for each $\beta \in B, f_{\beta} \circ \mu(x)=x^{\alpha_{\beta}} v_{\beta}(x)$ for some $\alpha_{\beta} \in \mathbb{N}^{n}$ and unit $v_{\beta}(x)$, and there exists $\gamma \in B$ such that $\alpha:=\alpha_{\gamma} \leq \alpha_{\beta}$ for all $\beta \in B$. Let

$$
G(x, z):=\sum_{\beta \in B} x^{\alpha_{\beta}-\alpha} z^{\beta} \widehat{v}_{\beta}(x) u_{\beta}(x, z),
$$

and observe that $\widehat{f \circ \mu}(x, z)=x^{\alpha} G(x, z)$ and $G(0, z) \neq 0$. Since $f \circ \mu \in \mathcal{F}_{n+m}$ and $\mathcal{F}$ is closed under division by variables, $f \circ \mu(x, z)=x^{\alpha} g(x, z)$ for some $g \in \mathcal{F}_{n+m}$. But $\widehat{g}(x, z)=G(x, z)$, so $g(0, z) \neq 0$ as desired.

To prove Theorem 6.15, apply Lemma 6.17 to $f(x, y)$ to get a full transformation tree $S$ in $x$ such that for each $\mu \in S, f \circ \mu(x, y)=x^{\alpha} g(x, y)$ for some $\alpha \in \mathbb{N}^{n}$ and $g \in \mathcal{F}_{n+1}$ such that $g(0, y) \neq 0$. Now proceed as in the proof of [21, Theorem 2.5] to construct a tree $S_{\mu}$ transforming $f \circ \mu$ to normal crossings. Then $T:=\{\mu \circ \nu$ : $\left.\mu \in S, \nu \in S_{\mu}\right\}$ transforms $f$ to normal crossings. We review the construction of $S_{\mu}$ to observe that $T$ has all the desired properties.

Since $g(0, y) \neq 0, g(x, y)$ has a finite order in the variable $y$. That is, there is a $d \in \mathbb{N}$ such that $\frac{\partial^{d} g}{\partial y^{d}}(0) \neq 0$ and $\frac{\partial^{i} g}{\partial y^{i}}(0)=0$ for $i=0, \ldots, d-1$. In addition to $n$ we also induct on $d$. The case $d=0$ is trivial, since then $g$ is a unit. So we assume that $d>0$. 
If we let $\theta \in \mathcal{F}_{n}$ be the germ implicitly defined by the conditions $\theta(0)=0$ and $\frac{\partial^{d-1} g}{\partial y^{d-1}}(x, \theta(x))=0$, then by taking $\nu$ to be $t_{\theta}$, we may write

$$
g \circ \nu(x, y)=\sum_{i \in I} g_{i}(x) y^{i}+y^{d} u(x, y),
$$

where $u \in \mathcal{F}_{n+1}$ is a unit and $I \subseteq\{0, \ldots, d-2\}$ is such that $g_{i}(x) \neq 0$ for all $i \in I$. Note that $g_{i} \in \mathcal{F}_{n}$ and $g_{i}(0)=0$ for all $i \in I$. We are done if $I$ is empty, so assume otherwise.

Let $G(x)$ be the product of $x^{\alpha}$ and all the $g_{i}$ and nonzero $g_{j}^{d ! /(d-j)}-g_{i}^{d ! /(d-i)}$ for $i, j \in I$. By the induction hypothesis in $n$, there is a full transformation tree in $x$ transforming $G$ to normal crossings. By extending $\nu$ by each of the transformation sequences from this tree, we may assume that the set of germs $\left\{g_{i}^{d ! /(d-i)}: i \in I\right\}$ is normal crossings. By further extending $\nu$ by performing power substitutions in $x$, we may in fact assume that $\left\{g_{i}^{1 /(d-i)}: i \in I\right\}$ is a collection of $\mathcal{F}$-analytic germs which is normal crossings.

So we may write $f \circ \mu(x, y)=x^{\alpha} g \circ \nu(x, y)$ and

$$
g \circ \nu(x, y)=\sum_{i \in I} x^{\beta_{i}} y^{i} v_{i}(x)+y^{d} u(x, y)
$$

with $\left\{\beta_{i} /(d-i): i \in I\right\} \subseteq \mathbb{N}^{n} \backslash\{0\}$ linearly ordered and each $v_{i}(0) \neq 0$. Write $\alpha=\left(\alpha_{1}, \ldots, \alpha_{n}\right)$ and $\beta_{i}=\left(\beta_{i, 1}, \ldots, \beta_{i, n}\right)$, and put $\left|\beta_{i}\right|=\sum_{j=1}^{n} \beta_{i, j}$. In addition to $n$ and $d$, we now also induct on $\sum_{i \in I}\left|\beta_{i}\right|$. Fix $k \in I$ smallest such that $\beta_{k} /(d-k) \leq$ $\beta_{i} /(d-i)$ for all $i \in I$, and fix $1 \leq j \leq n$ such that $\beta_{k, j}>0$. We shall extend $\nu$ by the family of blow-up substitutions $\left\{b_{\lambda}^{j, n+1}: \lambda \in \mathbb{R} \cup\{\infty\}\right\}$.

A simple calculation shows that $g \circ \nu \circ b_{\infty}^{j, n+1}(x, y)=y^{d} U(x, y)$ for some unit $U \in \mathcal{F}_{n+1}$. So

$$
f \circ \nu \circ b_{\infty}^{j, n+1}(x, y)=x^{\alpha} y^{d+\alpha_{j}} U(x, y),
$$

which is normal crossings. Another simple calculation shows that for $\lambda \in \mathbb{R}, g \circ \nu \circ$ $b_{\lambda}^{j, n+1}=x_{j}^{d} G_{\lambda}(x, y)$ for some $G_{\lambda} \in \mathcal{F}_{n+1}$. Since $g \circ \nu(x, y)$ has no term involving $y^{d-1}$, for $\lambda \neq 0$ the order of $G_{\lambda}(x, y)$ in $y$ is less than $d$. On the other hand, either the order of $G_{0}(x, y)$ in $y$ is less than $d$, or $G_{0}(x, y)$ is of the same form as $g \circ \nu(x, y)$ given in (6.5), but the new value of $\sum_{i \in I}\left|\beta_{i}\right|$ is decreased. By our induction hypotheses, this finishes the construction of $T$. We need only observe that $T$ has the desired properties.

First, $T$ was constructed by successively extending transformation sequences by transformation families, and each such extension either transformed $f$ to a germ which is normal crossings in the new coordinates or decreased some parameter $n$, $d$ or $\sum_{i \in I}\left|\beta_{i}\right|$, which can occur only finitely many times. So $T$ has finite height, branches according to transformation families and does in fact transform $f$ to normal crossings. Second, the construction stops once a blow-up substitution $b_{\infty}^{j, n+1}$ is applied, so $T$ respects $y$. Finally, (6.6) verifies (6.3).

Proof of Corollary 6.16, By induction on $n$. By considering each function $f(x+a)$ for $a \in A$ and using the compactness of $A$, it suffices to assume that $f \in \mathcal{F}_{n+1, r}$ for some $r$ and show that $f$ is $\mathbb{N}$-prepared on some neighborhood of the origin. By Theorem 6.15 and Lemma 6.11, there is a finite transformation tree $T$ in $(x, y)$ which respects $y$, is such that $U=\bigcup_{\mu \in T} \mu\left(B_{\epsilon(\mu)}\right)$ is a neighborhood of the origin contained in $B_{r}$, and is such that for each $\mu \in T, f \circ \mu$ is normal crossings and 
(6.3) holds when $\mu$ ends in a blow-up substitution $b_{\infty}^{i, n+1}$. We claim that $f$ is $\mathbb{N}$ prepared on $U$. Indeed, put $T_{1}=\left\{\mu^{1}:\left\langle\mu^{1}, \ldots, \mu^{m}\right\rangle \in T\right\}$ and $T_{\mu}=\left\{\left\langle\nu^{2}, \ldots, \nu^{m}\right\rangle\right.$ : $\left.\left\langle\mu, \nu^{2}, \ldots, \nu^{m}\right\rangle \in T\right\}$ for each $\mu \in T_{1}$. By inducting on $\operatorname{ht}(T)$ we may assume that $f \circ \mu$ is $\mathbb{N}$-prepared on $U_{\mu}=\bigcup_{\nu \in T_{\mu}} \nu\left(B_{\epsilon(\mu \circ \nu)}\right)$ for each $\mu \in T_{1}$. Then by applying Lemmas 6.6 and 6.7 we get that $f$ is $\mathbb{N}$-prepared on $U \backslash \operatorname{Cen}\left(T_{1}\right)$. But $\operatorname{Cen}\left(T_{1}\right)$ is the (possibly empty) intersection of coordinate hyperplanes of codimension 1. The function $f(x, 0)$ is simply a term in $x$, and by the induction hypothesis in $n$, $\left.f(x, y)\right|_{x_{i}=0}$ is $\mathbb{N}$-prepared on $B_{r}$ for $i=1, \ldots, n$. So $f$ is $\mathbb{N}$-prepared on $B_{r} \cap$ $\operatorname{Cen}\left(T_{1}\right)$, and hence on $U$.

Remark 6.18. The full strength of our assumption that $\mathcal{F}$ is a Weierstrass system was not used in the proof of Theorem 6.15. The assumption of closure under Weierstrass preparation may be weakened to the assumptions of closure under division by variables and closure under implicit functions (cf. Remarks 2.3). This weaker set of assumptions is advantageous because in any expansion of the real field, the collection of definable functions is clearly closed under the operations of composition, division by variables and implicit definition of functions. I do not know whether Weierstrass preparation preserves definability in general, but this seems unlikely (cf. Section 9).

Furthermore, if one wishes to work with a collection of real valued $C^{\infty}$ functions with real domains, the proof of Theorem 6.15 also shows that the assumption of analyticity can be weakened to the assumption of quasianalyticity, that is, that the Taylor map ${ }^{\wedge} \mathcal{F}_{n} \rightarrow \mathbb{R} \llbracket x \rrbracket$ is injective. This is of interest because of the following two examples of such systems of functions:

1. the quasianalytic Denjoy-Carleman classes considered, for instance, in [3], 4] and [21;

2. given any polynomially bounded o-minimal expansion of the real field, the collection of functions $f: B_{r} \rightarrow \mathbb{R}$ which expand to a definable $C^{\infty}$ function $f: U \rightarrow \mathbb{R}$ on some neighborhood $U$ of $B_{r}$ (see C. Miller [18]); the collection of $C^{\infty}$ sub-Pfaffian functions is a particular example.

Because of the heavy reliance in the proof of the preparation theorem on Theorem 6.15, it is tempting to ask whether $\mathbb{R}_{\mathcal{F}}^{\mathbb{Q}}$ has a preparation theorem when $\mathcal{F}$ is such a quasianalytic class of functions subject to this weaker set of assumptions. As mentioned in the Introduction, this question (in full strength) remains open. We shall see that the key step in our proof, found in the proof of Lemma 7.5. uses Weierstrass preparation in a seemingly essential way (cf. Remark 7.6). In a possibly less essential way, our proof will also use the assumption of analyticity in its reliance on Lemma 7.2 in the proof of Proposition 7.7.

\section{Proof of the Main Proposition}

This section completes the proof of the Main Theorem in the case of $K=\mathbb{Q}$ by proving Proposition 5.1.

Definition 7.1. For any sets $A \subseteq D \subseteq \mathbb{C}^{n}$ and function $f: D \rightarrow \mathbb{C}$ let

$$
Z_{A}(f):=\{x \in A: f(x)=0\} .
$$

Lemma 7.2. Consider $f, g, h \in \mathcal{F}_{n, r}$ such that $g$ and $h$ are normal crossings, and put $B=\mathcal{B}_{r}$ and $E=Z_{B}(h)$. If $Z_{B \backslash E}(f) \subseteq Z_{B \backslash E}(g)$, then $f$ is normal crossings. 
Proof. Since $g$ and $h$ are normal crossings, so is their product. Put $g(x) h(x)=$ $x^{\alpha} u(x)$ with $\alpha \in \mathbb{N}^{n}$ and $u$ a unit on $B$. Since $Z_{B \backslash E}(f) \subseteq Z_{B \backslash E}(g), Z_{B}(f) \subseteq$ $Z_{B}(g) \cup Z_{B}(h)=Z_{B}(g h)=Z_{B}\left(x^{\alpha}\right)$. Fix $\beta \leq \alpha$ minimal such that $Z_{B}(f) \subseteq Z_{B}\left(x^{\beta}\right)$ (so $\beta \in\{0,1\}^{n}$ ).

We claim that $Z_{B}(f)=Z_{B}\left(x^{\beta}\right)$. To see this, apply the fact that the set of regular zeros of a holomorphic function is dense in its set of zeros, and hence there is a set $U \subseteq B$ which is open and dense in $B$ and is such that $Z_{U}(f)$ and $Z_{U}\left(x^{\beta}\right)$ are both $(n-1)$-dimensional complex manifolds. But $Z_{U}(f) \subseteq Z_{U}\left(x^{\beta}\right)$, so in fact $Z_{U}(f)=Z_{U}\left(x^{\beta}\right)$ by dimensional considerations. Since the closed sets $Z_{B}(f)$ and $Z_{B}\left(x^{\beta}\right)$ agree on a dense subset, they must be equal.

Now apply the claim to $f$ to get $f_{1} \in \mathcal{F}_{n, r}$ such that $f(x)=x^{\beta} f_{1}(x)$. But $f_{1}$ satisfies the same hypotheses as $f_{1}$, so we are done if $f_{1}$ is a unit and can apply the claim to $f_{1}$ otherwise. By repeatedly applying the claim as such, a process which must eventually stop since $f$ has finite order, we prove the lemma.

Our goal is to $\mathbb{Z}$-prepare a function of the form $f\left(x_{1}, \ldots, x_{n-1}, x_{n} / y, y\right)$ for an $\mathcal{F}$-analytic function $f$. Our strategy in doing this is modeled after Parusiński's proof of his preparation theorem [19, Theorem 7.5]. We consider the slightly more general form $F(x, y)=f(x, g(x) / y, y)$ for $\mathcal{F}$-analytic functions $f$ and $g$ and construct a finite transformation tree $T$ respecting $y$ such that $F \circ \mu(x, y)$ is normal crossings for each $\mu \in T$ and such that $\left\{(x, y) \in \mathbb{R}^{n+1}: y \neq 0,|x|,|g(x) / y|,|y|<\right.$ $\delta\} \subseteq \bigcup_{\mu \in T} \mu\left(B_{\epsilon(\mu)}\right)$ for some $\delta>0$. Since $T$ respects $y$, we can unwind its transformations to prepare $F$ back in the original coordinates. To construct $T$ we will first use Weierstrass preparation to construct a full transformation tree $S$ in $x$ such that for each $\mu \in S$ there is an $\mathcal{F}$-analytic function $\Phi_{\mu}(x, y)$ such that the complex zero set of $F \circ \mu$ is contained in the complex zero set of $\Phi_{\mu}$. We then apply Theorem 6.15 to construct a full transformation tree $S_{\mu}$ in $(x, y)$ which respects $y$ and is such that for all $\nu \in S_{\mu}$, either $\Phi_{\mu} \circ \nu$ is normal crossings and $F \circ \mu \circ \nu$ is analytic, or else the image of $\mu \circ \nu$ misses the set $\left\{(x, y) \in \mathbb{R}^{n+1}: y \neq 0,|x|,|g(x) / y|,|y|<\delta\right\}$ for sufficiently small $\delta>0$. In the first case Lemma 7.2 shows that $F \circ \mu \circ \nu$ must also be normal crossings, and in the second case $\mu \circ \nu$ is irrelevant and may be ignored. Thus $T$ can be extracted as a suitable finite subset of the full transformation tree $\left\{\mu \circ \nu: \mu \in S, \nu \in S_{\mu}\right\}$.

Just as in the proof of Theorem 6.15 the construction of $\left\{\mu \circ \nu: \mu \in S, \nu \in S_{\mu}\right\}$ is completely local. So instead of cluttering up the construction by keeping track of continually shrinking neighborhoods, it is more convenient to phrase most of the argument in terms of germs, for which the following notation will be useful.

Definition 7.3. Consider a function $\varphi: D \rightarrow \mathbb{C}^{m}$ defined on a set $D \subseteq \mathbb{C}^{n}$ such that $0 \in \operatorname{cl}(D)$ and also a function $f: U \rightarrow \mathbb{C}$ defined on a neighborhood $U \subseteq \mathbb{C}^{m}$ of the origin. For any $V \subseteq U$ and $V^{\prime} \subseteq \mathbb{C}^{n}$ let

$$
Z_{V^{\prime}}\left(\left.f\right|_{V} \circ \varphi\right)=\left\{x \in D \cap V^{\prime}: \varphi(x) \in V, f \circ \varphi(x)=0\right\} .
$$

Given a function $g: W \rightarrow \mathbb{C}$ defined on a neighborhood $W \subseteq \mathbb{C}^{n}$ of the origin, we write

$$
Z(f \mid \circ \varphi) \subseteq Z(g)
$$

to mean that $Z_{V^{\prime}}\left(\left.f\right|_{V} \circ \varphi\right) \subseteq Z_{V^{\prime}}(g)$ for some neighborhoods $V \subseteq U$ and $V^{\prime} \subseteq W$ of the origins in their respective spaces. 
In other words, $Z(f \mid \circ \varphi) \subseteq Z(g)$ simply means that for all $x \in \mathbb{C}^{n}, f \circ \varphi(x)=0$ implies that $g(x)=0$ provided that $\varphi(x)$ is defined and both $x$ and $\varphi(x)$ are sufficiently small. This concept can therefore also be applied to the germs of $f, \varphi$ and $g$ at the origins in their respective spaces. Similarly, given any germ at 0 of a continuous function $\mu: \mathbb{C}^{n} \rightarrow \mathbb{C}^{n}$ such that $\mu(0)=0$, this concept can be applied to the germs of $f, \varphi \circ \mu$ and $g \circ \mu$. We shall frequently use the obvious fact that given such a germ $\mu$, if $Z(f \mid \circ \varphi) \subseteq Z(g)$, then $Z(f \mid \circ \varphi \circ \mu) \subseteq Z(g \circ \mu)$.

Lemma 7.4. Let $\lambda \in \mathbb{R}, f \in \mathcal{F}_{n+1}$ and $g \in \mathcal{F}_{n}$ be such that $g(0)=0$. Let $D$ be the germ at 0 of $\left\{(x, y) \in \mathbb{C}^{n+1}: y \neq 0\right\}$, and define the germ $\varphi: D \rightarrow \mathbb{C}^{n+1}$ by $\varphi(x, y)=(x, g(x) / y-\lambda y)$. Then there is a full $\mathcal{F}$-transformation tree (of germs) $T$ in $x$ such that for each $\mu \in T$ there exists $\Phi_{\mu} \in \mathcal{F}_{n+1}$ such that $Z(f \mid \circ \varphi \circ \mu) \subseteq Z\left(\Phi_{\mu}\right)$.

Proof. By Lemma 6.17 there is a full transformation tree $T$ in $x$ such that for all $\mu \in T, f \circ \mu(x, y)=x^{\alpha} h(x, y)$ for some $\alpha \in \mathbb{N}^{n}$ and $h \in \mathcal{F}_{n+1}$ such that $h(0, y) \neq 0$. Let $d$ be the order of $h(0, y)$. Fix $\mu \in T$ and the corresponding $\alpha, h$ and $d$. Put $\mu(x, y)=\left(\mu^{\prime}(x), y\right)$, where $\mu^{\prime}$ is an admissible transformation in $x$.

By Weierstrass preparation $h(x, y)=w(x, y) u(x, y)$ for some $w, u \in \mathcal{F}_{n+1}$, where $w$ is a Weierstrass polynomial in $y$ of degree $d$ and $u$ is a unit. So $f \circ \varphi \circ \mu(x, y)=$ $f\left(\mu^{\prime}(x), g \circ \mu^{\prime}(x) / y-\lambda y\right)$ is the product of $x^{\alpha} w\left(x, g \circ \mu^{\prime}(x) / y-\lambda y\right)$ and a unit. By letting $\Phi_{\mu}(x, y)=x^{\alpha} y^{d} w\left(x, g \circ \mu^{\prime}(x) / y-\lambda y\right)$, which is a polynomial in $y$ having coefficients in $\mathcal{F}_{n}$, we see that $Z(f \mid \circ \varphi \circ \mu) \subseteq Z\left(\Phi_{\mu}\right)$.

Lemma 7.5. Let $f \in \mathcal{F}_{n+2}$ and $g \in \mathcal{F}_{n}$ be such that $g(0)=0$. Let $D$ be the germ at 0 of $\left\{(x, y) \in \mathbb{C}^{n+1}: y \neq 0\right\}$, and define the germ $\varphi: D \rightarrow \mathbb{C}^{n+2}$ by $\varphi(x, y)=(x, g(x) / y, y)$. Then there is a full $\mathcal{F}$-transformation tree (of germs) $T$ in $x$ such that for each $\mu \in T$ there exists $\Phi_{\mu} \in \mathcal{F}_{n+1}$ such that $Z(f \mid \circ \varphi \circ \mu) \subseteq Z\left(\Phi_{\mu}\right)$.

Proof. Let $z$ denote a single variable. By Lemma 6.17 there is a full transformation tree $S$ in $x$ such that for each $\nu \in S, f \circ \nu(x, y, z)=x^{\alpha} h(x, y, z)$ for some $\alpha \in \mathbb{N}^{n}$ and $h \in \mathcal{F}_{n+2}$ such that $h(0, y, z) \neq 0$. Fix $\nu \in S$ and the corresponding $\alpha$ and $h$. Since $\nu$ is in $x$, we may consider it to be a map in $(x, y)$ and put $\nu(x, y)=\left(\nu^{\prime}(x), y\right)$ for some admissible transformation $\nu^{\prime}$ in $x$. So

$$
f \circ \varphi \circ \nu(x, y)=f\left(\nu^{\prime}(x), g \circ \nu^{\prime}(x) / y, y\right)=x^{\alpha} h\left(x, g \circ \nu^{\prime}(x) / y, y\right) .
$$

We now use a method of Parusiński's to study the complex zero set of $f \circ \varphi \circ \nu$. Fix $\lambda \in \mathbb{R}$ such that $h(x, y+\lambda z, z)$ is regular in $z$, which may be done since $h(0, y, z) \neq 0$. By Weierstrass preparation,

$$
h(x, y+\lambda z, z)=w(x, y, z) u(x, y, z)
$$

for some $w, u \in \mathcal{F}_{n+2}$, where $w$ is a Weierstrass polynomial in $z$ and $u$ is a unit. Now consider $(a, b) \in \mathbb{C}^{n+1}$ such that $b \neq 0$, put $c=g \circ \nu^{\prime}(a) / b-\lambda b$, and assume that $(a, b)$ and $\varphi \circ \nu(a, b)$ are sufficiently small so that (7.1) and (7.2) make sense as functions, not just germs, evaluated at $(x, y)=(a, b)$ and $(x, y, z)=(a, c, b)$, respectively. Suppose that $h\left(a, g \circ \nu^{\prime}(a) / b, b\right)=0$. Then $w(a, c, b)=0$ and $\lambda b^{2}+c b-g \circ \nu^{\prime}(a)=0$, so the two polynomials in $y, w(a, c, y)$ and $\lambda y^{2}+c y-g \circ \nu^{\prime}(a)$, have a common root $b$, and hence a common factor. Letting $R(x, z)$ be the resultant in $y$ of $w(x, z, y)$ and $\lambda y^{2}+z y-g \circ \nu^{\prime}(x)$, it follows that $R(a, c)=0$. Observe that $R \in \mathcal{F}_{n+1}$, and put $\psi_{\nu}(x, y)=\left(x, g \circ \nu^{\prime}(x) / y-\lambda y\right)$ and $F_{\nu}(x, y)=x^{\alpha} R(x, y)$. This argument and (7.1) show that $Z(f \mid \circ \varphi \circ \nu) \subseteq Z\left(F_{\nu} \mid \circ \psi_{\nu}\right)$. 
Now apply Lemma 7.4 to obtain a full transformation tree $S_{\nu}$ in $x$ such that for each $\eta \in S_{\nu}, Z\left(F_{\nu} \mid \circ \psi_{\nu} \circ \eta\right) \subseteq Z\left(\Phi_{\nu \circ \eta}\right)$ for some $\Phi_{\nu \circ \eta} \in \mathcal{F}_{n+1}$. But

$$
Z(f \mid \circ \varphi \circ \nu \circ \eta) \subseteq Z\left(F_{\nu} \mid \circ \psi_{\nu} \circ \eta\right)
$$

so if we put $T=\left\{\nu \circ \eta: \nu \in S, \eta \in S_{\mu}\right\}$ we get that $Z(f \mid \circ \varphi \circ \mu) \subseteq Z\left(\Phi_{\mu}\right)$ for all $\mu=\nu \circ \eta \in T$.

Remark 7.6. So as not to lose the main point among all the details, it should be pointed out that Lemma 7.5 is the key step in the proof of the preparation theorem presented here. It is essentially a quantifier elimination result and makes full use of our assumption of closure under Weierstrass preparation. The proof uses Weierstrass preparation and polynomial algebra to show that the image of $\left\{(x, y, t): f\left(\nu^{\prime}(x), t+\lambda y, y\right)=0, \lambda y^{2}+t y-g \circ \nu^{\prime}(x)=0\right\}$ under the projection $(x, y, t) \mapsto(x, t)$ is contained in the zero set of an $\mathcal{F}$-analytic function. This seems like a very essential use of Weierstrass preparation. Lemma 7.5 also uses Weierstrass preparation in its reliance on Lemma 7.4, but this is done out of convenience rather than necessity (see Remark 7.10).

Proposition 7.7. Let $f \in \mathcal{F}_{n+2, s}$ and let $g \in \mathcal{F}_{n, r}$ be such that $g(0)=0$. Let $D=\left\{(x, y) \in \mathcal{B}_{r} \times \mathbb{C}: y \neq 0\right\}$, and define $\varphi: D \rightarrow \mathbb{C}^{n+2}$ by $\varphi(x, y)=(x, g(x) / y, y)$. Then there is a finite $\mathcal{F}$-transformation tree $T$ in $(x, y)$ which respects $y$ as described below. For $\mu \in T$ we write $\mu=\left(\mu_{1}, \ldots, \mu_{n+1}\right)$ and $\mu^{\prime}=\left(\mu_{1}, \ldots, \mu_{n}\right)$; so with this notation

$$
\varphi \circ \mu(x, y)=\left(\mu^{\prime}(x, y), g \circ \mu^{\prime}(x, y) / \mu_{n+1}(x, y), \mu_{n+1}(x, y)\right) .
$$

For all $\mu \in T$ the following hold:

(i) $g \circ \mu^{\prime}$ and $\mu_{n+1}$ are both normal crossings on $\mathcal{B}_{\epsilon(\mu)}$; put $g \circ \mu^{\prime}(x, y)=$ $(x y)^{\alpha(\mu)} u(x, y)$ and $\mu_{n+1}(x, y)=(x y)^{\beta(\mu)} v(x, y)$ for $\alpha(\mu), \beta(\mu) \in \mathbb{N}^{n+1}$ and units $u, v \in \mathcal{F}_{n+1, \epsilon(\mu)}$;

(ii) $\alpha(\mu) \geq \beta(\mu)$; it follows that there is an analytic function $\Phi_{\mu} \in\left(\mathcal{F}_{n+1, \epsilon(\mu)}\right)^{n+2}$ such that $\varphi \circ \mu=\Phi_{\mu}$ on $\left\{(x, y) \in \mathcal{B}_{\epsilon(\mu)}:(x y)^{\beta(\mu)} \neq 0\right\}$;

(iii) $f \circ \Phi_{\mu}$ is normal crossings on $\mathcal{B}_{\epsilon(\mu)}$;

(iv) there are a neighborhood $U \subseteq \mathbb{R}^{n+1}$ of the origin and $\delta>0$ such that

$$
\{(x, y) \in U:|g(x)| \leq \delta|y|\} \subseteq \bigcup_{\mu \in T} \mu\left(B_{\epsilon(\mu)}\right) .
$$

Proof. By Lemma 7.5 there is a full transformation tree (of germs) $S_{1}$ in $x$ such that for all $\nu \in S_{1}, Z(f \mid \circ \varphi \circ \nu) \subseteq Z\left(\Psi_{\nu}\right)$ for some $\Psi_{\nu} \in \mathcal{F}_{n+1}$. Put

$$
\Upsilon_{\nu}(x, y)=\Psi_{\nu}(x, y) \cdot g \circ \nu^{\prime}(x) \cdot y \cdot\left(y-g \circ \nu^{\prime}(x)\right),
$$

where $\nu(x, y)=\left(\nu^{\prime}(x), y\right)$. By Theorem 6.15 there is a full transformation tree (of germs) $S_{1, \nu}$ in $(x, y)$ which respects $y$ and transforms $\Upsilon_{\nu}$ to normal crossings. Note that

$$
Z(f \mid \circ \varphi \circ \nu \circ \eta) \subseteq Z\left(\Psi_{\nu} \circ \eta\right)
$$

for each $\eta \in S_{1, \nu}$. Put $S_{2}=\left\{\nu \circ \eta: \nu \in S_{1}, \eta \in S_{1, \nu}\right\}$. Since $S_{1}$ is in $x$ and each $S_{1, \nu}$ respects $y, S_{2}$ also respects $y$. We now choose functions on suitable domains which are representatives of each of the germs in $S_{2}$.

For each $\mu \in S_{2}$ choose $\epsilon(\mu) \in(0, \infty)^{n+1}$ so that there is an admissible transformation in $\left(\mathcal{F}_{n+1, \epsilon(\mu)}\right)^{n+1}$ whose germ at 0 is $\mu$. We also call this function $\mu$ and now 
consider $S_{2}$ to be a transformation tree of functions, not of germs. Consider some fixed $\mu \in S_{2}$, and put $\mu=\nu \circ \eta$ for $\nu \in S_{1}$ and $\eta \in S_{1, \nu}$. By possibly shrinking $\epsilon(\mu)$, we can ensure that $\mu^{\prime}\left(\mathcal{B}_{\epsilon(\mu)}\right) \subseteq \mathcal{B}_{r}$ and that $\Upsilon_{\nu} \circ \eta$ is normal crossings on $\mathcal{B}_{\epsilon(\mu)}$. So by Lemma 6.14 the functions $\Psi_{\nu} \circ \eta, g \circ \mu^{\prime}$ and $\mu_{n+1}$ are all normal crossings on $\mathcal{B}_{\epsilon(\mu)}$, and using the notation established in statement (i), either $\alpha(\mu) \geq \beta(\mu)$ or $\alpha(\mu) \leq \beta(\mu)$. Since $\mu$ is currently fixed, we shall simply write $\alpha$ and $\beta$ instead of $\alpha(\mu)$ and $\beta(\mu)$.

It follows from (7.3) that by possibly shrinking $\epsilon(\mu)$, and by choosing $\delta(\mu)>0$ sufficiently small, we may assume that

$$
Z_{D(\epsilon, \delta ; \mu)}(f \circ \varphi \circ \mu) \subseteq Z_{D(\epsilon, \delta ; \mu)}\left(\Psi_{\nu} \circ \eta\right)
$$

where

$$
D(\epsilon, \delta ; \mu)=\left\{(x, y) \in \mathcal{B}_{\epsilon(\mu)}: \mu_{n+1}(x, y) \neq 0,\left|g \circ \mu^{\prime}(x, y) / \mu_{n+1}(x, y)\right|<\delta(\mu)\right\} .
$$

The proof now breaks down into three cases depending on the relationship between $\alpha$ and $\beta$.

First suppose that $\alpha \geq \beta$ and $\alpha \neq \beta$. Then by possibly shrinking $\epsilon(\mu)$ we may assume that $\left|(x y)^{\alpha-\beta} u(x, y) / v(x, y)\right|<\delta(\mu)$ on $\mathcal{B}_{\epsilon(\mu)}$. So $D(\epsilon, \delta ; \mu)=\{(x, y) \in$ $\left.\mathcal{B}_{\epsilon(\mu)}:(x y)^{\beta} \neq 0\right\}$, and by putting

$$
\Phi_{\mu}(x, y)=\left(\mu^{\prime}(x, y),(x y)^{\alpha-\beta} u(x, y) / v(x, y),(x y)^{\beta} v(x, y)\right),
$$

we get that $\Phi_{\mu}$ is is a function in $\left(\mathcal{F}_{n+1, \epsilon(\mu)}\right)^{n+2}$ which agrees with $\varphi \circ \mu$ on $D(\epsilon, \delta ; \mu)$. Hence $Z_{D(\epsilon, \delta ; \mu)}\left(f \circ \Phi_{\mu}\right) \subseteq Z_{D(\epsilon, \delta ; \mu)}\left(\Psi_{\nu} \circ \eta\right)$, so by Lemma 7.2 the function $f \circ \Phi_{\mu}$ is normal crossings on $\mathcal{B}_{\epsilon(\mu)}$.

Next suppose that $\alpha=\beta$. Then by shrinking $\delta(\mu)$ we can assume that $|u / v|>$ $\delta(\mu)$ on $\mathcal{B}_{\epsilon(\mu)}$, so $D(\epsilon, \delta ; \mu)$ is empty.

Finally, suppose that $\alpha \leq \beta$ and $\alpha \neq \beta$. Then by shrinking $\epsilon(\mu)$ we may assume that $\left|(x y)^{\alpha-\beta} u(x, y) / v(x, y)\right|>\delta(\mu)$ for all $(x, y) \in \mathcal{B}_{\epsilon(\mu)}$ such that $\mu_{n+1}(x, y) \neq 0$. Again, $D(\epsilon, \delta ; \mu)$ is empty.

For each $\mu \in S_{2}$ the values of $\epsilon(\mu)$ and $\delta(\mu)$ are now set. We extract the desired finite tree $T$ as a suitable subset of $S_{2}$. By Lemma 6.11 there is a finite $S_{3} \subseteq S_{2}$ such that $U=\bigcup_{\mu \in S_{3}} \mu\left(B_{\epsilon(\mu)}\right)$ is a neighborhood of the origin in $\mathbb{R}^{n+1}$. Let $\delta=\min \left\{\delta(\mu): \mu \in S_{3}\right\}$. It follows that

$$
\{(x, y) \in U: y \neq 0,|g(x) / y|<\delta\} \subseteq \bigcup_{\mu \in S_{3}} \mu(D(\epsilon, \delta ; \mu)) .
$$

Put $T=\left\{\mu \in S_{3}: \alpha(\mu) \geq \beta(\mu), \alpha(\mu) \neq \beta(\mu)\right\}$. Since $D(\epsilon, \delta ; \mu)=\emptyset$ for all $\mu \in S_{3} \backslash T$, (7.4) holds with $T$ in place of $S_{3}$, so $\{(x, y) \in U:|g(x)| \leq \delta|y|\} \subseteq$ $\bigcup_{\mu \in T} \mu\left(B_{\epsilon(\mu)}\right)$.

There are now only two things left to do to prove Proposition 5.1 1) observe that preparing $f(x, g(x) / y, y)$ is easy for values of $(x, y)$ for which $(x, g(x) / y, y)$ is not small; 2) unwind the transformations of $T$ given by the previous proposition to prepare $f(x, g(x) / y, y)$ for values of $(x, y)$ for which $(x, g(x) / y, y)$ is small. For both we need the following obvious fact.

Lemma 7.8. For $\mathcal{L}_{\mathcal{F}}^{\mathbb{Q}}$-terms $s_{1}(x), \ldots, s_{N}(x), t_{1}(x)$ and $t_{2}(x)$, let

$$
L(x, y)=\left(s_{1}(x), \ldots, s_{N}(x), t_{1}(x) y+t_{2}(x)\right),
$$


and let $C \subseteq \mathbb{R}^{N+1}$ be an $\mathcal{L}_{\mathcal{F}}^{\mathbb{Q}}$-cylinder. Then

(i) $L^{-1}(C)$ is a finite union of $\mathcal{L}_{\mathcal{F}}^{\mathbb{Q}}$-cylinders;

(ii) if $f: \mathbb{R}^{N+1} \rightarrow \mathbb{R}$ is $\mathcal{L}_{\mathcal{F}}^{\mathbb{Q}}$-prepared on $C$, then $f \circ L$ is $\mathcal{L}_{\mathcal{F}}^{\mathbb{Q}}$-prepared on $L^{-1}(C)$; the same holds for both $\mathbb{N}$ - and $\mathbb{Z}$-preparation.

Lemma 7.9. Let $f \in \mathcal{F}_{n+2, s}$ and let $g \in \mathcal{F}_{n, r}$ be such that $g(0)=0$. Let $D=$ $\left\{(x, y) \in \mathcal{B}_{r} \times \mathbb{C}: y \neq 0\right\}$, and define $\varphi: D \rightarrow \mathbb{C}^{n+2}$ by $\varphi(x, y)=(x, g(x) / y, y)$. Let $A \subseteq D \cap \mathbb{R}^{n+1}$ be a finite union of $\mathcal{L}_{\mathcal{F}}^{\mathbb{Q}}$-cylinders such that $\varphi(A) \subseteq B_{s}$. If $y \sim \psi(x)$ on $A$ for some $\mathcal{L}_{\mathcal{F}}^{\mathbb{Q}}$-term $\psi(x)$, then $f \circ \varphi$ is $\mathbb{N}$-prepared on $A$.

Proof. Since $y \neq 0$ on $A$ and $y \sim \psi(x)$ on $A, \psi(x) \neq 0$ on $A$. By considering $\{(x, y) \in A: \psi(x)>0\}$ and $\{(x, y) \in A: \psi(x)<0\}$ separately, we may assume that $\psi$ has constant sign on $A$. Both sign conditions are handled similarly, so we assume that $\psi(x)>0$ on $A$.

Fix $0<a<b$ such that $a \psi(x) \leq y \leq b \psi(x)$ on $A$. For each $\lambda \in[a, b]$ let $A_{\lambda}=$ $\left\{(x, y) \in A: \frac{1}{2} \lambda \psi(x)<y<\frac{3}{2} \lambda \psi(x)\right\}$. By choosing $a=\lambda_{1}<\cdots<\lambda_{k}=b$ such that $\lambda_{i+1} / \lambda_{i}<3$ for $i=1, \ldots, k-1$, we have that $A \subseteq \bigcup_{i=1}^{k} A_{\lambda_{i}}$. So by considering each $A_{\lambda_{i}}$ and $\lambda_{i} \psi(x)$ separately, we may simply assume that $\frac{1}{2} \psi(x)<y<\frac{3}{2} \psi(x)$ on $A$. Therefore, since $\varphi(A) \subseteq B_{s}$, the following hold on $A$ :

$$
\begin{aligned}
& \left|\frac{g(x)}{\psi(x)}\right|=\left|\frac{y}{\psi(x)} \cdot \frac{g(x)}{y}\right|<\frac{3}{2} s_{n+1}, \\
& |\psi(x)| \leq 2|y| \leq 2 s_{n+2}, \\
& \left|\frac{y-\psi(x)}{\psi(x)}\right| \leq \frac{1}{2} .
\end{aligned}
$$

View $\left.\varphi\right|_{A}$ as the composition of maps $\varphi=\varphi_{2} \circ \varphi_{1}$, where $\varphi_{1}: A \rightarrow \mathbb{R}^{n+3}$ and $\varphi_{2}: \mathbb{R}^{n+2} \times(-1,1) \rightarrow \mathbb{R}^{n+2}$ are defined by

$$
\begin{aligned}
\varphi_{1}(x, y) & =(g(x) / \psi(x), x, \psi(x),(y-\psi(x)) / \psi(x)), \\
\varphi_{2}(w, x, y, z) & =(x, w /(1+z), y(1+z)) .
\end{aligned}
$$

Put $\delta=\left(3 s_{n+1} / 2, s_{1}, \ldots, s_{n}, 2 s_{n+2}, 1 / 2\right)$, and note that $\varphi_{1}(A) \subseteq B_{\delta}$ and that $\varphi_{2}$ is $\mathcal{F}$-analytic on $B_{\delta}$. Therefore by Corollary 6.16, $f \circ \varphi_{2}$ is $\mathbb{N}$-prepared on $B_{\delta}$. So by Lemma 7.8, $f \circ \varphi_{2} \circ \varphi_{1}$ is $\mathbb{N}$-prepared on $A$.

Proof of Proposition 5.1. It suffices to prove the following statement by induction on $n$ :

Consider a bounded function $\varphi: A \rightarrow \mathbb{R}^{n+2}$ of the form

$$
\varphi(x, y)=(x, g(x) / y, y),
$$

where $A \subseteq\left\{(x, y) \in \mathbb{R}^{n+1}: y \neq 0\right\}$ is a bounded set which is the finite union of $\mathcal{L}_{\mathcal{F}}^{\mathbb{Q}}$-cylinders and $g: \mathbb{R}^{n} \rightarrow \mathbb{R}$ is $\mathcal{F}$-analytic on $\mathrm{cl}\left(\Pi_{n}(A)\right)$. If $f: \mathbb{R}^{n+2} \rightarrow \mathbb{R}$ is $\mathcal{F}$-analytic on $\operatorname{cl}(\varphi(A))$, then $f \circ \varphi$ is $\mathbb{Z}$-prepared on $A$.

We begin with some reductions that apply to any $n \geq 0$. Since $\Pi_{n}(A)$ is bounded, we may work locally in $x$ and thereby assume that $\Pi_{n}(A) \subseteq B_{r}$ and $g \in \mathcal{F}_{n, r}$ for some polyradius $r$ which can be taken to be as small as we wish. Let $\delta>0$ and 
consider the following three sets which cover $A$ :

$$
\begin{aligned}
& A_{\delta}=\{(x, y) \in A:|y|,|g(x) / y|<\delta\} \\
& A_{\delta}^{\prime}=\{(x, y) \in A:|y| \geq \delta\} \\
& A_{\delta}^{\prime \prime}=\{(x, y) \in A:|g(x) / y| \geq \delta\} .
\end{aligned}
$$

Now, $f \circ \varphi$ is $\mathcal{F}$-analytic on $\operatorname{cl}\left(A_{\delta}^{\prime}\right)$. So by Corollary 6.16, $f \circ \varphi$ is $\mathbb{N}$-prepared on $A_{\delta}^{\prime}$ for any $\delta>0$. Also, since $\varphi(A)$ is bounded, there is an $M>0$ such that $\delta \leq|g(x) / y| \leq M$ on $A_{\delta}^{\prime \prime}$. So up to dividing $A_{\delta}^{\prime \prime}$ into smaller sets to account for signs, $y \sim \sigma g(x)$ on $A_{\delta}^{\prime \prime}$ for some $\sigma \in\{-1,1\}$. Hence by Lemma 7.9, $f \circ \varphi$ is $\mathbb{N}$-prepared on $A_{\delta}^{\prime \prime}$ for any $\delta>0$. So it suffices to show that $f \circ \varphi$ is $\mathbb{Z}$-prepared on $A_{\delta}$ for some $\delta>0$. We may therefore assume that $f \in \mathcal{F}_{n+2, s}$ for some $s \geq(r, \delta, \delta)$, and also assume that $0 \in \operatorname{cl}(A)$ and $g(0)=0$, since otherwise $A_{\delta}$ would be empty for sufficiently small $\delta$ and $r$.

If $n=0$, then $f \circ \varphi(x, y)=f(g / y, y)$ for some $g \in \mathbb{R}$. But $g=0$ by our reduction above, so $f \circ \varphi$ is clearly $\mathbb{N}$-prepared on $A_{\delta}$.

So let $n>0$. We need some notation: for any transformation sequence $\mu=$ $\left\langle\mu^{1}, \ldots, \mu^{m}\right\rangle$ and $0 \leq k \leq m$, let $\mu^{\leq k}=\left\langle\mu^{1}, \ldots, \mu^{k}\right\rangle$ and $\mu^{>k}=\left\langle\mu^{k+1}, \ldots, \mu^{m}\right\rangle$. Let $T$ be the transformation tree given by Proposition 7.7. We use the notation given in the proposition: so $T$ respects $y$, and for each $\mu \in T, \mu_{n+1}$ is normal crossings on $\mathcal{B}_{\epsilon(\mu)}$ and $\varphi \circ \mu$ extends to an analytic function $\Phi_{\mu}$ on $\mathcal{B}_{\epsilon(\mu)}$ such that $f \circ \Phi_{\mu}$ is normal crossings. Also, by possibly shrinking $r$ and $\delta$ we may assume that $A_{\delta} \subseteq \bigcup_{\mu \in T} \mu\left(B_{\epsilon(\mu)}\right)$. To finish we simply have to unwind the coordinate transformations in $T$ to show that $f \circ \varphi$ is $\mathbb{Z}$-prepared on $\bigcup_{\mu \in T} \mu\left(B_{\epsilon(\mu)}\right) \cap\{(x, y)$ : $y \neq 0\}$. To do this we show by backwards induction that the following holds for $k=\operatorname{ht}(T), \ldots, 0$ :

For any $\mu=\left\langle\mu^{1}, \ldots, \mu^{m}\right\rangle \in T$ such that $m \geq k, f \circ \varphi \circ \mu^{\leq k}$ is $\mathbb{Z}$-prepared on

$$
V(\mu, k):=\bigcup\left\{\nu^{>k}\left(B_{\epsilon(\nu)}\right): \mu^{\leq k} \subseteq \nu \in T\right\} \cap\left\{(x, y): \mu_{n+1}^{\leq k}(x, y) \neq 0\right\} .
$$

(When $k=0$, this is the statement we want to prove.)

Suppose $k=\operatorname{ht}(T)$. Then $\mu^{\leq k}=\mu$ and $V(\mu, k)=\left\{(x, y) \in B_{\epsilon(\mu)}: \mu_{n+1}(x, y) \neq\right.$ $0\}$. But $f \circ \Phi_{\mu}$ and $\mu_{n+1}$ are normal crossings on $B_{\epsilon(\mu)}$, so $f \circ \varphi \circ \mu$ is clearly $\mathbb{N}$-prepared on $V(\mu, k)$.

Now consider $k<\operatorname{ht}(T)$. Let $\mu=\left\langle\mu^{1}, \ldots, \mu^{m}\right\rangle \in T$ be such that $m \geq k$, and assume that for all $\nu \in T$ such that $\mu^{\leq k} \subseteq \nu, f \circ \varphi \circ \nu \leq k+1$ is $\mathbb{Z}$-prepared on $V(\nu, k+1)$. Put $S=\left\{\nu^{k+1}: \mu^{\leq k} \subseteq \nu \in T\right\}$. By Lemmas 6.6 and 6.7. $f \circ \varphi \circ \mu^{\leq k}$ is $\mathbb{Z}$-prepared on $V(\mu, k) \backslash \operatorname{Cen}(S)$. But $\operatorname{Cen}(S)$ is the (possibly empty) intersection of coordinate subspaces of codimension 1 , so it suffices to show that $\left.f \circ \varphi \circ \mu^{\leq k}(x, y)\right|_{y=0}$ and $\left.f \circ \varphi \circ \mu^{\leq k}(x, y)\right|_{x_{i}=0}$ for $i=1, \ldots, n$ are $\mathbb{Z}$-prepared on $A_{\delta}$. The first function is prepared since it is a term in $x$. For the second function, note that since $\mu^{\leq k}$ is a composition of translations by functions, power substitutions and blow-up substitutions, it follows that

$$
f \circ \varphi \circ \mu^{\leq k}(x, y)=F\left(x, G(x) /\left(L_{1}(x) y+L_{2}(x)\right), L_{1}(x) y+L_{2}(x)\right)
$$

for some $\mathcal{F}$-analytic functions $F, G, L_{1}$ and $L_{2}$. So by applying Lemma 7.8 and the induction hypothesis in $n,\left.f \circ \varphi \circ \mu^{\leq k}(x, y)\right|_{x_{i}=0}$ is $\mathbb{Z}$-prepared on $A_{\delta}$. 
Remark 7.10. For the sake of giving a simpler proof, we used Weierstrass preparation twice in the proof of Proposition 5.1 once to perform the "quantifier elimination" in the proof of Lemma 7.5 (cf. Remark 7.6), and once in the proof of Lemma 7.4. upon which Lemma 7.5 relies. We now sketch an alternate proof of Proposition 5.1 which circumvents this second use of Weierstrass preparation, and thus only uses it to perform the quantifier elimination.

It suffices to study $f(x, g(x) / y, y)$ for values of $(x, y), y \neq 0$, for which $\varphi(x, y)=$ $(x, g(x) / y, y)$ is sufficiently small. Start the proof of Lemma 7.5) in the same way: by performing admissible transformations in $x$, we may assume that $f(0, y, z) \neq 0$. So we may fix $\lambda \in \mathbb{R}$ such that $f(x, y+\lambda z, z)$ is regular in $z$, and hence by Weierstrass preparation, $f(x, y+\lambda z, z)=w(x, y, z) u(x, y, z)$ for some Weierstrass polynomial $w(x, y, z)$ in $z$ and unit $u(x, y, z)$. Let $R(x, t)$ be the resultant in $y$ of $w(x, t, y)$ and $\lambda y^{2}+t y-g(x)=0$, and consider the following sets, where $x, y, g(x) / y, t$ are all complex and sufficiently small:

$$
\begin{aligned}
Z_{1} & =\{(x, y): y \neq 0, f(x, g(x) / y, y)=0\}, \\
Z_{2} & =\left\{(x, y, t): y \neq 0, f(x, t+\lambda y, y)=\lambda y^{2}+t y-g(x)=0\right\}, \\
Z_{3} & =\left\{(x, t): \exists y \text { s.t. }(x, y, t) \in Z_{2}\right\}, \\
Z_{4} & =\{(x, t): R(x, t)=0\} .
\end{aligned}
$$

We have two surjections, $Z_{1} \rightarrow Z_{2}:(x, y) \mapsto(x, y, g(x) / y-\lambda y)$ and $Z_{2} \rightarrow Z_{3}$ : $(x, y, t) \mapsto(x, t)$. The first is injective but the second is 2-to- 1 , so $Z_{3}$ is the image of $Z_{1}$ under the 2-to-1 mapping $(x, y) \mapsto(x, g(x) / y-\lambda y)$. Since $Z_{3} \subseteq Z_{4}$, we get that $f(x, g(x) / y, y)=0$ implies $R(x, g(x) / y-\lambda y)=0$.

Now the two proofs diverge. The two "inverse" maps of $(x, y) \mapsto(x, g(x) / y-\lambda y)$ are

$$
Q_{\sigma}(x, y)=\left(x, \frac{-y+\sigma \sqrt{y^{2}+4 \lambda g(x)}}{2 \lambda}\right)
$$

for $\sigma \in\{1,-1\}$, so $f \circ \varphi \circ Q_{\sigma}(x, y)=0$ implies $R(x, y)=0$. Because

$$
\varphi \circ Q_{\sigma}(x, y)=\left(x, \frac{y+\sigma \sqrt{y^{2}+4 \lambda g(x)}}{2}, \frac{-y+\sigma \sqrt{y^{2}+4 \lambda g(x)}}{2 \lambda}\right),
$$

$f \circ \varphi \circ Q_{\sigma}$ is not analytic simply because $\sqrt{y^{2}+4 \lambda g(x)}$ is not analytic. Put $D(x, y)=$ $y^{2}+4 \lambda g(x)$. Now, there is a full transformation tree $T$ in $(x, y)$ which respects $y$ and transforms $R(x, y) \cdot D(x, y)$ to normal crossings. So for any $\mu \in T$, both $R \circ \mu$ and $D \circ \mu$ are normal crossings. Put $D \circ \mu(x, y)=x^{\alpha} y^{d} u(x, y)$, and note that $d \geq 2$ because the order of a function in a given variable cannot drop by performing admissible transformations. By possibly extending $\mu$ by power substitutions in $x$, we may also assume that $\alpha_{i} \geq 2$ for $i=1, \ldots, n$. Therefore $f \circ \varphi \circ Q_{\sigma} \circ \mu$ is analytic, and its complex zero set lies in the complex zero set of $R \circ \mu$, so $f \circ \varphi \circ Q_{\sigma} \circ \mu$ is normal crossings.

We already know how to unwind $\mathcal{F}$-admissible transformations, so it suffices to see that we can also unwind the transformations $Q_{\sigma}, \sigma \in\{1,-1\}$. Namely, it suffices to show that given a cylinder $C \subseteq \mathbb{R}^{n+1}, Q_{\sigma}(C)$ is a finite union of cylinders, and that given a function $F(x, y)$, if $F \circ Q_{\sigma}(x, y)$ is $\mathbb{Z}$-prepared, then so is $F(x, y)$.

Suppose that $C=\{(x, y) \in B \times \mathbb{R}: s(x)<y<t(x)\}$; we may assume that $y \neq 0$ for $(x, y) \in C$. Then $Q_{\sigma}(C)=\left\{(x, y):\left(Q_{\sigma}\right)^{-1}(x, y) \in C\right\}=\{(x, y) \in$ 
$B \times \mathbb{R}: s(x)<g(x) / y-\lambda y<t(x)\}$, which is a finite union of cylinders because $\lambda y^{2}+s(x) y-g(x)$ and $\lambda y^{2}+t(x) y-g(x)$ are easily prepared.

Now suppose that $F \circ Q_{\sigma}(x, y)=a(x)(y-\theta(x))^{d} u(x, y-\theta(x))$, and apply $\left(Q_{\sigma}\right)^{-1}(x, y)=(x, g(x) / y-\lambda y)$ to get

$$
F(x, y)=a(x)(g(x) / y-\lambda y-\theta(x))^{d} u(x, g(x) / y-\lambda y-\theta(x)) .
$$

But $g(x) / y-\lambda y-\theta(x)=\left(\lambda y^{2}-\theta(x) y-g(x)\right) / y$. The numerator is easily $\mathbb{N}$ prepared, and the denominator is trivially $\mathbb{N}$-prepared, so the result follows from simultaneous preparation.

\section{Proof of the preparation theorem For a General Field $K$}

This section completes the proof of the Main Theorem by showing the following.

Proposition 8.1. Let $\mathcal{F}$ be an analytic system of germs closed under composition and implicit functions, and let $K$ be a subfield of the field of exponents of $\mathcal{F}$ (which is indeed a field by our hypotheses). If every $\mathcal{L}_{\mathcal{F}}^{\mathbb{Q}}$-definable function is $\mathcal{L}_{\mathcal{F}}^{\mathbb{Q}}$-prepared, then every $\mathcal{L}_{\mathcal{F}}^{K}$-definable function is $\mathcal{L}_{\mathcal{F}}^{K}$-prepared.

Proof. To show this, it suffices to show that the three claims in the proof of the preparation theorem for $K=\mathbb{Q}$ found in Section 5 are true for a general $K$. It is clear that if $f(x, y)$ is $\mathcal{L}_{\mathcal{F}}^{K}$-prepared, then $f(x, y)^{r}$ is $\mathcal{L}_{\mathcal{F}}^{K}$-prepared for any $r \in K$. The rest of the content of the three claims are special cases of the following statement which we shall prove:

Suppose that $f: \mathbb{R}^{m} \rightarrow \mathbb{R}$ is $\mathcal{L}_{\mathcal{F}}^{\mathbb{Q}}$-definable and that $g=\left(g_{1}, \ldots, g_{m}\right):$ $\mathbb{R}^{n+1} \rightarrow \mathbb{R}^{m}$ is such that each $g_{i}$ is $\mathcal{L}_{\mathcal{F}}^{K}$-prepared. Then $f \circ g$ is $\mathcal{L}_{\mathcal{F}}^{K}$-prepared.

By simultaneous preparation, it suffices to show that $f \circ g$ is $\mathcal{L}_{\mathcal{F}}^{K}$-prepared on $A \subseteq \mathbb{R}^{n+1}$, where $A$ is a fat $\mathcal{L}_{\mathcal{F}}^{K}$-cylinder such that for $i=1, \ldots, m$,

$$
g_{i}(x, y)=b_{i}(x)|y-\theta(x)|^{r_{i}} u_{i}(x, y-\theta(x))
$$

on $A$ for some $\mathcal{L}_{\mathcal{F}}^{K}$-terms $b_{i}$ and $\theta, r_{i} \in K$ and $\mathcal{L}_{\mathcal{F}}^{K}$-special unit $u_{i}$. We could always work in the variables $\pm(y-\theta(x))$, so to simplify notation we assume that $\theta=0$ and that $y>0$ on $A$. We may therefore rewrite $f \circ g(x, y)$ as $F \circ \varphi(x, y)$, where $\varphi: A \rightarrow \mathbb{R}^{l+k}$ is an $\mathcal{F}$-analytic function of the form $\varphi(x, y)=\left(a(x), y^{r_{1}}, \ldots, y^{r_{k}}\right)$ for some $l$-tuple of $\mathcal{L}_{\mathcal{F}}^{K}$-terms $a(x)$ and numbers $r_{1}, \ldots, r_{k} \in K$, and $F: \mathbb{R}^{l+k} \rightarrow \mathbb{R}$ is $\mathcal{L}_{\mathcal{F}}^{\mathbb{Q}}$-definable. By possibly adjusting the definition of $F$, we can take $r_{1}, \ldots, r_{k}$ to be linearly independent over the rationals.

Lemma 8.2. Let $\varphi$ and $F$ be as in the discussion above.

(i) If $F \circ \varphi(x, y)=G(b(x), y)$ on $A$ for some $p$-tuple of $\mathcal{L}_{\mathcal{F}}^{K}$-terms $b(x)$ and $\mathcal{L}_{\mathcal{F}}^{\mathbb{Q}}$-definable function $G: \mathbb{R}^{p+1} \rightarrow \mathbb{R}$, then $F \circ \varphi$ is $\mathcal{L}_{\mathcal{F}}^{K}$-prepared on $A$.

(ii) If $y \sim \theta(x)$ on $A$ for some $\mathcal{L}_{\mathcal{F}}^{K}$-term $\theta(x)$, then there is a finite $\operatorname{cover} \mathcal{C}$ of $A$ by $\mathcal{L}_{\mathcal{F}}^{K}$-cylinders such that on each $C \in \mathcal{C}, F \circ \varphi$ satisfies the hypothesis of (i).

Proof. To prove (i) simply $\mathcal{L}_{\mathcal{F}}^{\mathbb{Q}}$-prepare $G\left(z_{1}, \ldots, z_{p}, y\right)$. This induces an $\mathcal{L}_{\mathcal{F}}^{K}$-preparation of $G(b(x), y)$ on $A$.

To prove (ii) proceed as in the proof of Lemma 7.9 and use the fact $K$ is a subfield of the field of exponents of $\mathcal{F}$. Namely, first reduce to the case that 
$|(y-\theta(x)) / \theta(x)| \leq 1 / 2$ on $A$, and then write $y^{r_{i}}=\theta(x)^{r_{i}}(1+(y-\theta(x)) / \theta(x))^{r_{i}}$ for each $i$.

By (i) in the previous lemma and the reductions above, it suffices to show the following:

$\left(\mathrm{I}_{k}\right)$ Let $A \subseteq \mathbb{R}^{n} \times(0, \infty)$ be a fat $\mathcal{L}_{\mathcal{F}}^{K}$-cylinder, let $\varphi: A \rightarrow \mathbb{R}^{l+k}$ be an $\mathcal{F}$-analytic function of the form $\varphi(x, y)=\left(a(x), y^{r_{1}}, \ldots, y^{r_{k}}\right)$ for an $l$-tuple of $\mathcal{L}_{\mathcal{F}}^{K}$-terms $a(x)$ and numbers $r_{1}, \ldots, r_{k} \in K$ which are linearly independent over the rationals, and let $F: \mathbb{R}^{l+k} \rightarrow \mathbb{R}$ be $\mathcal{L}_{\mathcal{F}}^{\mathbb{Q}}$-definable. Then there is a finite cover $\mathcal{C}$ of $A$ by $\mathcal{L}_{\mathcal{F}}^{K}$-cylinders such that for each $C \in \mathcal{C}$ either

(a) $F \circ \varphi(x, y)=G(b(x), y)$ on $C$ for some $p$-tuple of $\mathcal{L}_{\mathcal{F}}^{K}$-terms $b(x)$ and $\mathcal{L}_{\mathcal{F}}^{\mathbb{Q}}$-definable function $G: \mathbb{R}^{p+1} \rightarrow \mathbb{R}$, or

(b) $F \circ \varphi(x, y)=b(x) y^{s} u(x, y)$ on $C$ for some $\mathcal{L}_{\mathcal{F}}^{K}$-term $b(x), \mathcal{L}_{\mathcal{F}}^{K}$-special unit $u(x, y)$ and $s \in \operatorname{span}_{\mathbb{Q}}\left(r_{1}, \ldots, r_{k}\right)$.

Given $0 \leq i \leq k$ and a set $D \subseteq \mathbb{R}^{l+i}$, and given $a(x)$ and $r_{1}, \ldots, r_{k}$ as in $\left(\mathrm{I}_{k}\right)$, let

$$
D_{A}:=\left\{(x, y) \in A:\left(a(x), y^{r_{1}}, \ldots, y^{r_{i}}\right) \in D\right\} .
$$

We prove $\left(\mathrm{I}_{k}\right)$ by induction on $k$, and for the induction to go through we need to also induct on the following statement:

$\left(\mathrm{II}_{k}\right)$ Let $A$ and $\varphi: A \rightarrow \mathbb{R}^{l+k}$ be as in $\left(\mathrm{I}_{k}\right)$. Then for any $\mathcal{L}_{\mathcal{F}}^{\mathbb{Q}}$-definable set $C \subseteq \mathbb{R}^{l+k}, C_{A}$ is a finite union of $\mathcal{L}_{\mathcal{F}}^{K}$-cylinders.

Both $\left(\mathrm{I}_{0}\right)$ and $\left(\mathrm{II}_{0}\right)$ are obvious, so let $k>0$ and assume $\left(\mathrm{I}_{k-1}\right)$ and $\left(\mathrm{II}_{k-1}\right)$.

Proof of $\left(\mathrm{I}_{k}\right)$. If $k>1$, then we can reorder $r_{1}, \ldots, r_{k}$ to ensure that $r_{k} \notin \mathbb{Q}$. If $k=1$ and $r_{1} \in \mathbb{Q}$, then we are in case (a). We may therefore assume that $r_{k}$ is irrational.

Fix a tight $\mathcal{L}_{\mathcal{F}}^{\mathbb{Q}}$-preparation of $F\left(z, y^{\prime}, y_{k}\right)$ in the variable $y_{k}$, where $z=\left(z_{1}, \ldots, z_{l}\right)$ and $y^{\prime}=\left(y_{1}, \ldots, y_{k-1}\right)$. By $\left(\mathrm{II}_{k-1}\right)$ we know that for any $\mathcal{L}_{\mathcal{F}}^{\mathbb{Q}}$-cylinder $C$ given by this preparation, say with base $D, D_{A}$ is a finite union of $\mathcal{L}_{\mathcal{F}}^{K}$-cylinders. Therefore by partitioning $A$ into finitely many smaller $\mathcal{L}_{\mathcal{F}}^{K}$-cylinders and considering each of these cylinders separately, we may reduce to the following situation: there is finite collection $\mathcal{C}$ of $\mathcal{L}_{\mathcal{F}}^{\mathbb{Q}}$-cylinders in $\mathbb{R}^{l+k}$ such that

(i) all the $C \in \mathcal{C}$ have the same base $D \subseteq \mathbb{R}^{l+k-1}$;

(ii) $D_{A}=A$;

(iii) $A=\bigcup_{C \in \mathcal{C}} C_{A}$;

(iv) there are $\mathcal{L}_{\mathcal{F}}^{\mathbb{Q}}$-terms $t_{1}\left(z, y^{\prime}\right), \ldots, t_{p}\left(z, y^{\prime}\right)$ which are $\mathcal{F}$-analytic on $D$, are such that $-\infty=t_{0}\left(z, y^{\prime}\right)<\cdots<t_{p+1}\left(z, y^{\prime}\right)=\infty$ on $D$, and are such that $\mathcal{C}=\left\{C^{0,1}, C^{1}, \ldots, C^{p}, C^{p, p+1}\right\}$, where

$$
\begin{aligned}
C^{i} & =\left\{\left(z, y^{\prime}, y_{k}\right) \in D \times \mathbb{R}: y_{k}=t_{i}\left(z, y^{\prime}\right)\right\}, \\
C^{i, i+1} & =\left\{\left(z, y^{\prime}, y_{k}\right) \in D \times \mathbb{R}: t_{i}\left(z, y^{\prime}\right)<y_{k}<t_{i+1}\left(z, y^{\prime}\right)\right\} ;
\end{aligned}
$$

(v) $F\left(z, y^{\prime}, y_{k}\right)$ is of a tight $\mathcal{L}_{\mathcal{F}}^{\mathbb{Q}}$-prepared form on each $C \in \mathcal{C}$. 
Because $D$ is quantifier free $\mathcal{L}_{\mathcal{F}}^{K}$-definable, the proof of Lemma 3.7(ii) shows that $D$ is the union of finitely many $\mathcal{L}_{\mathcal{F}}^{K}$-term cells, so we may further assume that $D$ is an $\mathcal{L}_{\mathcal{F}}^{K}$-term cell.

Now, the idea of the proof is to use the $\mathcal{L}_{\mathcal{F}}^{\mathbb{Q}}$-preparation of $F\left(z, y^{\prime}, y_{k}\right)$ on $C$ to obtain an $\mathcal{L}_{\mathcal{F}}^{K}$-preparation of $F\left(a(x), y^{r_{1}}, \ldots, y^{r_{k}}\right)$ on $C_{A}$ for each $C \in \mathcal{C}$. But it is not at all clear that $C_{A}$ is a finite union of $\mathcal{L}_{\mathcal{F}}^{K}$-cylinders, as it in fact must be by the proposition we are trying to prove. This problem can be circumvented with the help of the following claim.

Claim. By possibly dividing $A$ into smaller $\mathcal{L}_{\mathcal{F}}^{K}$-cylinders, we may assume that for each $i=1, \ldots, p$ there is an $\mathcal{L}_{\mathcal{F}}^{K}$-term $\psi_{i}(x)$ which is $\mathcal{F}$-analytic on $\Pi_{n}(A)$ and such that $y \sim \psi_{i}(x)$ on $C_{A}^{i}$.

Because $A=D_{A}, C_{A}^{i}=\left\{(x, y) \in A: y^{r_{k}}=t_{i}\left(a(x), y^{r_{1}}, \ldots, y^{r_{k-1}}\right)\right\}$. By applying $\left(\mathrm{I}_{k-1}\right)$ to $t_{i}\left(a(x), y^{r_{1}}, \ldots, y^{r_{k-1}}\right)$ and dividing $A$ into smaller $\mathcal{L}_{\mathcal{F}}^{K}$-cylinders accordingly, we may assume that $t_{i}\left(a(x), y^{r_{1}}, \ldots, y^{r_{k-1}}\right)=b(x)|y-\theta(x)|^{s} u(x, y-\theta(x))$ on $A$, where $b$ and $\theta$ are $\mathcal{L}_{\mathcal{F}}^{K}$-terms, $u$ is an $\mathcal{L}_{\mathcal{F}}^{K}$-special unit and the following holds:

(i) if $\theta \neq 0$, then $s \in \mathbb{Q}$ and $y \sim \theta(x)$ on $A$;

(ii) if $\theta=0$, then either $s \in \mathbb{Q}$ or $s \in \operatorname{span}_{\mathbb{Q}}\left\{r_{1}, \ldots, r_{k-1}\right\}$; hence $y \sim(b(x))^{1 /\left(r_{k}-s\right)}$ on $C_{A}^{i}$.

This proves the claim.

Fix $\epsilon_{i}>1$ such that $E^{i}=\left\{(x, y) \in A: \epsilon_{i}^{-1} \leq y / \psi_{i}(x) \leq \epsilon_{i}\right\}$ contains $C_{A}^{i}$. By possibly dividing $D$ into smaller $\mathcal{L}_{\mathcal{F}}^{K}$-term cells, we may assume that the sets $E^{i}$ are all $\mathcal{L}_{\mathcal{F}}^{K}$-term cells with base $D$, and that $A \backslash \bigcup_{i=1}^{p} E^{i}$ has finitely many connected components, each of which is an $\mathcal{L}_{\mathcal{F}}^{K}$-term cell with base $D$. We are in case (a) on each $E^{i}$, so it suffices to consider a component $E^{\prime}$ of $A \backslash \bigcup_{i=1}^{p} E^{i}$. Because $\varphi$ is continuous, $\varphi\left(E^{\prime}\right)$ is connected. Also, $\varphi\left(E^{\prime}\right) \cap C^{i}=\emptyset$ for all $i=1, \ldots, p$, so $\varphi\left(E^{\prime}\right) \subset \bigcup_{i=1}^{p} C^{i, i+1}$. But the $C^{i, i+1}$ 's are exactly the connected components of $\bigcup_{i=1}^{p} C^{i, i+1}$, so $\varphi\left(E^{\prime}\right) \subseteq C^{i, i+1}$ for some $i$. Therefore by simply taking $A$ to be the cell $E^{\prime}$, we have reduced to the following situation: $\mathcal{C}$ consists of a single fat $\mathcal{L}_{\mathcal{F}}^{\mathbb{Q}}$-cylinder. Therefore on $A$,

$$
\begin{array}{r}
F\left(a(x), y^{r_{1}}, \ldots, y^{r_{k}}\right) \\
=b\left(a(x), y^{r_{1}}, \ldots, y^{r_{k-1}}\right)\left|y^{r_{k}}-\theta\left(a(x), y^{r_{1}}, \ldots, y^{r_{k-1}}\right)\right|^{q} \\
\quad u\left(a(x), y^{r_{1}}, \ldots, y^{r_{k-1}}, y^{r_{k}}-\theta\left(a(x), y^{r_{1}}, \ldots, y^{r_{k-1}}\right)\right)
\end{array}
$$

for some $\mathcal{L}_{\mathcal{F}}^{\mathbb{Q}}$-terms $b\left(z, y^{\prime}\right)$ and $\theta\left(z, y^{\prime}\right), q \in \mathbb{Q}$ and $\mathcal{L}_{\mathcal{F}}^{\mathbb{Q}}$-special unit $u$. If $\theta \neq 0$, then $y^{r_{k}} \sim \theta\left(a(x), y^{r_{1}}, \ldots, y^{r_{k-1}}\right)$, so by applying $\left(\mathrm{I}_{k-1}\right)$ to $\theta\left(a(x), y^{r_{1}}, \ldots, y^{r_{k-1}}\right)$ and proceeding as in the proof of the Claim, we reduce to case (a). We may therefore assume that $\theta=0$. But then by applying $\left(\mathrm{I}_{k-1}\right)$ to $b\left(a(x), y^{r_{1}}, \ldots, y^{r_{k-1}}\right)$, we either reduce to case (a) in a similar manner or else to the case that $b\left(a(x), y^{r_{1}}, \ldots, y^{r_{k-1}}\right)=$ $c(x) y^{s} v(x, y)$ on $A$ for some $\mathcal{L}_{\mathcal{F}}^{K}$-term $c(x), s \in \operatorname{span}\left\{r_{1}, \ldots, r_{k}\right\}$ and $\mathcal{L}_{\mathcal{F}}^{K}$-unit $v(x, y)$. We are then in case (b).

Proof of $\left(\mathrm{II}_{k}\right)$. It suffices to consider an $\mathcal{L}_{\mathcal{F}}^{\mathbb{Q}}$-cylinder $C \subseteq \mathbb{R}^{l+k}$ and show that $C_{A}$ is a finite union of $\mathcal{L}_{\mathcal{F}}^{K}$-cylinders. So $C=\left\{\left(z, y^{\prime}, y_{k}\right) \in D \times \mathbb{R}: \varphi\left(z, y^{\prime}, y_{k}\right)\right\}$ for some formula $\varphi\left(z, y^{\prime}, y_{k}\right)$ of the form $y_{k}=t\left(z, y^{\prime}\right), s\left(z, y^{\prime}\right)<y_{k}<t\left(z, y^{\prime}\right), s\left(z, y^{\prime}\right)<y_{k}$ or $y_{k}<t\left(z, y^{\prime}\right)$ for $\mathcal{L}_{\mathcal{F}}^{\mathbb{Q}}$-terms $s$ and $t$. By $\left(\mathrm{II}_{k-1}\right)$ we may divide $A$ into smaller 
$\mathcal{L}_{\mathcal{F}}^{K}$-cylinders to assume that $A=D_{A}$. So $C_{A}=\left\{(x, y) \in A: \varphi\left(a(x), y^{r_{1}}, \ldots, y^{r_{k}}\right)\right\}$. By $\left(\mathrm{I}_{k}\right)$ the functions $y^{r_{k}}-s\left(a(x), y^{r_{1}}, \ldots, y^{r_{k-1}}\right)$ and $y^{r_{k}}-t\left(a(x), y^{r_{1}}, \ldots, y^{r_{k-1}}\right)$ are $\mathcal{L}_{\mathcal{F}}^{K}$-prepared on $A$, so by further dividing $A$ into smaller $\mathcal{L}_{\mathcal{F}}^{K}$-cylinders, we may assume that these functions have constant signs on $A$. Then $C_{A}$ is either $A$ or is empty.

This completes the proof of Proposition 8.1 .

\section{Appendix: Various closure properties OF ANALYTIC SYSTEMS OF GERMS}

We now discuss various closure properties one might want to impose upon an analytic system of germs. We break the discussion down into two parts:

1. We compare Weierstrass systems with Van den Dries's notion of a "convergent Weierstrass system" [22 and discuss the question of whether Weierstrass preparation is closed under definability.

2. We discuss closure properties relating to Lion and Rolin's splitting technique and their globally subanalytic integration theorem [14.

9.1. Closure under definability. The Weierstrass division theorem states that for all $f, g \in \mathcal{O}_{n+1}$, if $f(x, y)$ is regular in $y$ of order $d$, then there exist unique $q \in \mathcal{O}_{n+1}$ and $r_{0}, \ldots, r_{d-1} \in \mathcal{O}_{n}$ such that

$$
g(x, y)=f(x, y) q(x, y)+\sum_{i=0}^{d-1} r_{i}(x) y^{i} .
$$

An analytic system of germs $\mathcal{F}$ is "closed under Weierstrass division" if for all $f, g \in \mathcal{F}_{n+1}$ such that $f(x, y)$ is regular in $y$ of order $d$, if $q$ and $r_{0}, \ldots, r_{d-1}$ are the analytic germs given by (9.1), then $q \in \mathcal{F}_{n+1}$ and $r_{0}, \ldots, r_{d-1} \in \mathcal{F}_{n}$. If an analytic system of germs is closed under Weierstrass division, it is also closed under Weierstrass preparation: indeed, if $f(x, y)$ is regular in $y$ of order $d$, to Weierstrass prepare $f$ simply Weierstrass divide $y^{d}$ by $f$.

In Definition 2.1 we defined a Weierstrass system to be an analytic system of germs closed under composition and Weierstrass preparation. An analytic system of germs closed under composition and Weierstrass division is exactly a "convergent Weierstrass system" in the sense of Van den Dries [22] (cf. Denef and Lipshitz [7, 1.3 Remarks]), disregarding the merely cosmetic difference that [22] states its definitions in terms of convergent power series rather than real analytic germs. To compare the two concepts we use the following notation: for any $\mathcal{G} \subseteq \mathcal{O}$ closed under local translation, let $\mathrm{WP}(\mathcal{G})$ denote the smallest analytic system of germs containing $\mathcal{G}$ which is closed under composition and Weierstrass preparation, and likewise let $\mathrm{WD}(\mathcal{G})$ denote the smallest analytic system of germs containing $\mathcal{G}$ which is closed under composition and Weierstrass division. To construct $\mathrm{WP}(\mathcal{G})$ or $\operatorname{WD}(\mathcal{G})$ from $\mathcal{G}$, first consider the collection of all functions $g: U \rightarrow \mathbb{R}$, with $U$ a neighborhood of the origin, such that $\mathcal{G}$ contains the germ of $g$ at each point of $U$, along with the collection of all real constants and all coordinate projection functions $x \mapsto$ $x_{i}$. Then close under the operations of addition, multiplication, composition, and either Weierstrass preparation or Weierstrass division, respectively. One obtains a collection of analytic functions defined in neighborhoods of the origin in $\mathbb{R}^{n}$, for all $n \in \mathbb{N}$, and $\operatorname{WP}(\mathcal{G})$ or $\operatorname{WD}(\mathcal{G})$ is the collection of germs at the origin of this collection of functions. (From this one sees that the assumption that $\mathcal{G}$ is closed 
under local translation is needed for $\mathrm{WP}(\mathcal{G})$ and $\mathrm{WD}(\mathcal{G})$ to be well defined. Indeed, since $\mathrm{WP}(\mathcal{G})$ and $\mathrm{WD}(\mathcal{G})$ are by definition closed under local translation, for each germ $g$ in $\mathcal{G}$ there must be a representative $g: U \rightarrow \mathbb{R}$ such that $\mathrm{WP}(\mathcal{G})$ and $\mathrm{WD}(\mathcal{G})$ contain the germ of $g$ at each point of $U$. If $\mathcal{G}$ was not closed under local translation, we could choose $U$ to be arbitrarily small. But the smaller we choose these $U$ 's, the smaller the generated system of germs would be, so there would be no smallest collection of germs with the desired closure properties.)

Of course $\mathrm{WP}(\mathcal{G}) \subseteq \mathrm{WD}(\mathcal{G})$, but in spite of the fact that the Weierstrass preparation and division theorems are commonly treated as being equivalent, I do not know if equality holds. Namely, is every Weierstrass system closed under Weierstrass division? I am primarily interested in the following weaker form of this question.

Question 1. Is every member of $\operatorname{WD}(\mathcal{G})$ necessarily the germ of some function definable in $\mathbb{R}_{\mathrm{WP}(\mathcal{G})}$ ?

This question may relate to the following.

Question 2. For every analytic system of germs $\mathcal{F}$ which is closed under composition, is every member of $\operatorname{WP}(\mathcal{F})$ the germ of some function definable in $\mathbb{R}_{\mathcal{F}}$ ?

The potential relationship between these two questions lies in the following proposition which I learned from Chris Miller, whose proof is due mainly to Van den Dries to the best of my knowledge.

Proposition 9.1. (i) If $\mathcal{F}=\mathrm{WD}(\mathcal{F})$, then $\mathcal{F}$ is "closed under complexification" in the following sense: for each $f \in \mathcal{F}_{n}$ there exist $R, C \in \mathcal{F}_{2 n}$ such that $f(x+i y)=R(x, y)+i C(x, y)$, where $i=\sqrt{-1}$.

(ii) If $\mathcal{F}$ is an analytic system of germs closed under local translation and complexification, then every member of $\operatorname{WD}(\mathcal{F})$ is the germ of some function definable in $\mathbb{R}_{\mathcal{F}}$.

(iii) Question 2 has a negative answer if $\operatorname{WP}(\mathcal{F})$ is replaced with $\operatorname{WD}(\mathcal{F})$.

Proof. To prove (i) consider variables $x=\left(x_{1}, \ldots, x_{n}\right), y=\left(y_{1}, \ldots, y_{n}\right), w$ and $z$. By Weierstrass division there exist $r_{0}, r_{1} \in \mathcal{F}_{2 n+1}$ and $q \in \mathcal{F}_{2 n+2}$ such that

$$
f(x+z y)=\left(z^{2}+w^{2}\right) q(x, y, w, z)+r_{0}(x, y, w)+r_{1}(x, y, w) z .
$$

Pass to representatives of these germs, which we shall also denote by $f, q, r_{0}$ and $r_{1}$. For a sufficiently small $\epsilon>0$ we may apply the substitution $(x, y, w, z) \mapsto$ $(x, y / \epsilon, \epsilon, i \epsilon)$ to (9.2) to obtain

$$
f(x+i y)=r_{0}(x, y / \epsilon, \epsilon)+i \epsilon r_{1}(x, y / \epsilon, \epsilon) .
$$

So $R(x, y)$ and $C(x, y)$ are in $\mathcal{F}_{2 n}$ since they are the germs at 0 of $r_{0}(x, y \epsilon, \epsilon)$ and $\epsilon r_{1}(x, y / \epsilon, \epsilon)$, respectively. This proves (i).

Statement (ii) follows from 22. Statement (iii) follows from (i) and Bianconi 1]. Indeed, if we let $\mathcal{F}$ be the smallest analytic system of germs closed under composition and containing the germ at the origin of the exponential function, then $\sin x$ is in $\operatorname{WD}(\mathcal{F})$ but is not the germ of any function definable in $\mathbb{R}_{\mathcal{F}}$. (Likewise with the roles of $\exp x$ and $\sin x$ reversed.)

I would like to discuss Question 1. Because every naturally occurring Weierstrass system of which I am aware (namely $\mathcal{A}, \mathcal{D}$ and $\mathcal{O}$ ) is closed under Weierstrass 
division, this question is not currently of practical interest to me. Nevertheless, it is of theoretical interest because if Question 1 has a positive answer, Question 2 would have a negative answer by Proposition 9.1(iii). (Although, I actually suspect that both questions have negative answers but have no proof.) I am only aware of two classical proofs of the Weierstrass division theorem from the Weierstrass preparation theorem, and both use additional closure properties of analytic functions other than just closure under composition.

Grauert and Remmert [11, pg. 43] prove the division theorem by using the preparation theorem, along with multiplication of Laurent series. The latter operation uses a certain partitioning of terms very reminiscent of Lion and Rolin's splitting technique. The whole purpose of this paper is to avoid such techniques, as it seems highly doubtful that Weierstrass systems in general should be closed under such splitting operations.

A more well-known proof of the division theorem uses the preparation theorem and contour integration (see Gunning and Rossi [12]). This is bad for two reasons. First, since contour integrals involve holomorphic functions on open subsets of $\mathbb{C}^{n}$, to use this proof our Weierstrass system $\mathcal{F}$ must be closed under complexifications, an assumption we cannot make without risk of circularity. Second, to compute contour integrals and stay within $\mathcal{F}$, the system $\mathcal{F}$ must be "closed under antidifferentiation": namely, for every $f(x, y) \in \mathcal{F}_{n+1}$ there exists $F \in \mathcal{F}_{n+1}$ such that $\frac{\partial F}{\partial y}=f$. But this property is not generally true of Weierstrass systems since it fails for both $\mathcal{A}$ and $\mathcal{D}$.

Proof. The failure for $\mathcal{A}$ is easy: $(1+x)^{-1} \in \mathcal{A}_{1}$ but $\ln (1+x) \notin \mathcal{A}_{1}$. The failure for $\mathcal{D}$ follows from [15, Proposition 6.3], which shows that $\sum_{k=0}^{\infty} x^{k} / k$ ! and $\sum_{k=0}^{\infty} x^{k^{2}}$ are differentially algebraic but their Hadamard product $\sum_{k=0}^{\infty} x^{k^{2}} /\left(k^{2}\right)$ ! is not. Therefore Proposition 9.2 below shows that $\mathcal{D}$ is not closed under antidifferentiation.

In addition to Question 2, another reason to discuss the potential difference between the Weierstrass preparation and division theorems is that not only does the Lion-Rolin preparation theorem hold for Weierstrass systems, but the Weierstrass preparation theorem is also sufficient to obtain Denef and Van den Dries's quantifier elimination for the expansion of $\mathbb{R}_{\mathcal{F}}$ by division (cf. [8] and [22]). They never use Weierstrass division directly, but rather just two of its consequences:

(i) faithful flatness (i.e., [22, 1.3 Lemma]) in the proof of [8, 4.12 Lemma];

(ii) Weierstrass preparation in the proof of [8, 4.13 Local Basic Lemma].

But, faithful flatness is not needed because in Denef and Van den Dries's argument, 8, 4.12 Lemma] can be replaced with a suitable variant of Lemma 6.17 simply replace our local resolution procedure involving local blowings-up and power substitutions with the procedure of 22 involving only local blowings-up so that only division, and not rational powers, are needed to unwind its coordinate transformations.

9.2. Lion and Rolin's splitting technique. Since the properties of closure under complexification and closure under antidifferentiation have already been mentioned, to conclude I would like to point out a simple relationship between them and Lion and Rolin's splitting technique. 
Proposition 9.2. Let $\mathcal{F}$ be an analytic system of germs closed under composition and complexification.

(i) If $\mathcal{F}$ is closed under antidifferentiation and multiplicative inverses, then $\mathcal{F}$ is "closed under Hadamard products": namely, if $f(x, z)=\sum_{\alpha \in \mathbb{N} m} f_{\alpha}(x) z^{\alpha}$ and $g(x, z)=\sum_{\alpha \in \mathbb{N} m} g_{\alpha}(x) z^{\alpha}$ are in $\mathcal{F}_{n+m}$, then their Hadamard product in $z, \sum_{\alpha \in \mathbb{N}^{m}} f_{\alpha}(x) g_{\alpha}(x) z^{\alpha}$, is in $\mathcal{F}_{n+m}$.

(ii) If $\mathcal{F}$ is closed under Hadamard products and contains $\ln \left(1-x_{1}\right)$, then $\mathcal{F}$ is closed under antidifferentiation.

Note that $\ln \left(1-x_{1}\right) \in \mathcal{F}_{1}$ if $\mathcal{F}$ contains the exponential function and is closed under implicit functions. Also, if $\mathcal{F}$ is closed under composition, multiplicative inverses, complexification and antidifferentiation, then by the use of classical contour integral representations it is not hard to show (in the stated order) that $\mathcal{F}$ is closed under differentiation, Weierstrass preparation and finally Weierstrass division.

Proof of Proposition 9.2. To prove (i) we use the variables $x=\left(x_{1}, \ldots, x_{n}\right), y=$ $\left(y_{1}, \ldots, y_{m}\right), z=\left(z_{1}, \ldots, z_{m}\right)$, and $w=\left(w_{1}, \ldots, w_{m}\right)$. Let $C$ be a sufficiently small square about the origin in $\mathbb{C}$, viewed as a positively oriented contour. Then by the residue theorem,

$$
\begin{aligned}
& \sum_{\alpha \in \mathbb{N}^{m}} f_{\alpha}(x) y^{\alpha} z^{\alpha} \\
& =\frac{1}{(2 \pi i)^{m}} \int_{C} \cdots \int_{C} \frac{f\left(x, y_{1} w_{1}, \ldots, y_{m} w_{m}\right) g\left(x, z_{1} / w_{1}, \ldots, z_{m} / w_{m}\right)}{w_{1} \cdots w_{m}} d w_{1} \cdots d w_{m},
\end{aligned}
$$

which is in $\mathcal{F}$ by our assumptions. Substituting $(y, z) \mapsto(y / \epsilon, \epsilon)$ for sufficiently small $\epsilon \in(0, \infty)^{m}$ proves (i).

Statement (ii) follows from the fact that an antiderivative of $f(x, y) \in \mathcal{F}_{n+1}$ in $y$ can be expressed as the Hadamard product in $y$ of $y f(x, y)$ with $-\ln (1-y)$.

Proposition 9.3 (Lion and Rolin splitting). Suppose that $\mathcal{F}$ is closed under composition, multiplicative inverses, complexifications and antidifferentiation, and let $f(x, y, z)$ be $\mathcal{F}$-analytic at the origin in $\mathbb{R}^{n} \times \mathbb{R} \times \mathbb{R}$. Then for sufficiently small $x$, $y / z$ and $z \neq 0$,

$$
f(x, y / z, z)=f_{+}(x, y, z)+(y / z) f_{-}(x, y, y / z)
$$

for some $f_{+}$and $f_{-}$which are $\mathcal{F}$-analytic at the origin.

Proof. Write $f(x, y, z)=\sum_{i, j=0}^{\infty} f_{i, j}(x) y^{i} z^{j}$. Note that $H(x, y, z):=\sum_{i \leq j} f_{i, j}(x) y^{i} z^{j}$ is the Hadamard product in $(y, z)$ of $f(x, y, z)$ with

$$
D(x, y, z):=\frac{1}{(1-z)(1-y z)}=\sum_{i \leq j} y^{i} z^{j} .
$$

Then $f_{+}(x, y, z)=H(x, y / z, z)$, and by Cauchy's integral formula,

$$
H(x, y / z, z)=\frac{1}{2 \pi i} \int_{C} \frac{z H(x, w, z)}{z w-y} d w
$$

for some contour $C$ which is a square centered at the origin. Note that $z w-y$ is a unit on $C$ since $y / z$ may be made sufficiently small. Hence $f_{+}(x, y, z)$ is $\mathcal{F}$-analytic at the origin. The proof for $f_{-}(x, y, z)$ is similar. 
The significance of Proposition 9.3 is not that a natural set of assumptions has been found for $\mathcal{F}$ which ensures that Lion and Rolin's proof of their preparation theorem goes through for $\mathbb{R}_{\mathcal{F}}^{\mathbb{Q}}$. Rather, the significance lies in finding a reasonable answer to the following question, loosely stated:

What must we assume about $\mathcal{F}$ to ensure that the globally subanalytic integration theorem proved in 14 goes through for $\mathbb{R}_{\mathcal{F}}$ ?

Lion and Rolin's proof relies on their preparation theorem for $\mathbb{R}_{\mathrm{an}}^{\mathbb{Q}}$, so we should at least assume that $\mathcal{F}$ is a Weierstrass system. Also, to be practical, further assuming closure under Weierstrass division seems to be no significant loss. Thus $\mathcal{F}$ is closed under complexification. But $\mathcal{F}$ must surely be closed under antidifferentiation if we are to have any hope of proving an integration theorem for the definable functions of $\mathbb{R}_{\mathcal{F}}$. Then Proposition 9.3 shows that under these assumptions we can perform Lion and Rolin's splitting techniques, so the arguments of [14 go through for $\mathcal{F}$ without any needed modifications.

\section{ACKNOWLEDGMENT}

I am grateful to Patrick Speissegger for his continued support and guidance, to Jean-Phillipe Rolin for his wonderful lectures at the University of Wisconsin which got me started on this project, to Chris Miller and Lou van den Dries for their email discussions concerning Proposition 9.1, to an anonymous referee for his or her many useful comments, and to Jungyune for her continued support and patience.

\section{REFERENCES}

1. R. Bianconi, Nondefinability results for expansions of the field of real numbers by the exponential function and by the restricted sine function., J. Symb. Logic 62 (1997), 1173-1178. MR 1617985 (99k:03034)

2. E. Bierstone and P. Milman, Semianalytic and subanalytic sets, Inst. Hautes Études Sci. Publ. Math. 67 (1988), 29-54. MR0972342 (89k:32011)

3. - Canonical desingularization in characteristic zero by blowing up the maximum strata of a local invariant, Inv. Math. 128 (1997), 207-302. MR1440306 (98e:14010)

4. _ Resolution of singularities in Denjoy-Carleman classes, Selecta Math. 10 (2004), no. 1, 1-28. MR.2061220 (2005c:14074)

5. J. Bochnak, M. Coste, and M. F. Roy, Real Algebraic Geometry, Springer-Verlag, 1998. MR 1659509 (2000a:14067)

6. C. L. Childress, Weierstrass division in quasianalytic local rings, Canad. J. Math. 28 (1976), 938-953. MR0417441 (54:5491)

7. J. Denef and L. Lipshitz, Ultraproducts and approximation in local rings. II, Math. Ann. 253 (1980), 1-28. MR0594530 (82g:13021)

8. J. Denef and L. van den Dries, p-adic and real subanalytic sets, Annals of Math. 128 (1988), 79-138. MR0951508 (89k:03034)

9. A. M. Gabrielov, Projections of semi-analytic sets, Funktsionalnyi Analiz eigo prilozheniya 2 (1968), 18-30 (Russian), English translation: Funct. Anal. and its Appl. 2 (1968), 282-291. MR0245831 (39:7137)

10. Complements of subanalytic sets and existential formulas for analytic functions, Invent. Math. 125 (1996), 1-12. MR.1389958(97h:32007)

11. H. Grauert and R. Remmert, Analytische Stellenalgebren, Springer-Verlag, 1971. MR0316742 $(47: 5290)$

12. R. C. Gunning and H. Rossi, Analytic functions of several complex variables, Prentice-Hall, Englewood Cliffs, NJ, 1965. MR.0180696 (31:4927)

13. J.-M. Lion and J.-P. Rolin, Théorème de préparation pour les fonctions logarithmicoexponentielles, Ann. Inst. Fourier (Grenoble) 47 (1997), 859-884 (French). MR1465789 (98h:32009) 
14. _ Intégration des fonctions sous-analytiques et volumes des sous-ensembles sousanalytiques, Ann. Inst. Fourier (Grenoble) 48 (1998), 755-767 (French). MR1644093 (2000i:32011)

15. L. Lipshitz and L. A. Rubel, A gap theorem for power series solutions of algebraic differential equations, Amer. J. Math. 108 (1986), no. 5, 1193-1213. MR0859776 (87m:12008)

16. K. Mahler, Lectures on Transcendental Numbers, Springer-Verlag, $1976 . \quad$ MR0491533 (58:10772)

17. C. Miller, Expansions of the real field with power functions, Ann. Pure Appl. Logic 68 (1994), 79-94. MR1278550 (95i:03081)

18. Infinite differentiability in polynomially bounded o-minimal structures, Proc. Amer. Math. Soc. 123 (1995), 2551-2555. MR1257118 (95j:03069)

19. A. Parusiński, Lipschitz stratification of subanalytic sets, Ann. ENS 27 (1994), 661-996. MR:1307677 (96g:32017)

20. A. Rambaud, Quasi-analycité, o-minimalité et élimination des quantificateurs, Ph.D. thesis, Université Paris 7 - Denis Diderot, 2005.

21. J.-P. Rolin, P. Speissegger, and A. J. Wilkie, Quasianalytic Denjoy-Carleman classes and o-minimality, J. Amer. Math. Soc. 16 (2003), no. 4, 751-777 (electronic). MR1992825 (2004g:14065)

22. L. van den Dries, On the elementary theory of restricted elementary functions, J. of Symbolic Logic 53 (1988), no. 3, 796-808. MR0960999 (89i:03074)

23. L. van den Dries, A. Macintyre, and D. Marker, The elementary theory of restricted analytic fields with exponentiation, Annals of Math. 140 (1994), 183-205. MR.1289495 (95k:12015)

24. L. van den Dries and P. Speissegger, O-minimal preparation theorems, Proceedings of the Euro-Conference in Model Theory and Applications, Ravello, Italy, May 27-June 1, 2002, 87-116. MR2159715

Department of Mathematics, University of Wisconsin-Madison, 480 Lincoln Drive, MADISON, WISCONSIN 53706-1388

E-mail address: daniel_jeffrey_miller@yahoo.com 